\title{
Groupoid C*-algebras, Conformal Measures and Phase Transitions
}

\author{
Rodrigo Souza Frausino \\ DISSERTAÇÃO APRESENTADA \\ $\mathrm{AO}$ \\ Instituto de Matemática e Estatística \\ DA \\ Universidade DE SÃo PAUlo \\ PARA \\ OBTENÇÃO DO TÍTULO \\ $\mathrm{DE}$ \\ Mestre EM CiÊNCIAS \\ Programa: Mestrado em Matemática Aplicada \\ Orientador: Prof. Dr. Rodrigo Bissacot
}

Durante o desenvolvimento deste trabalho o autor recebeu auxílio financeiro da CAPES

São Paulo, Maio de 2018 
Groupoid C*-algebras, Conformal Measures and Phase Transitions

Esta é a versão original da dissertação elaborada pelo candidato Rodrigo Souza Frausino, tal como submetida à Comissão Julgadora.

Comissão Julgadora:

- Prof. Dr. Rodrigo Bissacot - IME-USP

- Prof. Dr. Ruy Exel - UFSC

- $\operatorname{Prof}^{a}$. Dr ${ }^{a}$. Cristina Cerri - IME-USP 


\section{Resumo}

Frausino, Rodrigo Souza $C^{*}$-álgebras de Grupóides, Medidas Conformes e Transições de Fase.

O objetivo deste trabalho é o estudo do fenômeno de transição de fase no contexto de Grupóides e suas $\mathrm{C}^{*}$-álgebras. O resultado principal é devido a Klaus Thomsen em [Tho17], que explora a conexão entre medidas conformes no formalismo clássico e estados KMS do contexto quântico. A transição de fase no caso quântico é consequência desta ligação entre os dois formalismos e do fato de que no setting clássico eram conhecidos exemplos de potenciais contínuos que apresentam o fenômeno de transição de fase. O potencial utilizado é aquele introduzido por Hofbauer [Hof77], um exemplo que mostra que, diferentemente de potenciais de variação somável, potenciais apenas contínuos podem apresentar transição de fase.

Palavras-chave: Grupóide, C*-álgebra, Medidas Conformes, Transição de Fase, Estados KMS. 



\section{Abstract}

Frausino, Rodrigo Souza Groupoid C*-algebras, Conformal Measures and Phase Transitions.

The objective of this work is the study of phase transitions on the context of Groupoids and their $\mathrm{C}^{*}$-Algebras. The main result of this dissertation is due to Klaus Thomsen in [Tho17], which investigates the connection between conformal measures in the classical formalism and KMS-states in the quantum formalism. The phase transition in the quantum setting is a consequence of this connection between both formalisms and the fact that on the classical setting it was known examples of continuous potentials that show the phenomena of phase transition. The potential used was introduced by Hofbauer [Hof77], an example that shows, differently from potential of summable variations, potentials only continuous can exhibit phase transition.

Keywords: Groupoid, C*-algebras, Conformal Measures, Phase Transition, KMS States. 



\section{Contents}

1 Preliminaries $\quad 5$

1.1 Thermodynamic Formalism and Hofbauer Potential . . . . . . . . . . . . 5

1.1.1 Shift Space and the Ruelle-Perron Frobenius Operator . . . . . . . . . . . 5

1.1.2 Partitions, Entropy and Pressure: introducing equilibrium states . . . . . . 6

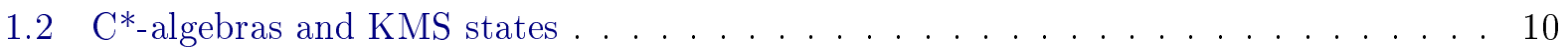

1.3 Universal $\mathrm{C}^{*}$-algebras: Generators and Relations . . . . . . . . . . . . . 17

2 Algebraic Structure $\quad 21$

2.1 Basic Definitions and results . . . . . . . . . . . . . . . . . . 21

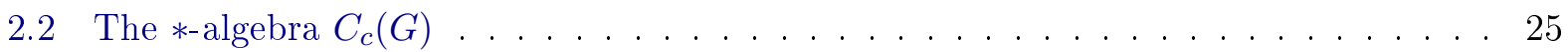

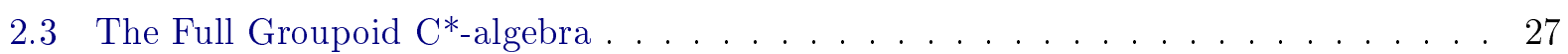

2.4 The Reduced Groupoid $\mathrm{C}^{*}$-algebra $\ldots \ldots \ldots \ldots$

3 Conformal Measures $\quad 37$

3.1 Conformal Measures . . . . . . . . . . . . . . . . . . . . . 37

3.2 The Renault-Deaconu Groupoid . . . . . . . . . . . . . . . . . . . . . . . 40

3.3 The Dinamics and KMS states . . . . . . . . . . . . . . . . 46

3.4 Cuntz-Krieger Algebras and Groupoids . . . . . . . . . . . . . . . . . . 48

4 Phase Transition and Main Result

$\begin{array}{ll}\text { Bibliography } & 61\end{array}$ 



\section{Introduction}

The phenomenon of phase transition is perhaps the most important topic in equilibrium statistical mechanics and, the mathematics involved studying models, the presence or not of phase transitions, critical temperatures, and other properties requires a high sophistication which culminated to the Fields Medal to Stanislav Smirnov in 2010.

Perhaps the most straightforward examples are the magnets, which lose their magnetic properties (attraction and repulsion of other materials) when you put them in a high temperatures. For ferromagnetic systems there exists precisely one temperature where the system changes the behavior and in this case, we call this value of critical temperature. The model proposed by the physicists to study magnets is called Ising model and is perhaps the most successfully understood of the entire statistical mechanics [FV17, Bov06, Geo11]. This model belongs to a class of models called spin systems, where the position of the particles are the vertices of a graph (for instance, $\mathbb{Z}, \mathbb{Z}^{d}$ ) and each particle has a spin associated to it. This spin usually is represented by an integer number and in many cases can assume an only finite number of values. To explore the connection with symbolic dynamics, we denote by $\mathcal{A}$ the set of spin values and we call this set of alphabet. For our propose, it will be a finite subset of $\mathbb{N}$. A unidimensional spin system is basically defined by his configuration space $\Sigma=\mathcal{A}^{\mathbb{Z}}$ and an interaction $\Phi=\left(\Phi_{\Lambda}\right)_{\Lambda}$ : a collection of continuous functions $\Phi_{\Lambda}$ which are functions depending on a finite region $\Lambda$ of the lattice $\mathbb{Z}$. The interaction defines how these spins will interact with each other and with them we can construct the measures (equilibrium, DLR, conformal etc) which describe the system.

This approach to constructing the objects with local functions is very common in the literature written by physicists, on another hand, the mathematical physics community has a big influence from ergodic theory and convex analysis, perhaps the main responsible are Y. Sinai and D. Ruelle which had a big influence on both communities. From the ergodic perspective much of this is written in terms of a function which we call potential given by $f_{\Phi}: \Sigma \rightarrow \mathbb{R}$ defined as $f_{\Phi}(x)=$ $\sum_{\Lambda \ni 0} \Phi_{\Lambda}(x) /|\Lambda|$. We can study the pressure $P\left(f_{\Phi}\right)$ and the measures which describe these systems. Naturally, the abstract generalization to study pressure, equilibrium and DLR measures for a general continuous function $f$ (which we will continue calling potential) was immediately considered. The study, from this more general point of view, is today known as thermodynamic formalism and is a big branch of ergodic theory with intense and recent activity, some classical references which consider this approach are [PP90, Bow08, Rue04, Sim14, Isr15]. For a recent review which deals with this question of the connection between potentials and interactions see [CL17].

Thanks to the famous Sinai's trick [Bow08], for suitable potentials $f: \mathcal{A}^{\mathbb{Z}} \rightarrow \mathbb{R}$, we can define a cohomologous potential $f^{\prime}: \mathcal{A}^{\mathbb{N}} \rightarrow \mathbb{R}$ such that the thermodynamics of both potentials are close and they have the same equilibrium states. After this observation, since we will stay in the unidimensional setting, we can focus our attention on potentials $f: \mathcal{A}^{\mathbb{N}} \rightarrow \mathbb{R}$ and their thermodynamic formalisms. The equilibrium measures for $f$ are shift-invariant probabilities $\mu$ which satisfy the variational problem for the pressure, that is, $P(f)=h_{\mu}+\int f d \mu$. They are one of the main objects in the classical setting. The phase transition, which physically means some change on the system, can be codified by regarding the number of equilibrium states (more generally, the DLR measures) for the potential $f$. One of the advantages of work on $\mathcal{A}^{\mathbb{N}}$ instead of $\mathcal{A}^{\mathbb{Z}}$ is the fact that we can use the machinery of the transfer (Ruelle) operator as we will see. In fact, in the case of enough regular potentials, any equilibrium measure $\mu$ comes from the Ruelle's operator. The Ruelle-PerronFrobenius theorem says that the equilibrium measures are $h d \nu$ where $h$ is an eigenfunction ( $\nu$ is an 
eigenmeasure of the dual) of the Ruelle operator from the potential $f$.

Now, remember that our concrete example were magnets who lose their magnetic properties at low temperatures, so the models should include a parameter associated with the temperature. We denote by $\beta>0$ the inverse of the temperature $T>0$ and the potential will be the function $\beta f$ and, it is usual to consider the pressure at the inverse of temperature $\beta$. In this case, we denote the pressure at temperature $\beta^{-1}$ by $P(\beta):=P(\beta f)$ and the equilibrium measures at this temperature by $\mu_{\beta}$. Sometimes we have more than one measure satisfying $P(\beta f)=h_{\mu_{\beta}}+\int \beta f d \mu_{\beta}$, this is the mathematical manifestation of the phenomenon of phase transition, for the Ising model which modelizes the magnets, this is the situation for $\beta$ large enough (low temperatures) in dimension two.

In dimension one (our setting) is more difficult to see phase transitions, if the potential is Lipschitz (or more generally, has summable variations) we have unicity of the equilibrium measure $\mu_{\beta}$ for all $\beta>0$ and the pressure function $P(\beta)$ is analytic with respect to the parameter $\beta$, see [Bow08, Rue04]. So, is natural try to find a less regular potential $f$, only continuos for example, which admits more than one equilibrium state. This was done by F. Hofbauer in [Hof77], we remember this potential at Chapter 1 .

The DLR measures (in honor of the mathematical physicists R.L. Dobrushin, O. E. Lanford and D. Ruelle) are the measures considered by the statistical mechanics community to describe the spin systems at the equilibrium; they usually call these measure by Gibbs measures. We avoid this nomenclature because there exist several measures which today are called Gibbs measures by both communities of statistical mechanics and ergodic theory. The notion of DLR measure (which we will not explain here, see the Chapter 1) was introduced in the papers [Dob68, LR69]. Roughly speaking, a DLR measure is a probability measure which is compatible with a collection of conditional probabilities with respect to sigma-algebras generated by the variables outside of finite regions $\Lambda$ of the lattice which are defined according to the interaction $\Phi$. Perhaps, at this point, the most important thing is to mention that the translation-invariant DLR measures are precisely the equilibrium measures which we just defined, see [Kel98, Rue04, Mey13].

Now, it is natural to ask what kind of condition characterizes the equilibrium states in quantum statistical mechanics, the answer is the KMS condition [HHW67], in honor of R. Kubo, P. C. Martin and J. Schwinger. The quantum analogous of the DLR states are the KMS states.

From now, we start to explore connections and analogies between the classical and quantum settings and, at this point, $\mathrm{C}^{*}$-algebras get involved. The quantum counterpart of the shift spaces $\mathcal{A}^{\mathbb{N}}$ with finite symbols are the Cuntz algebras [Cun77], denoted as $\mathcal{O}_{n}$ ( $n$ is the number of symbols), which we introduce throughout the text. We describe the KMS states in detail in Chapter 1. The motivation of K. Thomsen, and by consequence, our motivation, is to try to push the phenomenon of phase transition of the Hofbauer potential and equilibrium measures in the classical setting to $\mathrm{C}^{*}$-algebras, in this case, the Cuntz algebra $\mathcal{O}_{2}$ and the KMS states.

Connections between conformal measures (eigenmeasures from the dual of the Ruelle operator) and KMS states are already known, see, for instance, [KSS07, Ren80], so it is natural to try to push the phase transition from the classical to the quantum formalism. In fact, as we will see, the idea can be implemented and the main motivation of this thesis is to describe this path done by K. Thomsen [Tho17].

The thesis is structured in the following way:

Chapter 1: Here we set the stage. First we give a short introduction to the classical thermodynamic formalism, in particular introducing the notions of equilibrium states and phase transition; we define as well the Hofbauer potential, which is a generalization of the potential in Chapter 4 . Here we give as well an introduction to KMS states and an important way to generate $\mathrm{C}^{*}$-algebras.

Chapter 2: We define a algebraic structure known as groupoid and endow it with a compatible topology in order to study the continuous and compactly supported functions on it. We give such a space with operations such as it becomes a $*$-algebra. Finally, in this context, we are able to define two kinds of $\mathrm{C}^{*}$-algebras, the Full $\mathrm{C}^{*}$-algebra and the Reduced and we provide some properties for 
them.

Chapter 3: We define the notion of conformal measure, which plays a central role in the classical thermodynamic formalism. These measures are eigenmeasures of the Ruelle operator and, when the potential is regular enough, it is possible to show that any equilibrium measure comes from a conformal measure. To make the connection with the quantum setting, we prove that every conformal measure has a KMS state associated with it, in a proper context. We define as well the Cuntz Algebra, relating it to the algebraic structure of groupoids defined in Chapter 2.

Chapter 4: As the last chapter of this thesis, we present a result from K. Thomsen, which used a theorem from S. Neshveyev and the connection of the quantum setting with the classical one, to generate a cocycle given by a potential which is substantially the same provided by Hofbauer. The result essentially says that the phase transition in the classical sense, looking for the number of equilibrium measures, generates a phase transition concerning the number of KMS states. 



\section{Chapter 1}

\section{Preliminaries}

\subsection{Thermodynamic Formalism and Hofbauer Potential}

In this section, we present a crash course about classical thermodynamic formalism. There are a myriad of good references when we are talking about thermodynamic formalism, in particular we refer to Bowen's lecture notes [Bow08].

\subsubsection{Shift Space and the Ruelle-Perron Frobenius Operator}

Consider a finite set $\mathcal{A}$ and the countable cartesian product $\mathcal{A}^{\mathbb{N}}$. $\mathcal{A}$ is called the alphabet. One element $x \in \mathcal{A}^{\mathbb{N}}$ is a sequence, indexed by $\mathbb{N}$, of elements in $\mathcal{A}$. For all $i \in \mathbb{N}$ we denote $x_{i} \equiv \pi_{i}(x)$, $\pi_{i}$ is the canonical projection in the $i$ coordinate of $\mathcal{A}^{\mathbf{N}}$.

We wish to endow the set $\mathcal{A}^{\mathbb{N}}$ with a topology, for that, consider the metric $d: \mathcal{A}^{\mathbb{N}} \times \mathcal{A}^{\mathbb{N}} \rightarrow \mathbb{R}$, defined as

$$
d(x, y)=2^{-\inf \left\{n: x_{n} \neq y_{n}\right\}} .
$$

$\left(\mathcal{A}^{\mathbf{N}}, d\right)$ is a compact metric space. More than that, the topology induced by the metric $d$ coincides with the topology generated by cylinder sets, i.e, the sets defined for a finite word $\omega=$ $\left(\omega_{1}, \ldots, \omega_{n}\right)$ as $[\omega]:=\left\{\left(x_{i}\right)_{i \in \mathbb{N}} \in \mathcal{A}^{\mathbb{N}} \mid x_{1}=\omega_{1}, \ldots, x_{n}=\omega_{n}\right\}$. As a final topological coincidence, if we endow $\mathcal{A}$ with the discrete topology, then the product topology is equal with the previous ones. Now we can define the Shift Space with alphabet $\mathcal{A}$.

Definition 1. Given a finite set $\mathcal{A}$, the function $\sigma: \mathcal{A}^{\mathbb{N}} \rightarrow \mathcal{A}^{\mathbb{N}}$ defined by

$$
\sigma(x)_{i}=x_{i+1}, \forall x \in \mathcal{A}^{\mathbb{N}}, \forall i \in \mathbb{N}
$$

is called the shift map. The pair $\left(\mathcal{A}^{\mathbb{N}}, \sigma\right)$ is denominated as the one-sided full shift, or simply by the full shift.

From now on, let us restrain ourselves in the case $\mathcal{A}=\{1, \ldots, n\}, n \in \mathbb{N}$. In this case the full shift is denoted as $\Sigma_{n}$, i.e,

$$
\Sigma_{n}:=\{1, \ldots, n\}^{\mathbb{N}} .
$$

Now, let $A \in M_{n}(\mathbb{R})$ be a matrix with entries with only zeros or ones. We impose that $A$ is transitive, i.e, for every $i, j \in\{1,2, \ldots, n\}$ there exists an $m \in \mathbb{N}$ such that $\left(A^{m}\right)_{i j} \neq 0$. We can define the following subset of $\Sigma_{n}$, associated with $A$,

$$
\Sigma_{A}:=\left\{\left(x_{i}\right)_{i \in \mathbb{N}} \in\{1, \ldots, n\}^{\mathbb{N}} \mid A\left(x_{i}, x_{i+1}\right)=1\right\} .
$$

An important observation is that $\Sigma_{A}$ is invariant under the shift map, i.e, $\sigma\left(\Sigma_{A}\right)=\Sigma_{A}$ and it is a closed set of $\Sigma_{n}$. Now some notation for the rest of this section. Let $X$, a non empty set, and 
consider a topology on $X$ such that it is metrizable and compact. Let $T: X \rightarrow X$ a continuous function.

1. $M(X)$ is the set of probability measure over the borel $\sigma$-algebra of $X$, denoted as $\mathcal{B}_{X}$;

2. $M_{T}(X) \subset M(X)$ is the set of measures in $M(X)$ that are $T$-invariant, i.e, $\mu\left(T^{-1}(B)\right)=\mu(B)$, $\forall B \in \mathcal{B}_{X}$;

3. Given a $\sigma$-algebra $\mathcal{F}$ on $X$ and a measure $\mu$ on $\mathcal{F}$, we use a rather common notation: $\mu(f):=$ $\int_{X} f d \mu$, for all $f \mathcal{F}$-measurable;

4. $C(X)$ is the Banach space of functions of $X$ into $\mathbb{R}$, endowed with the supremum norm $\|\cdot\|_{\infty}$.

In this context, we can take $X=\Sigma_{A}$ and $T=\sigma$, the shift map. We present now the Ruelle operator, which is very important in rigorous statistical mechanics.

Definition 2. Given $\varphi \in C\left(\Sigma_{A}\right)$, the Ruelle operator in respect with $\varphi$ is the linear operator $\mathscr{L}_{\varphi}: C\left(\Sigma_{A}\right) \rightarrow C\left(\Sigma_{A}\right)$, defined as

$$
\mathscr{L}_{\varphi} f(x):=\sum_{y \in \sigma^{-1}(x)} e^{\varphi(y)} f(y), \forall x \in \Sigma_{A}
$$

Let $\varphi \in C\left(\Sigma_{A}\right)$ and define $S_{m}(\varphi(x)):=\sum_{i=0}^{m-1} \varphi\left(\sigma^{i}(x)\right)$ for all $m \in \mathbb{N}$ and for all $x \in \Sigma_{A}$.

\subsubsection{Partitions, Entropy and Pressure: introducing equilibrium states}

Before we define equilibrium states, we present the notions of partitions and relative entropy. These definitions can be found in many places, but we refer to [VO16].

Let $(X, \Sigma, \mu)$ be a probability space. Here, partition is a countable family $\mathcal{P}$ of measurable subsets of $X$, two by two disjoint and that $\mu\left(\bigcup_{P \in \mathcal{P}} P\right)=1$. Given two partitions $\mathcal{P}$ and $\mathcal{Q}$, we define $\mathcal{P} \vee \mathcal{Q}$ to be the partition whose elements are the intersections $P \cap Q$, where $P \in \mathcal{P}$ and $Q \in \mathcal{Q}$. More Generally, if $\mathcal{P}_{n}$ is a countable family of partitions, we define

$$
\bigvee_{n} \mathcal{P}_{n}:=\left\{\bigcap_{n} P_{n} \mid P_{n} \in \mathcal{P}_{n} \text { for all } \mathrm{n}\right\}
$$

The entropy of a partition $\mathcal{P}$ is the number

$$
H_{\mu}(\mathcal{P})=\sum_{P \in \mathcal{P}}-\mu(P) \log \mu(P) .
$$

Now, if $f: X \rightarrow X$ is measurable function such that $\mu\left(f^{-1}(A)\right)=\mu(A)$ for all $A \in \Sigma$, then the entropy of $f$ with respect to $\mu$ and a partition $\mathcal{P}$ is the limit

$$
h_{\mu}(f, \mathcal{P}):=\lim _{n} \frac{1}{n} H_{\mu}\left(\bigvee_{i=0}^{n-1} \mathcal{P}\right)
$$

The limit exists because one can show that the sequence $H_{\mu}\left(\bigvee_{i=0}^{n-1} \mathcal{P}\right)$ is subadditive and by Fekete's Subadditive Lemma we conclude the limit indeed exists. Finally the entropy of the system $(f, \mu)$ is defined as

$$
h_{\mu}(f)=\sup _{\mathcal{P}} h_{\mu}(f, \mathcal{P}) .
$$

Observe that we could choose $X$ to be the shift space $\Sigma_{A}$ and $\Sigma$ to be its borel $\sigma$-algebra. Next we define the pressure. 
Definition 3. The function $P: C\left(\Sigma_{A}\right) \rightarrow \mathbb{R} \cup\{+\infty\}$ defined by

$$
P(\varphi):=\lim \frac{1}{m} \log \sum_{x_{1}, \ldots, x_{m}} \sup _{y \in\left[x_{1} \ldots x_{m}\right]} e^{S_{m}(\varphi(y))},
$$

where $\left[x_{1} \ldots x_{m}\right]:=\left\{y \in \Sigma_{A}: y_{i}=x_{i}, i \in\{1, \ldots, m\}\right\}$ is the cylinder of $\Sigma_{A}$ with fixed length $m$. In these conditions $P$ is called pressure. More then that, $\mu \in M_{\sigma}\left(\Sigma_{A}\right)$ is called an equilibrium state for $\varphi$ if

$$
P(\varphi)=h_{\mu}+\mu(\varphi)
$$

in which $h_{\mu}:=h_{\mu}(\sigma)$.

Actually, the pressure can be written as

$$
P(\varphi)=\sup \left\{h_{\nu}(\sigma)+\nu(\varphi) \mid \nu \in M_{\sigma}\left(\Sigma_{A}\right)\right\} .
$$

This is the so called variational principle, Theorem 10.4.1 on [VO16]. Let $\phi \in C\left(\Sigma_{A}\right)$. We call $E_{\phi}$ to be the set of all equilibrium states for $\phi$. We prove the following lemma that it says cohomologous potentials have the same equilibrium states.

Lemma 1. Consider $\sigma: \Sigma_{A} \rightarrow \Sigma_{A}$ the shift map and $\phi, \psi \in C\left(\Sigma_{A}\right)$. If $\phi-\psi=f \circ \sigma-f+c$, for $f \in C\left(\Sigma_{A}\right)$ and $c \in \mathbb{R}$, then $E_{\phi}=E_{\psi}$.

Proof. For any $\mu \in M_{\sigma}\left(\Sigma_{A}\right)$, we have

$$
\mu(\phi-\psi)=\mu(f \circ \sigma-f+c)=\mu(f \circ \sigma)-\mu(f)+c \mu(1)=\mu(f)-\mu(f)+c=c,
$$

With some calculations,

$$
\mu(\phi)=\mu(\psi)+c \Longrightarrow h_{\mu}(\sigma)+\mu(\phi)=h_{\mu}(\sigma)+\mu(\psi)+c .
$$

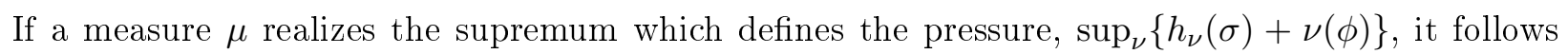
that $\mu$ will realize as well the $\operatorname{supremum}_{\sup _{\nu}}\left\{h_{\nu}(\sigma)+\mu(\psi)+c\right\}$ and $\sup _{\nu}\left\{h_{\nu}(\sigma)+\mu(\psi)\right\}$. This proves that $E_{\phi}=E_{\psi}$.

We only enunciate the next theorem, which shows how the Ruelle operator generates the equilibrium measures.

We first consider $G:=\left\{g \in C\left(\Sigma_{A}\right): g>0\right.$ and $\left.\sum_{y \in \sigma^{-1}(x)} g(y)=1, \forall x \in \Sigma_{A}\right\}$, the elements of $G$ are called $g$-functions.

If $g \in G$, clearly we have $\mathscr{L}_{\log g} f(x)=\sum_{y \in \sigma^{-1}(x)} g(y) f(y)$ and $\mathscr{L}_{\log g}[f \circ \sigma]=f$. Now we enunciate a well known Ledrappier theorem:

Theorem 1. Let $g \in G$ and $m \in M\left(\Sigma_{A}\right)$. Using the notation $\mathscr{L} \equiv \mathscr{L}_{\log g}$, it is equivalent:

1. $\mathscr{L}^{*} m=m$;

2. $m \in M_{\sigma}\left(\Sigma_{A}\right)$ and $m$ is a equilibrium state for $\log g$.

Proof. See [Wal75] page 377.

Remark 1. We call the measures satisfying the equivallent conditions of the Theorem 1 by $g$ measures. The study of $g$-measures is an active topic of research since your definition by Keane [Kea72].

Definition 4. A Borel probability measure $m$ is called a Dobrushin-Lanford-Ruelle(DLR) state for the potential $\varphi$ at inverse temperature $\beta$, if for each $n \in \mathbb{N}$

$$
\mathbb{E}\left(\mathbb{1}_{\left[x_{1}, \ldots, x_{n}\right]} \mid \sigma^{-n}(\mathcal{B})\right)(x)=\frac{1}{Z_{n}\left(\beta, x_{n+1}^{\infty}\right)} \exp \left[-\beta \sum_{i=0}^{n-1} \varphi\left(\sigma^{i}(x)\right)\right] \quad \text { for m-a.e } x \in \Sigma_{A}
$$


where $Z_{n}\left(\beta, x_{n+1}^{\infty}\right):=\sum_{y \in \sigma^{-n}\left(\sigma^{n}(x)\right)} \exp \left[-\beta \sum_{i=0}^{n-1} \varphi^{i}(y)\right]$ and $\mathcal{B}$ is the borel $\sigma$-algebra for $\Sigma_{A}$.

When considering a function $\varphi \in C\left(\Sigma_{A}\right)$ and the set of equilibrium measures $E_{\varphi}$, we might ask how many elements there are in $E_{\varphi}$. The next condition on the function $\varphi$ forces the set $E_{\varphi}$ to have only one element.

Definition 5. We say that $\varphi \in C\left(\Sigma_{A}\right)$ satisfy the Ruelle-Perron-Frobenius condition, or RPF condition, if there are $\lambda>0, h \in C\left(\Sigma_{A}\right), h>0$ and $\nu \in M\left(\Sigma_{A}\right)$ such that

1. $\mathscr{L}_{\varphi} h=\lambda h$;

2. $\mathscr{L}_{\varphi}^{*} \nu=\lambda \nu, \mathscr{L}^{*}$ is the dual of the operator $\mathscr{L}$;

3. $\nu(h)=1$;

4. $\left\|\lambda^{-m} \mathscr{L}_{\varphi}^{m} f-\nu(f) h\right\|_{\infty} \rightarrow 0$ when $m \rightarrow \infty, \forall f \in C\left(\Sigma_{A}\right)$.

The measure $\mu$ defined by

$$
\mu(f)=\nu(h f), \forall f \text { measurable }
$$

is called RPF measure.

We enunciate the next theorem, which affirms the uniqueness of the equilibrium state for a function $\phi \in C\left(\Sigma_{A}\right)$ that satisfies the RPF condition, as we said before.

Theorem 2. Let $\varphi \in C\left(\Sigma_{A}\right)$ that satisfies the RPF condition. It follows that $\mu(\cdot):=\nu(h \cdot)$ is the unique equilibrium state for $\varphi$. The measure $\nu \in M\left(\Sigma_{A}\right)$ and $h>0$ are as in the definition 5 .

Proof. See [Hof77] page 225.

Remark 2. Let $\Sigma_{A}$ a topologically mixing subshift and $\varphi: \Sigma_{A} \rightarrow \mathbb{R}$ a potential with summable variations. Then, $\varphi$ has the RPF property. For the proof, see [Bow08], page 9.

If we have a function $g: \Sigma_{n} \rightarrow \mathbb{R}$ we say it admits a Gibbs-Bowen measure $\mu \in M_{\sigma}\left(\Sigma_{n}\right)$ if the are two constants $c_{1}, c_{2}>0$ and $\lambda>0$ such that

$$
c_{1} \leq \frac{\mu\left(\left[x_{1} \cdots x_{m}\right]\right)}{\lambda^{-m} \exp \left[S_{m}(g(x))\right]} \leq c_{2}
$$

For all $x \in\left[x_{1} \cdots x_{m}\right]$ and $m \in \mathbb{N}$.

Remark 3. Let $\Sigma_{A}$ a topologically mixing subshift and $\varphi: \Sigma_{A} \rightarrow \mathbb{R}$ a potential with summable variations. By the remark $2, \varphi$ has the RPF property and then there exists a RPF measure associated to $\varphi$. This measure is Gibbs-Bowen. For the proof see [Bow08], page 15.

Remark 4. Under the same hypotheses of the remark 3, for which we know that there exists an eigenmeasure for $\varphi$, we know that the set of eigenprobabilities coincides with the set of DLR probabilities measures, see [CL17].

Next, we define the potential due to Hofbauer, which will appear again in the last chapter of this dissertation, although in a different context. This potential and its properties were the motivation behind the paper [Tho17], since the potential considered in aforementioned article is a special case when we consider the full shift with two symbols. For that define

$$
\begin{aligned}
& M_{1}:=\Sigma_{n} \backslash[1], \\
& M_{k}:=\left\{x \in \Sigma_{n}: x_{i}=1 \text { for } 1 \leq i \leq k-1 \text { and } x_{k} \neq 1\right\}, k \in\{2,3, \ldots\} .
\end{aligned}
$$

Observe that those sets are disjoint and the sequence with only ones is not in any of those sets. We see as well that $\bigcup_{k=1}^{\infty} M_{k} \cup\{(1,1,1, \ldots)\}=\Sigma_{n}$. Let $\left(a_{k}\right)_{\mathbb{N}}$ be a sequence of real numbers such that 
$\lim a_{k}=0$. Define as well the sequence $\left(s_{k}\right)_{k \in \mathbb{N}}$ as $s_{k}=\sum_{i=1}^{k} a_{k}$. The potential due to Hofbauer $g: \Sigma_{n} \rightarrow \mathbf{R}$ is given by

$$
g(x):= \begin{cases}a_{k} & \text { if } x \in M_{k} \\ 0 & \text { if } x=11 \ldots\end{cases}
$$

We observe that $g \in C\left(\Sigma_{n}\right)$. This function is interesting because when we change the behavior of the sequence $\left(a_{k}\right)$, the properties of $g$, regarding the previous definitions changes a lot. It might satisfy the RPF condition, or maybe not, but still has the uniqueness of the equilibrium states. The following table shows the many possibilities.

Table 1.1: Hofbauer Table

\begin{tabular}{|c|c|c|c|c|}
\hline \multicolumn{2}{|c|}{} & $g$ satisfies the RPF-Condition & $g$ admits a Gibbs-Bowen measure & $g$ has unique equilibrium state \\
\hline \multirow{2}{*}{$\sum e^{s_{k}}>\frac{1}{n-1}$} & $\sum a_{k}$ converges & yes & yes & yes \\
\cline { 2 - 5 } & $\sum a_{k}$ diverges & yes & no & yes \\
\hline \multirow{2}{*}{$\sum e^{s_{k}}=\frac{1}{n-1}$} & $\sum(k+1) e^{s_{k}}$ converges & no & no & yes \\
\cline { 2 - 5 } & $\sum(k+1) e^{s_{k}}$ diverges & no & no & yes \\
\hline$\sum e^{s_{k}}<\frac{1}{n-1}$ & & no & & \\
\hline
\end{tabular}

For an example, let $h$ be the potential defined in (1.8), with $a_{k}=1 / k$ and the full shift with only two letters $\Sigma_{2}$. One can see that $h$ can be written as

$$
h\left(\left(x_{i}\right)_{i=1}^{\infty}\right):= \begin{cases}\frac{1}{\min \left\{i: x_{i}=2\right\}} & \text { if } x \in M_{k} \\ 0 & \text { if } x=11 \cdots\end{cases}
$$

Indeed, if $x \in M_{k}$, then $x_{i}=1$ for $i=1, \ldots, k-1$ and $x_{k}=2$. Then $h(x)=1 / k=a_{k}$.

Generally, given a potential $f \in C\left(\Sigma_{A}\right)$, we are interested in studying the family of potentials $\{\beta f\}_{\beta>0}$ and the behavior of the set of equilibrium measures $E_{\beta f}$.

Definition 6. We say that a potential $f$ undergoes a phase transition at the inverse of temperature $\beta_{0}$, when ${ }^{1}\left|E_{\beta_{0} f}\right|>1$.

\section{The several definitions of phase transition}

One can find in the literature other possible definitions, such as: a point where the pressure is not differentiable, a point where the decay of the correlations changes etc. For some important models, all these notions coincide, for the Ising model see [ABF87]. Although, the notions using the lack of differentiability of the pressure and the number of DLR measures at certain $\beta$ do not coincide when the interaction of the model is not translation invariant, see [BCCP15].

For example, consider the potential $h$, defined in 1.9. The family $\{-\beta h\}_{\beta>0}$ have an $a_{k}(\beta)=$ $-\beta / k$. Then, $s_{k}(\beta)=-\beta \sum_{j=1}^{k} 1 / j=-\beta H_{k}$, where $H_{k}$ denote the $\mathrm{k}$-th harmonic number. One can prove that there is a $\beta_{0}$ (for the existence of $\beta_{0}$, see chapter 5 ) such that

$$
\sum_{i=1}^{\infty} \exp \left(s_{k}\left(\beta_{0}\right)\right)=1
$$

We note that the function $\beta \mapsto s_{k}(\beta)$ is decreasing, in such a way that we have following

\footnotetext{
${ }^{1}$ In Georgii [Geo11] the definition is different since the inverse of temperature $\beta$ is included in the potential $f$. He says that a potential $f$ undergoes a phase transition when $\left|E_{f}\right|>1$
} 
expressions

$$
\begin{aligned}
& \sum_{i=1}^{\infty} \exp \left(s_{k}(\beta)\right)>1 \quad \text { if } \beta<\beta_{0} \\
& \sum_{i=1}^{\infty} \exp \left(s_{k}(\beta)\right)<1 \quad \text { if } \beta>\beta_{0}
\end{aligned}
$$

By the table 1.1, in both cases we have unique equilibrium measures. In the case for $\beta_{0}$, on the other hand, we have non uniqueness. This provides us with an example of phase transition that is relevant for our purposes, for the reason that the potential we use in the quantum setting is exactly the potential $h$. In addition, in [Hof77] it is shown that the measure $\delta_{1 \infty}$ is an equilibrium measure when $\sum e^{s_{k}(\beta)}<1$ and it is interesting to see that we have a certain correspondence in the quantum seeting, since for $\beta>\beta_{0}$ we have a certain measure $m_{1 \infty}$ which creates KMS states.

\section{$1.2 \quad \mathrm{C}^{*}$-algebras and KMS states}

Since the subject of $\mathrm{C}^{*}$-algebras is really vast, in this section I have no intention of proving most of the results of $\mathrm{C}^{*}$-Algebras that is to be used in this work. A prior knowledge of operator algebras is required from the reader, standard references for this subject are [Dav96, Mur14]. This section is mostly to define the notion of KMS state and everything described here can be found in Bratteli's books [BR79, BR81]

Definition 7 . Let $(\mathfrak{A},+, \cdot)$ be an algebra over $\mathbb{C}$, we say that the operation $*: \mathfrak{A} \rightarrow \mathfrak{A}, a \mapsto a^{*}$, is an involution of the algebra $\mathfrak{A}$ if:

- $\left(a^{*}\right)^{*}=a$

- $(a b)^{*}=b^{*} a^{*}$

- $(\alpha a+\beta b)^{*}=\bar{\alpha} a^{*}+\bar{\beta} b^{*}$

for all $\alpha, \beta \in \mathbb{C}$ and for all $a, b \in \mathfrak{A}$. An algebra with such a operation is called a $*$-algebra.

The element $1 \in \mathfrak{A}$ is called the identity of the algebra if for all $a \in \mathfrak{A}$, we have $1 \cdot a=a \cdot 1$, In this case we call $\mathfrak{A}$ an algebra with identity.

Definition 8. Let $\mathfrak{A}, \mathfrak{B}$ two $*$-algebras with identity. The function $\phi: \mathfrak{A} \rightarrow \mathfrak{B}$, with properties:

- $\phi(a+\alpha b)=\phi(a)+\alpha \phi(b)$

- $\phi(a b)=\phi(a) \phi(b)$

- $\phi\left(a^{*}\right)=\phi(a)^{*}$

- $\phi\left(1_{\mathfrak{A}}\right)=1_{\mathfrak{B}}$

for all $a, b \in \mathfrak{A}$ and all $\alpha \in \mathbb{C}$ is called $a *$-homomorphism. If $\phi$ is bijective then it is called a $*$-isomorphism. If $\mathfrak{A}=\mathfrak{B}$ and $\phi$ is a $*$-isomorphism we say that $\phi$ is a $*$-automorphism.

Let $\mathfrak{A}$ be an algebra and $\|\cdot\|$ a norm in $\mathfrak{A}$ such that

$$
\|a \cdot b\| \leq\|a\|\|b\| \quad \forall a, b \in \mathfrak{A},
$$

This property is called sub-multiplicativity. We say that $(\mathfrak{A},\|\cdot\|)$ is a normed algebra. A complete(every Cauchy sequence converges) normed algebra is called a Banach algebra.

Definition 9. $\mathfrak{A}$ is a $C^{*}$-algebra if $\mathfrak{A}$ is a Banach $*$-algebra such that the following property is satisfied:

$$
\left\|a^{*} a\right\|=\|a\|^{2} \quad \forall a \in \mathfrak{U} .
$$


This property is usually called the $C^{*}$-property.

Definition 10. Let $\mathfrak{A}$ be a $C^{*}$-algebra. A linear functional $\omega$ is called a state if it is positive, i.e $\omega\left(a^{*} a\right) \geq 0$ for all $a \in \mathfrak{A}$ and it is normalized, i.e $\omega(1)=1$. Of course this only makes sense if $\mathfrak{A}$ has an identity, if it does not, we put the property that the operator norm $\|\omega\|=1$.

We say that $\tau=\left\{\tau_{t}\right\}_{t \in \mathbb{R}}$ is a 1 -parameter group of $*$-automorphisms of a $\mathrm{C}^{*}$-algebra $\mathfrak{A}$ if $\tau_{t}: \mathfrak{A} \rightarrow \mathfrak{A}$ is a $*$-automorphism in $\mathfrak{A}$ and:

i) $\tau_{t+s}=\tau_{t} \circ \tau_{s}$, for all $t, s \in \mathbb{R}$;

ii) $\tau_{0}=i d$.

Definition 11 ( $C^{*}$-Dynamical System). A $C^{*}$-dynamical system is a pair $(\mathfrak{A}, \tau)$ where $\mathfrak{A}$ is a $C^{*}$-algebra and $\tau=\left\{\tau_{t}\right\}_{t \in \mathbb{R}}$ is a 1 -parameter group of $*$-automorphisms strongly continuous, i.e $t \mapsto \tau_{t}(A)$ is continuous in the norm for all $A \in \mathfrak{A}$.

Let $X$ be a complex Banach space and $X^{*}$ its dual. Let $\sigma\left(X, X^{*}\right)$ denote the topology in $X$ induced by the functionals in $F$, i.e the weak topology on $X$.

Definition 12. A 1-parameter $t \mapsto \tau_{t}$ family of linear and bounded applications from $X$ into itself is called a one-parameter $\sigma\left(X, X^{*}\right)$-continuous group of isometries if:

1) $\tau_{t+s}=\tau_{t} \circ \tau_{s}$ for all $t, s \in \mathbb{R}$ and $\tau_{0}=i d$;

2) $\left\|\tau_{t}\right\|=1$, for all $t \in \mathbb{R}$;

3) $t \mapsto \tau_{t}(A)$ is $\sigma\left(X, X^{*}\right)$-continuous for all $A \in X$;

Definition 13 (Analytic Elements). Let $\tau$ be a one-parameter $\sigma\left(X, X^{*}\right)$-continuous group of isometries. An element $A \in X$ is analytic for $\tau$ if there exists $\lambda>0$ and a function $f: I_{\lambda} \rightarrow X$, where $I_{\lambda}=\{z \in \mathbb{C} \mid \operatorname{Im} z<\lambda\}$, such that

(i) $f(t)=\tau_{t}(A) \quad \forall t \in \mathbb{R}$;

(ii) $z \mapsto \eta(f(z))$ is analytic in the strip $I_{\lambda}$ for all $\eta \in X^{*}$.

In those conditions we write

$$
\tau_{z}(A):=f(z), \quad z \in I_{\lambda}
$$

If $\lambda=\infty$ we say that $A$ is entire analytic for $\tau$.

Proposition 1. If $t \mapsto \tau_{t}$ is a one-parameter group $\sigma\left(X, X^{*}\right)$-continuous of isometries, and $A \in X$, define

$$
A_{n}=\sqrt{\frac{n}{\pi}} \int \tau_{t}(A) e^{-n t^{2}} d t, \quad n=1,2, \cdots .
$$

Then each $A_{n}$ is an entire analytic element for $\tau,\left\|A_{n}\right\| \leq\|A\|$ for all $n$, and $A_{n} \rightarrow A$ on the $\sigma\left(X, X^{*}\right)$ topology when $n \rightarrow \infty$. In particular, the set of entire analytic elements, denoted by $X_{\tau}$, are $\sigma\left(X, X^{*}\right)$-dense in $X$.

Proof. First, define

$$
f_{n}(z):=\sqrt{\frac{n}{\pi}} \int \tau_{t}(A) e^{-n(t-z)^{2}} \mathrm{~d} t
$$

for $z \in \mathbb{C}$. It is well defined since $e^{-n(t-z)^{2}}$ is an integrable function. Note, that for $z=s \in \mathbb{R}$, 


$$
\begin{aligned}
f_{n}(s) & =\sqrt{\frac{n}{\pi}} \int \tau_{t}(A) e^{-n(t-s)^{2}} \mathrm{~d} t \\
& =\sqrt{\frac{n}{\pi}} \int \tau_{t+s}(A) e^{-n t^{2}} \mathrm{~d} t \\
& =\tau_{s}\left(\sqrt{\frac{n}{\pi}} \int \tau_{t}(A) e^{-n t^{2}} \mathrm{~d} t\right) \\
& =\tau_{s}\left(A_{n}\right) .
\end{aligned}
$$

Suppose that $\eta \in X^{*}$. We can use the inequality $\left|\eta\left(\tau_{t}(A)\right)\right| \leq\|\eta\|\|A\|$ and conclude that

$$
\begin{aligned}
& \left|\frac{\eta\left(f_{n}(z)\right)-\eta\left(f_{n}\left(z_{0}\right)\right)}{z-z_{0}}-\sqrt{\frac{n}{\pi}} \int 2 n(t-z) e^{-n(t-z)^{2}} \eta\left(\tau_{t}(A)\right) d t\right| \\
& \quad=\sqrt{\frac{n}{\pi}}\left|\int\left(\frac{e^{-n(t-z)^{2}}-e^{-n\left(t-z_{0}\right)^{2}}}{z-z_{0}}-2 n(t-z) e^{-n(t-z)^{2}}\right) \eta\left(\tau_{t}(A)\right) d t\right| \\
& \leq\|\eta\|\|A\| \sqrt{\frac{n}{\pi}} \int\left|\frac{e^{-n(t-z)^{2}}-e^{-n\left(t-z_{0}\right)^{2}}}{z-z_{0}}-2 n(t-z) e^{-n(t-z)^{2}}\right| d t .
\end{aligned}
$$

The integral on the right-hand side goes to zero when $z \rightarrow z_{0}$ and the entire analyticity follows.

In addition, we have the inequality

$$
\left\|A_{n}\right\| \leq \sup _{t}\left\|\tau_{t}(A)\right\| \sqrt{\frac{n}{\pi}} \int e^{-n t^{2}} d t=\|A\|
$$

Observe that

$$
\eta\left(A_{n}-A\right)=\eta\left(A_{n}\right)-\eta(A)=\sqrt{\frac{n}{\pi}} \int e^{-n t^{2}}\left(\eta\left(\tau_{t}(A)\right)-\eta(A)\right) \mathrm{d} t
$$

for all $\eta \in X^{*}$. On the other hand, for all $\varepsilon>0$ there is a $\delta>0$ such that $|t|<\delta$ implies that $\left|\eta\left(\tau_{t}(A)\right)-\eta(A)\right|<\frac{\varepsilon}{2}$. More than that, we can choose a $N$ such that for all $n>N$

$$
\sqrt{\frac{n}{\pi}} \int_{|t| \geq \delta} e^{-n t^{2}} \mathrm{~d} t<\frac{\varepsilon}{4\|\eta\|\|A\|}
$$

It follows that for $n>N$

$$
\begin{aligned}
\left|\eta\left(A_{n}-A\right)\right| & =\left|\eta\left(\sqrt{\frac{n}{\pi}} \int e^{-n t^{2}} \tau_{t}(A) d t-\sqrt{\frac{n}{\pi}} \int e^{-n t^{2}} A d t\right)\right| \\
= & \left|\sqrt{\frac{n}{\pi}} \int_{|t| \geq \delta} e^{-n t^{2}} \eta\left(\tau_{t}(A)-A\right) d t\right| \\
& \quad+\left|\sqrt{\frac{n}{\pi}} \int_{|t|<\delta} e^{-n t^{2}} \eta\left(\tau_{t}(A)-A\right) d t\right| \\
\leq & \sqrt{\frac{n}{\pi}} \int_{|t| \geq \delta} e^{-n t^{2}}\|\varphi\|\left(\left\|\alpha_{t}(A)\right\|+\left\|\alpha_{0}(A)\right\|\right) d t \\
& +\sqrt{\frac{n}{\pi}} \int_{|t|<\delta} e^{-n t^{2}}\left\|\varphi\left(\alpha_{t}(A)-A\right)\right\| d t \\
<\varepsilon . &
\end{aligned}
$$

Which proves that $A_{n} \rightarrow A$ in the topology $\sigma\left(X, X^{*}\right)$. 
A complex function such that condition (ii) in Definition 13 is satisfied is said to be weakanalytic. We now show that it is equivalent to strong analicity, i.e, for an interior point $z$ of the domain of $f$ the limit $\lim _{h \rightarrow 0} \frac{f(z+h)-f(z)}{h}$ exists in norm.

Proposition 2. If $A$ is $\tau_{t}$ analytic on the strip $I_{\lambda}$, then $A$ is strongly analytic on $I_{\lambda}$, i.e $f(z)=\tau_{z}(A)$ is strong analytic in the sense we explained above.

Proof. $(\Rightarrow)$ Let $\eta \in X^{*}$. For every $z \in I_{\lambda}$ there exists $r>0$ such that $D\left(z, \frac{r}{2}\right) \subset \overline{D(z, r)} \subset I_{\lambda}$. Now, for every element $z, w \in D\left(0, \frac{r}{2}\right)$, the Cauchy Integral formula for the circle $C=\{y \in \mathbb{C}|| y-z \mid=r\}$ gives us that

$$
\begin{aligned}
\left|\eta\left(\frac{f(z+h)-f(z)}{h}-\frac{f(z+w)-f(z)}{w}\right)\right| & =\frac{1}{2 \pi}\left|\int_{C} \frac{(h-w) \eta(f(y))}{(y-z)(y-z-h)(y-z-w)} d y\right| \\
& \leq \frac{2|h-w|}{9 \pi r^{2}} \sup _{y \in C}|\eta(f(y))| \\
& \leq \frac{K|h-w|}{r^{2}}\|\eta\| \sup _{y \in C}\|f(y)\|
\end{aligned}
$$

Where $K$ is just a constant. Taking the supremum over all $\eta \in X^{*}$ such that $\|\phi\|=1$ and using the fact that $\|x\|=\sup _{\|\eta\|=1}\|\eta(x)\|$ in a Banach space, we obtain

$$
\left\|\frac{f(z+h)-f(z)}{h}-\frac{f(z+w)-f(z)}{w}\right\| \leq \frac{K|z-w|}{r^{2}} \sup _{y \in C}\|f(y)\|
$$

We conclude, with the inequality above and completeness of the vector space, that the limit

$$
\lim _{h \rightarrow 0} \frac{f(z+h)-f(z)}{h}
$$

exists.

$(\Leftarrow)$ By hypothesis, for $z \in I_{\lambda}$ the limit there is $x \in X$ such that

$$
\lim _{h \rightarrow 0} \frac{f(z+h)-f(z)}{h}=x .
$$

For any $\eta \in X^{*}$,

$$
\begin{aligned}
\eta(x) & =\eta\left(\lim _{h \rightarrow 0} \frac{f(z+h)-f(z)}{h}\right) \\
& =\lim _{h \rightarrow 0} \eta\left(\frac{f(z+h)-f(z)}{h}\right) \\
& =\lim _{h \rightarrow 0} \frac{\eta(f(z+h))-\eta(f(z))}{h}
\end{aligned}
$$

This shows that $z \mapsto \eta(f(z))$ is analytic for every $z \in I_{\lambda}$

Corollary 1. If $t \mapsto \tau_{t}$ is a one parameter group $\sigma\left(X, X^{*}\right)$-continuous of isometries, then $t \mapsto \tau_{t}$ is strongly continuous and $X$ has a norm-dense set composed of entire analytic elements for $\tau$.

Proof. By Proposition 1 the set of entire elements for $\tau$ forms a $\sigma\left(X, X^{*}\right)$-dense subset of $X$. This set is a subspace, because $\tau_{t}$ are linear operators.

To show that it must be norm dense in $X$, suppose by contradiction that it is not. So let $H$ be the norm closure of the set of analytic elements for $\tau$. $H$ is a proper subspace of $X$ and suppose 
that $y \in X$ is such that $y \in X \backslash H$. By the geometric form of Hahn-Banach for the sets $H$ and $\{y\}$ there exists a $\varphi \in X^{*}$ such that

$$
\operatorname{Re} \varphi(y)<\operatorname{Re} \varphi(w), \quad w \in H .
$$

Since $H$ is a proper subspace of $X$ and $\operatorname{Re} \varphi$ is a real linear functional, $\operatorname{Re} \varphi(H)$ must be $\{0\}$ or $\mathbb{R}$. By equation (1.15), $\operatorname{Re} \varphi(H)=\{0\}$. Next, it is not difficult to prove that $\operatorname{Im} \varphi(x)=-\operatorname{Re} \varphi(i x)$, so $\operatorname{Im} \varphi(H)=\{0\}$ as well. We conclude that $\varphi$ vanishes on $H$ and $\varphi(y) \neq 0$. This contradicts the fact that for $y \in X$ there exists a sequence $\left(x_{n}\right)_{n \in \mathbb{N}}$ in $H$ that $\varphi\left(x_{n}\right) \rightarrow \varphi(x)$. Now for $A$ an analytic element, $t \mapsto \tau_{t}(A)$ is norm differentiable by Proposition 2 and hence $t \mapsto \tau_{t}(A)$ is norm continuous. For a general $A \in X$, we can find a sequence of analytic elements $A_{n}$ converging to $A$ and estimate

$$
\begin{aligned}
\left\|\tau_{t}(A)-A\right\| & \leq\left\|\tau_{t}\left(A-A_{n}\right)\right\|+\left\|\tau_{t}\left(A_{n}\right)-A_{n}\right\|+\left\|A_{n}-A\right\| \\
& =2\left\|A_{n}-A\right\|+\left\|\tau_{t}\left(A_{n}\right)-A_{n}\right\|
\end{aligned}
$$

We conclude that $\tau$ is strongly continuous.

The previous Corollary is relevant, because we can apply it for a $\mathrm{C}^{*}$-Dynamical system $(\mathfrak{A}, \tau)$, since automatically $*$-automorphisms of $\mathrm{C}^{*}$-algebras are isometries. We can finally define what is a KMS state.

Definition 14. Let $(\mathfrak{A}, \tau)$ be a $C^{*}-$ Dynamical System, $\omega$ a state in $\mathfrak{A}$ and $\beta \in \mathbb{R}$. We say $\omega$ is a $(\tau, \beta)-K M S$ state if

$$
\omega\left(A \tau_{i \beta}(B)\right)=\omega(B A)
$$

for all $A, B$ in a $*$-subalgebra $\mathfrak{A}_{0}$ composed of entire analytic elements such that $\mathfrak{A}_{0}$ is norm-dense and $\tau$-invariant (this means $\tau_{t}(A) \in \mathfrak{A}_{0}$ for all $A \in \mathfrak{A}_{0}$ and $t \in \mathbb{R}$ ).

Some simple results are summarized in the following proposition.

Proposition 3. Let $(\mathfrak{A}, \tau)$ be a $C^{*}$-Dynamical System, and $\mathfrak{A}$ is unital. Then we have the following

i) $\omega$ is a $(\tau, 0)-K M S$ state $\Leftrightarrow \omega$ is a tracial ${ }^{2}$ state.

ii) $\omega$ is a $(1, \beta)-K M S$ state $\Leftrightarrow \omega$ is a tracial state.

iii) $\omega$ is $a\left(\tau_{t}, \beta\right)-K M S$ state $\Leftrightarrow \omega$ is $\left(\tau_{t / \lambda}, \lambda \beta\right)-K M S$.

Proof. Itens i) and ii) are trivial. Let $a, b \in \mathfrak{A}_{0}$, where $\mathfrak{A}_{0}$ is the dense $*$-sub-algebra of $\mathfrak{A}$ in definition 14. We define a new 1-parameter group, $\bar{\tau}$, as $\bar{\tau}_{t}=\tau_{t / \lambda}$. By equation (14) we have,

$$
\omega\left(a \bar{\tau}_{i \lambda \beta}(b)\right)=\omega(b a) .
$$

Therefore saying that $\omega$ is a $\left(\bar{\tau}_{t}, \lambda \beta\right)-K M S$ state is the same as saying that $\omega$ is a $\left(\tau_{t / \lambda}, \lambda \beta\right)-K M S$ state.

Remark 5. Note that

$$
\tau_{a+i b}(A)=\tau_{a} \circ \tau_{i b}(A), \quad \text { para todo } A \in \mathfrak{U}
$$

where $a, b \in \mathbb{R}$. To prove that, let $A \in \mathfrak{A}$, by the Corollary 1 there is a sequence $\left\{A_{n}\right\}_{n \geq 1}$ of analytic elements such that $A_{n} \rightarrow A$. To show (1.17) it is enough to prove $\tau_{-a} \circ \tau_{a+i b}\left(A_{n}\right)=\tau_{i b}\left(A_{n}\right)$ and use the fact that $A_{n} \rightarrow A$. Using Proposition 1 we deduce

$$
\tau_{-a} \circ \tau_{a+i b}\left(A_{n}\right)=\tau_{-a}\left(\sqrt{\frac{n}{\pi}} \int \tau_{t}\left(A_{n}\right) e^{-n(t-a-i b)^{2}} d t\right)
$$

\footnotetext{
${ }^{2}$ A state $\omega$ is tracial if $\omega(A B)=\omega(B A)$
} 


$$
\begin{aligned}
& =\sqrt{\frac{n}{\pi}} \int \tau_{t-a}\left(A_{n}\right) e^{-n(t-a-i b)^{2}} d t \\
& =\sqrt{\frac{n}{\pi}} \int \tau_{t}\left(A_{n}\right) e^{-n(t-i b)^{2}} d t \\
& =\tau_{i b}\left(A_{n}\right) .
\end{aligned}
$$

We will use the following lemma from complex analysis in the proof of Theorem 3.

Lemma 2. Let $\mathcal{O} \subseteq \mathbb{C}$ be a open and connected set such that $\mathcal{V}:=\mathcal{O} \cap \mathbb{R} \neq \emptyset$. Define

$$
\mathfrak{D}=\{z \in \mathbb{C} \mid \operatorname{Im} z>0\} \cap \mathcal{O} .
$$

Let $F$ be a complex function which is holomorphic on $\mathfrak{D}$ and continuous in $\mathfrak{D} \cup \mathcal{V}$. Suppose that $F(x)=0$ for all $x \in \mathcal{V}$. Then $F(z)=0$ for all $z \in \mathfrak{D}$.

Theorem 3. Let $(\mathfrak{A}, \tau)$ be a $C^{*}$-dynamical system, $\beta \in \mathbb{R}$, and $\omega$ a state over $\mathfrak{A}$. Define a strip as,

$$
\begin{array}{ll}
\mathfrak{D}_{\beta}=\{z \in \mathbb{C} \mid 0<\operatorname{Im} z<\beta\} & \text { if } \beta>0 \\
\mathfrak{D}_{\beta}=\{z \in \mathbb{C}, \mid \beta<\operatorname{Im} z<0\} & \text { if } \beta<0
\end{array}
$$

In case $\beta=0, \mathfrak{D}_{\beta}=\mathbb{R}$. The following statements are equivalent

1. $\omega$ is a $(\tau, \beta)-K M S$ state.

2. For any $A, B \in \mathfrak{A}$ there exists a complex function $F_{A, B}$ which is analytic in the strip $\mathfrak{D}_{\beta}$ and continuous and bounded on $\overline{\mathfrak{D}_{\beta}}$ satisfying

$$
\begin{aligned}
F_{A, B}(t) & =\omega\left(A \tau_{t}(B)\right) \forall t \in \mathbb{R}, \\
F_{A, B}(t+i \beta) & =\omega\left(\tau_{t}(B) A\right) \forall t \in \mathbb{R} ;
\end{aligned}
$$

3. For any $A, B \in \mathfrak{A}$ there exists a complex function $F_{A, B}$ which is analytic in $\mathfrak{D}_{\beta}$ and continuous on $\overline{\mathfrak{D}_{\beta}}$ satisfying

$$
\begin{aligned}
F_{A, B}(t) & =\omega\left(A \tau_{t}(B)\right) \forall t \in \mathbb{R}, \\
F_{A, B}(t+i \beta) & =\omega\left(\tau_{t}(B) A\right) \forall t \in \mathbb{R} ;
\end{aligned}
$$

Proof. (1) $\Rightarrow(2)$ Let $\mathfrak{A}_{0}$ be the $*$-sub-algebra in the definition of KMS state. For $A, B$ entire analytic elements. Since $B$ is analytic, the function $t \mapsto \omega\left(A \tau_{t}(B)\right)$ has an analytic extension and we define $F_{A, B}$ as

$$
F_{A, B}(z)=\omega\left(A \tau_{z}(B)\right),
$$

for all $z \in \mathbb{C}$. Then $F_{A, B}$ is entire analytic. Since $z \mapsto \tau_{z}(A)$ is analytic, we know it must be bounded in the compact set $\{z \in \mathbb{C} \mid \operatorname{Re} z=0,0 \leq \operatorname{Im} \leq \beta\}$ and $F_{A, B}$ must be continuous in $\overline{\mathfrak{D}_{\beta}}$ as well, since

$$
\left|F_{A, B}(t+i y)\right|=\left|\omega\left(B \tau_{t}\left(\tau_{i y}(A)\right)\right)\right| \leq\|B\|\left\|\tau_{i y}(A)\right\| \leq\|B\| \sup _{0 \leq y \leq \beta}\left\|\tau_{i y}(A)\right\| .
$$

for $t+i y \in \overline{\mathfrak{D}_{\beta}}$. Observe as well that

$$
F_{A, B}(t+i \beta)=\omega\left(A \tau_{t+i \beta}(B)\right)=\omega\left(A \tau_{i \beta}\left(\tau_{t}(B)\right)\right)=\omega\left(\tau_{t}(B) A\right) .
$$

Then item (2) is satisfied when $A, B \in \mathfrak{A}_{0}$.

For $A, B \in \mathfrak{A}$ there exists sequences $A_{n}, B_{n}$, both in $\mathfrak{A}_{0}$ such that $A_{n} \rightarrow A, B_{n} \rightarrow B$. We define $F_{A_{n}, B_{n}}(z)$ as

$$
F_{A_{n}, B_{n}}(z)=\omega\left(A_{n} \tau_{z}\left(B_{n}\right)\right), \quad z \in \mathbb{C}
$$


$F_{A_{n}, B_{n}}$ are all entire function, continuous $\mathfrak{D}_{\beta}$. By the Maximum Modulus Principle it must assume its maximum at the boundary of $\mathfrak{D}_{\beta}$, so

$$
\begin{aligned}
\sup _{z \in \overline{\mathfrak{D}}_{\beta}}\left|F_{A_{n}, B_{n}}(z)\right| & =\max \left\{\sup _{t \in \mathbb{R}}\left|F_{A_{n}, B_{n}}(t)\right|, \sup _{t \in \mathbb{R}}\left|F_{A_{n}, B_{n}}(t+i \beta)\right|\right\} \\
& \leq \max \left\{\left\|A_{n} \tau_{t}\left(B_{n}\right)\right\|,\left\|\tau_{t}\left(B_{n}\right) A_{n}\right\|\right\} \\
& =\left\|A_{n}\right\|\left\|B_{n}\right\| .
\end{aligned}
$$

Let $z \in \overline{\mathfrak{D}}_{\beta}$, we have

$$
\begin{aligned}
F_{A_{n}, B_{n}}(z)-F_{A_{m}, B_{m}}(z) & =\omega\left(A_{n} \tau_{z}\left(B_{n}\right)\right)-\omega\left(A_{m} \tau_{z}\left(B_{m}\right)\right) \\
& =\omega\left(\left(A_{n}-A_{m}\right) \tau_{z}\left(B_{n}\right)\right)+\omega\left(\left(A_{m}\right)\left(\tau_{z}\left(B_{n}\right)-\tau_{z}\left(B_{m}\right)\right)\right) \\
& =F_{A_{n}-A_{m}, B_{n}}(z)+F_{A_{m}, B_{n}-B_{m}}(z),
\end{aligned}
$$

hence

$$
\left|F_{A_{n}, B_{n}}(z)-F_{A_{m}, B_{m}}(z)\right| \leq\left\|A_{n}-A_{m}\right\|\left\|B_{n}\right\|+\left\|A_{m}\right\|\left\|B_{n}-B_{m}\right\|,
$$

for all $z \in \overline{\mathfrak{D}}_{\beta}$.

Therefore, the sequence $\left\{F_{A_{n}, B_{n}}(z)\right\}_{n \geq 1}$ is a uniform Cauchy sequence. We can define $F_{A, B}(z)=$ $\lim _{n} F_{A_{n}, B_{n}}$. As the convergence is uniform, $F_{A, B}$ is an analytic function on $\mathfrak{D}_{\beta}$, continuous and bounded at $\overline{\mathfrak{D}}_{\beta}$. More than that,

$$
\begin{aligned}
F_{A, B}(t) & =\lim _{n \rightarrow \infty} F_{A_{n}, B_{n}}(t)=\lim _{n \rightarrow \infty} \omega\left(A_{n} \tau_{t}\left(B_{n}\right)\right)=\omega\left(A \tau_{t}(B)\right) \\
F_{A, B}(t+i \beta) & =\lim _{n \rightarrow \infty} F_{A_{n}, B_{n}}(t+i \beta)=\lim _{n \rightarrow \infty} \omega\left(\tau_{t}\left(B_{n}\right) A_{n}\right)=\omega\left(\tau_{t}(B) A\right) .
\end{aligned}
$$

Proving item (2).

$(2) \Rightarrow(3)$ Trivial.

$(3) \Rightarrow(1)$ If $A, B \in \mathfrak{A}_{0}$, define

$$
G_{A, B}(z)=\omega\left(A \tau_{z}(B)\right)
$$

for all $z \in \mathbb{C}$. Then $G_{A, B}$ is entire analytic and

$$
G_{A, B}(t)=\omega\left(A \tau_{t}(B)\right)=F_{A, B}(t),
$$

for all $t \in \mathbb{R}$. Define $h(z)=G_{A, B}(z)-F_{A, B}(z)$ and choose $\mathcal{O}=\mathbb{R} \times(-\beta, \beta)$. We have that $h$ satisfies the hypothesis Lemma 2 , so $h(z)=0$ for all $z \in \mathbb{R} \times[0, \beta)$, we have

$$
G_{A, B}(z)=F_{A, B}(z)=\omega\left(A \tau_{z}(B)\right), \forall z \in \mathbb{R} \times[0, \beta),
$$

but

$$
F_{A, B}(t+i \beta)=\omega\left(A \tau_{t+i \beta}(B)\right)=G_{A, B}(t+i \beta) .
$$

Hence,

$$
F_{A, B}(z)=\omega\left(A \tau_{z}(B)\right), \quad \forall z \in \overline{\mathfrak{D}}_{\beta} .
$$

We conclude that $\omega\left(A \tau_{i \beta}(B)\right)=F_{A, B}(i \beta)=\omega(B A), \quad \forall A, B \in \mathfrak{A}_{0}$. It is then proved that $\omega$ is a $(\tau, \beta)-\mathrm{KMS}$ state.

We shall see now that a KMS state is invariant under its dynamic.

Proposition 4. Let $\omega$ be an $(\tau, \beta)-K M S$ state over the $C^{*}$-algebra $\mathfrak{A}$ with $\beta \in \mathbb{R} \backslash\{0\}$. Then $\omega$ is $\tau$ invariant, i.e

$$
\omega\left(\tau_{t}(A)\right)=\omega(A)
$$

for all $A \in \mathfrak{A}$ and all $t \in \mathbb{R}$. 
Proof. We assume $\beta>0$. For $A \in \mathfrak{A}_{0}$, the $*$-sub-algebra from the definition of KMS state, we set the function $f: \mathbb{C} \rightarrow \mathbb{C}$ as

$$
f(z)=\omega\left(\tau_{z}(A)\right)
$$

Then $f$ is analytic on $\mathbb{C}$ (or entire), since $A$ is an entire analytic element for $\tau$.

Let $z \in \mathbb{R} \times[0, \beta]$, then:

$$
|f(z)|=\left|\omega\left(\tau_{z}(A)\right)\right| \leq\left\|\tau_{z}(A)\right\|=\left\|\tau_{R e} z \circ \tau_{i \operatorname{Im} z}(A)\right\| \leq\left\|\tau_{i \operatorname{Im}_{z}}(A)\right\| \leq M<\infty
$$

where $M=\sup \left\{\left\|\tau_{i t}(A)\right\| \mid t \in[0, \beta]\right\}$. It is finite because $\gamma \in[0, \beta] \rightarrow\left\|\tau_{i \gamma}(B)\right\|$ is continuous. Then we have that $f(z)$ is bounded on the strip $\mathbb{R} \times[0, \beta]$.

We show that $f(z)$ is periodic with period $i \beta$ :

$$
f(z+i \beta)=\omega\left(\tau_{z+i \beta}(A)\right)=\omega\left(1 \tau_{i \beta}\left(\tau_{z}(A)\right)\right)=\omega\left(\tau_{z}(A) 1\right)=\omega\left(\tau_{z}(A)\right)=f(z) .
$$

Above we used the KMS condition and that $\tau_{z}(A)$ is a entire analytic element for $\tau$. Then we have that $f(z)$ is bounded and analytic on $\mathbb{C}$. By Liouville's Theorem we have that $f(z)$ is constant and

$$
\omega(A)=f(0)=f(t)=\omega\left(\tau_{t}(A)\right), \text { for all } t \in \mathbb{R} .
$$

Since $\mathfrak{A}_{0}$ is dense on $\mathfrak{A}$ is follows that $\omega$ is $\tau$-invariant

Now the following proposition will show us that the set of $\beta$ such that there is a $(\tau, \beta)-K M S$ state, is closed.

Proposition 5. Let $(\mathfrak{A}, \tau)$ a $C^{*}$-dynamical system and $\omega_{\beta_{n}}\left(\tau, \beta_{n}\right)-K M S$ for $(\mathfrak{A}, \tau)$ and $\beta_{n} \rightarrow \beta$. Then any accumulation point $\omega$ of $\left\{\omega_{\beta_{n}}\right\}$ is a $(\tau, \beta)-K M S$ state for $(\mathfrak{A}, \tau)$.

Proof. Let $\omega$ be an accumulation point of $\left\{\omega_{\beta_{n}}\right\}$. Without loss of generality, we assume that $\omega_{\beta_{n}} \rightarrow$ $\omega$. Let $A, B$ be entire analytic elements for $\tau$, then $\tau_{i \beta_{n}} \rightarrow \tau_{i \beta}(B)$, we have

$$
\begin{aligned}
\left|\omega\left(A \tau_{i \beta}(B)\right)-\omega(B A)\right| & \leq\left|\omega\left(A \tau_{i \beta}(B)\right)-\omega_{\beta_{n}}\left(A \tau_{i \beta_{n}}(B)\right)\right| \\
& +\left|\omega_{\beta_{n}}\left(A \tau_{i \beta_{n}}(B)\right)-\omega_{\beta_{n}}(B A)\right| \\
& +\left|\omega_{\beta_{n}}(B A)-\omega(B A)\right|
\end{aligned}
$$

We just need to estimate the three terms on the right side. (1.22) goes to zero because $\omega_{\beta_{n}} \rightarrow \omega$. (1.21) is zero because of the KMS condition. The first we do a new estimation,

$$
\begin{aligned}
\left|\omega\left(A \tau_{i \beta}(B)\right)-\omega_{\beta_{n}}\left(A \tau_{i \beta_{n}}(B)\right)\right| & \leq\left|\omega\left(A \tau_{i \beta}(B)\right)-\omega_{\beta_{n}}\left(A \tau_{i \beta}(B)\right)\right| \\
& +\left|\omega_{\beta_{n}}\left(A \tau_{i \beta}(B)\right)-\omega_{\beta_{n}}\left(A \tau_{i \beta_{n}}(B)\right)\right|
\end{aligned}
$$

Now, equation (1.23) goes to zero because $\omega_{\beta_{n}} \rightarrow \omega$. Equation 1.24 goes to zero, since $\omega_{\beta_{n}} \rightarrow \omega$ and $\tau_{i \beta_{n}} \rightarrow \tau_{i \beta}$. We conclude that $\omega\left(A \tau_{i \beta}(B)\right)=\omega(B A)$, so $\omega$ is $(\tau, \beta)-\mathrm{KMS}$.

\subsection{Universal $\mathrm{C}^{*}$-algebras: Generators and Relations}

This section is based on [Bla85, Phi89]. Here we shall be observing an important way to create $\mathrm{C}^{*}$-algebras.

In this section we consider a set $G$ which we call its elements by generators. We define a set $G^{*}:=\left\{g^{*}: g \in G\right\}$. Then we denote by $\mathcal{F}(G)$ the free associative complex (or real) *-algebra (without identity) on $G$, i.e., the set of all polynomials in the noncommuting variables $G \sqcup G^{*}$ 
(disjoint union), with complex coefficients and no constant term. By construction, any function $f: G \rightarrow \mathfrak{A}$, where $\mathfrak{A}$ is a $\mathrm{C}^{*}$-algebra, extends to a unique $*$-homomorphism from $\mathcal{F}(G)$ to $\mathfrak{A}$, which we also call $f$.

We also will consider a set $R$ of relations on $G$, consisting in a collection of statements about the elements of $G$ which can be formulated for elements of a $C^{*}$-algebra. These are some examples:

- $\|x\| \leq 1$, where $x$ can an element of $S \subseteq \mathcal{F}(G)$;

- $x$ is positive, $x \in S \subseteq \mathcal{F}(G)$;

- $x=x^{*}$ for a specific $x \in G$

Since we want to see the generators as elements of a $\mathrm{C}^{*}$-algebra, we need to consider functions from $G$ to $\mathfrak{A}$, where $\mathfrak{A}$ is any $\mathrm{C}^{*}$-algebra. However we need to keep the relations in $R$ valid on $\mathfrak{A}$. That motivates the following definition.

Definition 15. Let $(G, R)$ be a pair of a set of generators and a set of relations. A representation of $(G, R)$ in a $C^{*}$-algebra $\mathfrak{A}$ is a function $\pi: G \rightarrow \mathfrak{A}$ such that the elements the relations in $R$ are satisfied in $\mathfrak{A}$ when we replace $g$ by $\pi(g)$ for all $g \in G$. A representation on a Hilbert space $\mathcal{H}$ is a representation in $\mathfrak{B}(\mathcal{H})$.

Now we will establish some conditions which will assure the well definition of a universal $\mathrm{C}^{*}$ algebra.

Definition 16 (Admissibility). A pair $(G, R)$ of generators and relations is said to be admissible if the following conditions hold:

(1) the function from $G$ to the zero $C^{*}$-algebra is a representation of $(G, R)$;

(2) if $\pi$ is a representation of $(G, R)$ in a $C^{*}$-algebra $A$ and $B$ is a $C^{*}$-subalgebra of $A$ which contains $\pi(G)$, then $\pi$ is a representation of $(G, R)$ in $B$;

(3) if $\pi$ is a representation of $(G, R)$ in a $C^{*}$-algebra $A$ and $\varphi: A \rightarrow B$ is a surjective *homomorphism between $C^{*}$-algebras, then $\varphi \circ \pi$ is a representation of $(G, R)$ in $B$;

(4) for every $g \in G$ there is a constant $M(g)$ such that $\|\pi(g)\| \leq M(g)$ for every representation of $(G, R)$ on any $C^{*}$-algebra;

(5) if $\left\{\pi_{\alpha}\right\}_{\alpha \in I}$ is a family of representations of $(G, R)$ on Hilbert spaces $H_{\alpha}$, then $g \mapsto \pi(g):=$ $\bigoplus_{\alpha \in I} \pi_{\alpha}(g)$ is a representation of $(G, R)$ on $\mathcal{H}=\bigoplus_{\alpha \in I} \mathcal{H}_{\alpha}$.

Remark 6. In presence of (3), the condition (1) is equivalent to "there exists a representation of $(G, R)$ ”.

Some pairs $(G, R)$ which are not admissible:

(a) $G=\{x\}$ and $R=\{“\|x\|=1$ " $\}$ does not satisfy (1);

(b) $G=\{1, u\}$ and $R=\left\{\right.$ " 1 is an identity ", " $u u^{*}=u^{*} u=1$ ", "there exists a continuous path $t \mapsto$ $u_{t}$ with $\left.u_{0}=1, u_{1}=u, u_{t} u_{t}^{*}=u_{t}^{*} u_{t}=1 "\right\}$ satisfy (1) but not (2);

(c) $G=\{x\}$ and $R=\{“\|x\| \in\{0,1\} "\}$ satisfy (1) and (2), but not (3);

(d) $G=\{x\}$ and $R=\left\{“ x^{*}=x "\right\}$ satisfy (1), (2) and (3), but not (4);

(e) $G=\{x\}$ and $R=\left\{" x^{*}=x ", "\|x\|<1\right.$ " $\}$ satisfy (1), (2), (3) and (4), but not (5). 
Definition 17 (Universal $C^{*}$-algebra on the Generators and Relations). Given a pair of generators and relations $(G, R)$, the universal $C^{*}$-algebra on $(G, R)$ is a $C^{*}$-algebra $C^{*}(G, R)$ with a representation $\pi: G \rightarrow C^{*}(G, R)$, such that, given any representation $\zeta: G \rightarrow B$, with $B$ being a $C^{*}$-algebra, there exists an unique $*$-homomorphism $\varphi: C^{*}(G, R) \rightarrow B$ such that $\zeta=\varphi \circ \pi$.

Notice that, by construction, any two universal $\mathrm{C}^{*}$-algebras on the same pair $(G, R)$ are isomorphic with an unique isomorphism. This property is called "universal property". And now, the most important result on this construction.

Theorem 4. If $(G, R)$ is admissible, then $C^{*}(G, R)$ exists. Moreover, $C^{*}(G, R)$ is the Hausdorff completion $^{3}$ of $\mathcal{F}(G)$ in the $C^{*}$-seminorm

$$
\|x\|:=\sup \{\|\pi(x)\|: \pi \text { is a representation of }(G, R)\} .
$$

Proof. By construction, any function from $G$ to any $\mathrm{C}^{*}$-algebra $\mathfrak{A}$ extends to an unique $*$-homomorphism from $\mathcal{F}(G)$ to $\mathfrak{A}$, and in particular this result is valid for representations of $(G, R)$ in $\mathfrak{A}$ and on Hilbert spaces. Such representations do exist because the validity of the condition (1) of the definition 16.

The condition (4) in the same definition grants that $\|x\|<\infty$ for any $x \in \mathcal{F}(G)$, then (1.25) is well defined as a $C^{*}$-seminorm and the Hausdorff completion of $\mathcal{F}(G)$ is granted, leading to a $\mathrm{C}^{*}$ algebra. The condition (5) guarantees that the obvious map $G \rightarrow C^{*}(G, R)$ is also a representation. The conditions (2) and (3) ensure the universal property.

\footnotetext{
${ }^{3}$ Quotient by the usual equivalence relation of the $C^{*}$-seminorm.
} 



\section{Chapter 2}

\section{Algebraic Structure}

In this Chapter we will define what is a Groupoid, giving some examples for a better intuition and some propositions about this structure. We have as a objective the study of the $\mathrm{C}^{*}$-algebras associated with those Groupoids. We used manly [Sim, Put].

\subsection{Basic Definitions and results}

Before we give the definition of a Groupoid, be aware that such definition varies throughout the literature and in particular the next definition is not how it is in [Put], but they are in fact equivalent.

\section{Definition 18. (Groupoid)}

A groupoid consists of a set $G$, a subset $G^{(0)} \subset G$ called units or the objects, two surjective maps $s, r: G \rightarrow G^{(0)}$ (called source and range maps) and a law of composition $\left(y_{1}, y_{2}\right) \in G^{(2)} \rightarrow y_{1} y_{2} \in G$, where

$$
G^{(2)}=\left\{\left(y_{1}, y_{2}\right) \in G \times G: s\left(y_{1}\right)=r\left(y_{2}\right)\right\}
$$

$G^{(2)}$ is called the set of composable parts. A groupoid must have the properties:

1. $s\left(y_{1} y_{2}\right)=s\left(y_{2}\right), r\left(y_{1} y_{2}\right)=r\left(y_{1}\right) \forall\left(y_{1}, y_{2}\right) \in G^{(2)}$;

2. $s(x)=r(x)=x \forall x \in G^{(0)}$;

3. $y s(y)=y, r(y) y=y \forall y \in G$;

4. $\left(y_{1} y_{2}\right) y_{3}=y_{1}\left(y_{2} y_{3}\right)$ when $\left(y_{1}, y_{2}\right) \in G^{(2)}$ and $\left(y_{2}, y_{3}\right) \in G^{(2)}$

5. each $y$ has a two-sided inverse $y^{-1}$, so that $y y^{-1}=r(y), y^{-1} y=s(y)$

The definition above does not seem natural as it is, however there is a really good picture of how a groupoid operates.

The elements of a groupoid $G$ can be thought as an arrow between two nodes, much like this

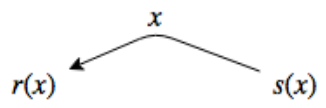

The composition/product of a groupoid can be thought as a concatenation of arrow,

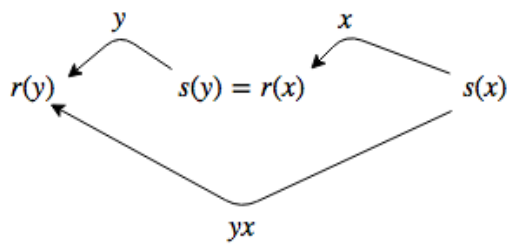


And for the inverse.

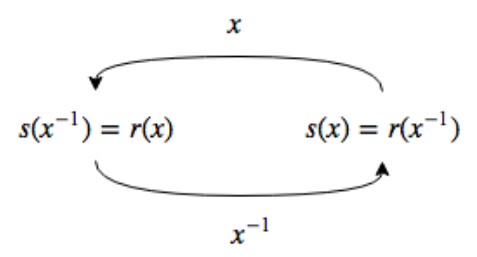

The reader is invited to see how these pictures are related with all the axioms of a groupoid. For example, axiom 1 is simply saying that the source of the concatenation of two arrows is the same as the source of the first one. We think similarly about the range of the concatenation.

Example 1. As a simple example (probably the most simple) let $H$ be a group and e its identity. Let $G=H$ and $G^{(0)}=\{e\}$. The range and source maps, both of them are constant maps $s(x)=$ $r(y)=e \quad \forall x, y \in G$. The law of composition is the same as the Group. We see that $G^{(2)}=G \times G$ and all the itens on the definition of a groupoid is easily verified.

A picture of a group $H$, as a groupoid, is of a loop.

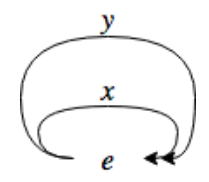

Example 2. Let $X$ be a set. We can think the cartesian product $G:=X \times X$ as groupoid such that the unit space $G^{(0)}$ can be identified with $X$. Given an element $g=(x, y) \in X \times X$ we define the functions range and source as $r(g)=(x, x)$ and $s(g)=(y, y) . G^{(0)}:=(x, x): x \in X$. Note that we can identify $G^{(0)}$ with $X$ with the canonical map $(x, x) \mapsto x$ Let $h=(w, z)$, then the $(g, h) \in G^{(2)}$ if and only if $s(g)=r(h)$, i.e $(y, y)=(w, w)$ or simply $y=w$. We define the product as $(x, y)(y, z)=(x, z)$ and the inverse as $(x, y)^{-1}=(y, x)$. It is not difficult to verify the axioms. Now, the nice picture here is that a pair $(x, y) \in G$ can be thought as an arrow from $y$ into $x$ and the mental image is the same as what we described before.

Definition 19. We say that a groupoid $G$ is a topological groupoid if it is endowed with a topology such that all structure maps are continuous, i.e the source, range, composition and inverse maps are continuous.

We note that the topology on $G^{(2)}$ is the one induced by the product topology. We don't need to check all of those maps as the next proposition tells.

Proposition 6. In a topological Groupoid $G$, continuity of the inverse map and the composition implies continuity of the source and range maps.

Proof. We show only that the range map is continuous, the proof is analogous for the source map. Denote the composition map by $c$ and the inverse map by $f$. Define $\rho: G \rightarrow G^{(2)}$ by $\rho(y)=\left(y, y^{-1}\right)$. Then the range map $r$ can be written as

$$
r=c \circ \rho .
$$

This implies that the continuity of the range map depends on the continuity of $\rho$. Let $W \subset G^{(2)}$ be an open set. Without loss of generality, $W$ has the form $\pi_{1}^{-1}(U) \cap \pi_{2}^{-1}(V) \cap G^{(2)}$, where those $\pi_{i}$ denote the projection of $G \times G$ on the i-coordinate and $U, V$ are open sets of $G$. This is true since these kind of sets form a basis for the topology of $G^{(2)}$. Let $y \in \rho^{-1}(W)$. The inverse map is continuous so $f^{-1}(V) \cap U$ is an open set that contains $y$ and it is contained in $\rho^{-1}(W)$. This proves that every $y \in \rho^{-1}(W)$ is an interior point, making $\rho^{-1}(W)$ an open set. $\rho$ is therefore continuous and we conclude the proposition. 
Definition 20. (Local Homeomorphism) A map $f: X \rightarrow Y$ between two topological spaces is a local homeomorphism if, for every $x$ in $X$, there is an open set $U$ containing $x$ such that $f(U)$ is open in $Y$ and $\left.f\right|_{U}: U \rightarrow f(U)$ is a homeomorphism.

Proposition 7. Let $X, Y$ be topological spaces and $f: X \rightarrow Y$ a local homeomorphism. Then $f$ is a continuous open map.

Proof. First we prove continuity. Let $x \in X$. We want to show that, for every open neighborhood $V$ of $f(x)$, there exists a neighborhood $U$ of $x$ such that $f(U) \subseteq V$. Let $U_{x}$ be an open neighborhood of $x$ and $V_{x}$ an open set in $Y$ such that $f$ induces a homeomorphism $\left.f\right|_{U_{x}}: U_{x} \rightarrow V_{x}$ and choose any open neighborhood $V$ of $f(x)$. Then $V \cap V_{x}$ is an open set in $Y$ containing $f(x)$, so there exists an open neighborhood $U$ of $x$ in $U_{x}$ such that $f(U) \subseteq V \cap V_{x}$; since $U$ is open in $U_{x}$ it is open in $X$ as well and $f(U) \subseteq V$ as requested.

Now we want to prove that $f$ is open. Let $A$ be open in $X$ and, for each $x \in A$, choose open sets $U_{x} \subseteq X$ and $V_{x} \subseteq Y$ so that $x \in U_{x}$ and $f$ induces a homeomorphism between $U_{x}$ and $V_{x}$. For each $x \in A, f\left(U_{x} \cap A\right)$ is open in $V_{x}$, so it is open in $Y$ as well. Therefore

$$
\bigcup_{x \in A} f\left(U_{x} \cap A\right)=f(A)
$$

and $f(A)$, as a union of open sets, must be open itself.

\section{Definition 21. (Étale Groupoid)}

A topological groupoid $G$ is said to be étale if $s$ and $r$ are local homeomorphisms. Any open set $U$ as in definition (20) is called an open bisection.

Remark 7. When $G$ is an étale groupoid, a sufficient condition to prove that an open set $W \subset G$ is also an open bisection is to prove that $r$ and $s$ are injective maps on $W$. Indeed since $r$ and $s$ are open maps $r(W)$ and $s(W)$ are open. $\left.r\right|_{W}$ is again an open map and it is continuous, the inverse is continuous as well, so $\left.r\right|_{W}$ is a homeomorphism, similarly for $s$.

In this work, we deal with groupoids whose topology is locally compact Hausdorff and étale. We won't be dealing with other types of topological groupoids.

Proposition 8. If $G$ is an étale groupoid, then the subspace topology on $G_{x}:=\{y \in G: s(y)=x\}$ and $G^{x}:=\{y \in G: r(y)=x\}$ is equivalent to the discrete topology for all $x \in G^{(0)}$.

Proof. The subspace topology is trivially contained in the discrete topology. Now assume for a contradiction that there exists some $z \in G_{x}$ such that $\{z\}$ is not open in the subspace topology. Then any open set, $U$, of $G$, which $z \in U$ must contain another element $y$ in $G_{x}$. Since $s$ is a local homeomorphism, there exists a open set $W$ of $G$, which $z \in W$ and $s$ is injective on $W$. This contradicts the previous statement, because there is no $y \in G_{x}$ such that $y \in W$. Hence we proved that $\{z\}$ is open in the subspace topology of $G_{x}$, hence this topology is the discrete one. The same argument can be done for $G^{x}$.

Proposition 9. If $G$ is a $L C H$ étale groupoid, then $G^{(0)}$ is a clopen subset of $G$. The topology on $G^{(0)}$ is the one induced by $G$.

Proof. We divide the proof in two parts.

$G^{(0)}$ is Closed:: Let $u_{i}$ be a net in $G^{(0)}$ that converges to $u \in G$. The functions $r$ and $s$ are continuous, so $r\left(u_{i}\right) \rightarrow r(u)$ and $s\left(u_{i}\right) \rightarrow s(u)$. As $u_{i} \in G^{(0)}$, we have $r\left(u_{i}\right)=s\left(u_{i}\right)=u_{i}$. Hence $u=s(u)=r(u) \in G^{(0)}$, i.e, $G^{(0)}$ is closed in $G$.

$G^{(0)}$ is Open: Let $x \in G^{(0)}$. Since $G$ is étale, there is an open $U$ containing $x$ such that $s$ is a homeomorphism on $U$. By the continuity of $s, s^{-1}(U)$ is open in $G$. Consider $V:=U \cap s^{-1}(U)$, an 
open set containing $x$. We observe that $V \subseteq G^{(0)}$, since $s(\gamma)=s(s(\gamma))$ for all $\gamma \in V$. This shows that $x$ is an interior point and $G^{(0)}$ is open.

We need some properties for the set of bisections.

Proposition 10. Let $G$ be an étale groupoid, then the set of bisections of $G$ form an open basis for the topology of $G$.

Proof. Given two open bisection $V$ and $W$, it is clear that $V \cap W$ is an open bisection as well. Now, let $U$ be an arbitrary open set of $G$, then for every $x \in U$, there exists open sets $V_{1}^{x}, V_{2}^{x}$ such that $x \in V_{1}^{x} \cap V_{2}^{x}$ and $\left.r\right|_{V_{1}^{x}}$ is injective and $\left.s\right|_{V_{2}^{x}}$ as well. If $H_{x}:=V_{1}^{x} \cap V_{2}^{x} \cap U, H_{x}$ is an open bisection and $\bigcup_{x \in U} H_{x}=U$. We conclude that the set of all bisections form an open basis for $G$.

Proposition 11. Let $G$ be an étale groupoid, then if $U, V$ are open bisections,

1. $U^{-1}:=\left\{\gamma^{-1}: \gamma \in U\right\}$ is an open bisection.

2. If $p$ is the composition/product map of $G$, then $U V:=\{\gamma \eta: \gamma \in U, \eta \in V$ and $(\gamma, \eta) \in$ $\left.G^{(2)}\right\}=p\left((U \times V) \cap G^{(2)}\right)$ is an open bisection.

Proof. Let us prove the first assertion. The inversion map $i$ is a homeomorphism, because it is bijective, continuous and the inverse is itself, i.e $i^{-1}=i$. Hence $U^{-1}$ is an open set. Now for $x, y \in U^{-1}$ such that $r(x)=r(y)$, we have that $x^{-1}, y^{-1} \in U$ and $r(x)=s\left(x^{-1}\right)=r(y)=s\left(y^{-1}\right)$. Since $U$ is an open bisection, $y^{-1}=x^{-1}$ which implies $x=y$. A similar argument shows that $s$ is injective in $U^{-1}$ and we conclude that $U^{-1}$ is an open bisection.

Now we prove the second assertion. We start by defining $W=s(U) \cap r(V)$, an open subset of $G^{(0)}$. Let $U^{\prime}=\left.s\right|_{U} ^{-1}(W) \subset U$ and $V^{\prime}=\left.r\right|_{V} ^{-1}(W) \subset V$. $U^{\prime}$ and $V^{\prime}$ are open bisections, because they are open subsets of an open bisection. We have $s\left(U^{\prime}\right)=r\left(V^{\prime}\right)=W$. In addition, $U \times V \cap G^{(2)}=U^{\prime} \times$ $V^{\prime} \cap G^{(2)}$, because if $(g, h) \in U \times V \cap G^{(2)}$, then $s(g)=r(h) \in W$, so there are $g^{\prime} \in U^{\prime}, h^{\prime} \in V^{\prime}$ such that $s(g)=s\left(g^{\prime}\right)$ and $r(h)=r\left(h^{\prime}\right)$. Nevertheless as $U^{\prime}$ and $V^{\prime}$ are subsets of $U$ and $V$ respectively, by the injective property of $r$ and $s, g=g^{\prime}$ and $h=h^{\prime}$. This justifies $U \times V \cap G^{(2)}=U^{\prime} \times V^{\prime} \cap G^{(2)}$. We can now, without loss of generality, suppose that our open bisections satisfy $s(U)=r(V)$.

$U \times V \cap G^{(2)}$ is an open set of $G^{(2)}$. The map $\phi:=\left.\left.r\right|_{V} ^{-1} \circ s\right|_{U}: U \rightarrow V$ is a homeomorphism, since it is a composition of homeomorphisms. The map $f$ from $U$ to $U \times V$ defined by $f\left(g^{\prime}\right)=\left(g^{\prime}, \phi\left(g^{\prime}\right)\right)$ has image in $G^{(2)}$, since $r\left(\phi\left(g^{\prime}\right)\right)=s\left(g^{\prime}\right)$. We claim that its image is precisely $U \times V \cap G^{(2)}$. We already know that its image is contained in $U \times V \cap G^{(2)}$. For the reverse inclusion, suppose $(g, h) \in G^{(2)}$. Then $g \in U, h \in V$ and $s(g)=r(h)$. It follows that $h=\phi(g)$ and we proved the claim. $f$ is injective. Since $\phi$ is continuous, and $f$ is basically the identity on the first coordinate, $f$ is continuous as well. Denote by $\pi_{1}$ the projection of $U \times V \cap G^{(2)}$ in $U$. Clearly $\pi_{1} \circ f$ is the identity on $U$ and using the fact that $f$ is onto $U \times V \cap G^{(2)}$, we conclude that $\pi_{1}$ is injective. In addition, for all $u \in U$, we have $s(u) \in s(U)=r(V)$, so $s(u)=r(v)$ for some $v \in V$, then $(u, v) \in U \times V \cap G^{(2)}$ and $\pi_{1}((u, v))=u$, which proves that $\pi_{1}$ is onto. Since $\pi_{1}$ and $f$ are continuous, what we showed is that both are homeomorphisms.

Now we can consider the product $p: U \times V \cap G^{(2)} \rightarrow U V$ and observe that for all $(g, h) \in$ $U \times V \cap G^{(2)}$, the property that $r(g h)=r(g)$ implies that

$$
\left.r\right|_{U V} \circ p=\left.r\right|_{U} \circ \pi_{1}
$$

This proves that $\left.r\right|_{U V} \circ p$ is a homeomorphism and, since $p$ is surjective, $\left.r\right|_{U V}$ is injective, therefore a bijection onto $U V$. In addition, $p$ is injective because, if $p(x)=p(y)$, by equation (2.1) $\left.r\right|_{U} \circ \pi_{1}(x)=\left.r\right|_{U} \circ \pi_{1}(y)$, hence $x=y$. Then $p$ and $\left.r\right|_{W}$ are continuous bijections such that their composition is an homeomorphism, which implies that both of them are homeomorphisms. Since $U \times V \cap G^{(2)}$ is an open set, so is $U V . r$ is injective on $U V$. The proof ends with a similar argument for $\left.s\right|_{U V}$ and we have that $U V$ is an open bisection. 


\subsection{The $*$-algebra $C_{c}(G)$}

From now we will suppose that our groupoid is LCH étale and second countable, since that is our interest in first place. The second countability is a hypothesis that [Sim] uses and so will us. On the other hand, this hypothesis is not fundamentally important for the construction that follows. Nevertheless a topological space which is LCH and secound countable is metrizable and this is a result we use plenty in Chapter 4. The metrizability comes from Urysohn's metrization theorem. The construction of the $\mathrm{C}^{*}$-algebra can be generalized even more for groupoids that are not even étale or Hausdorff. We refer to [Ren80].

Let $G$ be an étale, LCH and second countable groupoid. By the proposition $8, G_{x}$ and $G^{x}$ are discrete in $G$ for every $x \in G^{(0)}$, which makes them have a countable number of elements as the space is second countable. Let $C_{c}(G)$ denote the the space of all continuous compactly supported functions and we define a convolution operation - and a involution $*: C_{c}(G) \rightarrow C_{c}(G)$ given by

$$
\begin{aligned}
f \cdot g(\gamma) & =\sum_{\alpha \beta=\gamma} f(\alpha) g(\beta)=\sum_{\beta \in G_{s(\gamma)}} f\left(\gamma \beta^{-1}\right) g(\beta)=\sum_{\alpha \in G^{r}(\gamma)} f(\alpha) g\left(\alpha^{-1} \gamma\right) \\
f^{*}(\gamma) & =\overline{f\left(\gamma^{-1}\right)}
\end{aligned}
$$

Remark 8. We justify the second equality in (2.2), which is a change of variables. Let $\alpha \beta=\gamma$, so $s(\gamma)=s(\beta)$, which implies that $\beta \in G_{s(\gamma)}$. Since $\alpha \beta=\gamma, \alpha=\gamma \beta^{-1}$ we conclude that

$\sum_{\alpha \beta=\gamma} f(\alpha) g(\beta)=\sum_{\beta \in G_{s(\gamma)}} f\left(\gamma \beta^{-1}\right) g(\beta) \quad$ For the third equality in (2.2), the argument is analogous.

Remark 9. The sum in (2.2) is in fact finite. Denote $K=$ supp $(g)$ which is compact on $G$. We have

$$
f \cdot g(\gamma)=\sum_{\beta \in G_{s(\gamma)}} f\left(\gamma \beta^{-1}\right) g(\beta)=\sum_{\beta \in G_{s(\gamma)} \cap K} f\left(\gamma \beta^{-1}\right) g(\beta)
$$

Now, since $K$ is compact and $G_{s(\gamma)}$ is discrete, $G_{s(\gamma)} \cap K$ is finite.

We still need to show that those operations are well defined. This is the content of the next theorem. First we prove the following lemma which is really useful for calculations. We denote the functions in $C_{c}(G)$ which are supported on an open bisection by $\mathcal{C}(G)$

Lemma 3. Let $G$ be an étale groupoid which is locally compact and Hausdorff. Every element of $C_{c}(G)$ is a sum of functions in $\mathcal{C}(G)$.

Proof. Let $f$ be in $C_{c}(G)$. Using the fact that the support of $f$ is compact and that the open bisections form a neighborhood base for the topology, we may find a finite cover, $\left\{U_{i}\right\}_{i=1}^{n}$, of the support of $f$ by open bisections. Define $U_{n+1}:=G \backslash \operatorname{supp}(f)$. Since $G$ is locally compact and Hausdorff there exists a partition of unity $\left\{\alpha_{i}\right\}_{i=1}^{n+1}$ of $G$ which is subordinate to the cover $\left\{U_{i}\right\}_{i=1}^{n+1}$. Then we have

$$
f=\sum_{i=1}^{n+1} f \alpha_{i}=\sum_{i=1}^{n} f \alpha_{i}
$$

. Now just observe that $f \alpha_{i} \in \mathcal{C}(G) \quad i=1, \ldots, n$, concluding the proof.

Now we can prove that the operations of convolution and involution are well defined. This will be fundamental for the definition of the groupoid $\mathrm{C}^{*}$-algebra.

Theorem 5. Let $G$ be a $L C H$ étale groupoid that is second countable. For any $f, g \in C_{c}(G)$, the set $C_{c}(G)$ with the operations

$$
f \cdot g(\gamma)=\sum_{\alpha \beta=\gamma} f(\alpha) g(\beta)=\sum_{\beta \in G_{s(\gamma)}} f\left(\gamma \beta^{-1}\right) g(\beta)=\sum_{\alpha \in G^{r(\gamma)}} f(\alpha) g\left(\alpha^{-1} \gamma\right)
$$




$$
f^{*}(\gamma)=\overline{f\left(\gamma^{-1}\right)}
$$

is $a *$-algebra

Proof. If $f \in C_{c}(G)$ has support on a open bisection $U$, we will be using the notation that $f \in$ $\left(C_{c}(G), U\right)$. We divide the proof in the following steps

\section{- Good definition of the involution:}

Given $f \in C_{c}(G)$ we prove that $f^{*} \in C_{c}(G)$. First, suppose that $f \in\left(C_{c}(G), U\right), U$ an open bisection. That $f^{*}$ is continuous comes with ease because the complex conjugation and inversion in $G$ are both continuous. Since $f^{*}(x)=\overline{f\left(x^{-1}\right)}$, we have that

$$
\operatorname{supp}\left(f^{*}\right)=\overline{\left\{x \in G \mid f\left(x^{-1}\right) \neq 0\right\}}=: \bar{H}
$$

Given $x \in \operatorname{supp}\left(f^{*}\right)$, there is a sequence $\left(x_{i}\right)_{i}$ on $H$ such that $x_{i} \rightarrow x$ and since $x_{i} \in H$ we have $f\left(x_{i}^{-1}\right) \neq 0$. As $f\left(x_{i}^{-1}\right) \neq 0$, we have $x_{i}^{-1} \in \operatorname{supp}(f)$. By continuity $x_{i}^{-1} \rightarrow x^{-1}$, therefore $x^{-1} \in \operatorname{supp}(f) \subset U$ and we conclude that $x \in U^{-1}$. This shows that $\operatorname{supp}\left(f^{*}\right) \subset U^{-1}$.

Now we show that $\operatorname{supp}\left(f^{*}\right)$ is compact. Since $\operatorname{supp}(f)$ is compact, $\operatorname{supp}(f)^{-1}$ is compact. We have that $H \subset\left\{x^{-1} \mid f(x) \neq 0\right\} \subset \operatorname{supp}(f)^{-1}$ and so $\operatorname{supp}\left(f^{*}\right)=\bar{H}$ is a closed set inside a compact set, then $\operatorname{supp}\left(f^{*}\right)$ is compact.

We have shown that for $f \in \mathcal{C}(G), f^{*} \in \mathcal{C}(G)$. For $f \in C_{c}(G), f=\sum_{i} f_{i}$ and $f_{i} \in \mathcal{C}(G)$, which implies that $f^{*}=\sum_{i} f_{i}^{*}, f_{i}^{*} \in \mathcal{C}(G)$. We conclude that $f^{*} \in C_{c}(G)$ because $C_{c}(G)$ is a linear space.

Now we take a look at the convolution. We need that $f \cdot g$ has compact support and is continuous if both $f$ and $g$ are.

\section{- $f \cdot g$ has compact support:}

Let $\operatorname{supp}(f) \cdot \operatorname{supp}(g)$ denote the set $\{\gamma \in G: \gamma=\alpha \beta, \alpha \in \operatorname{supp}(f), \beta \in \operatorname{supp}(g)\}$. Note that this set is compact as $p(\operatorname{supp}(f) \times \operatorname{supp}(g))=\operatorname{supp}(f) \cdot \operatorname{supp}(g)$, where $p$ is the composition map on $G$. We claim that $\operatorname{supp}(f \cdot g) \subseteq \operatorname{supp}(f) \cdot \operatorname{supp}(g)$. Let $\gamma \in \operatorname{supp}(f \cdot g)$. Then,

$$
\begin{gathered}
\sum_{\alpha \beta=\gamma} f(\alpha) g(\beta) \neq 0 \Longrightarrow \exists \alpha, \beta \quad \alpha \beta=\gamma \quad \text { and } \quad f(\alpha) g(\beta) \neq 0 \\
\Longrightarrow f(\alpha) \neq 0 \text { and } g(\beta) \neq 0 \Longrightarrow \alpha \in \operatorname{supp}(f), \quad \beta \in \operatorname{supp}(g) .
\end{gathered}
$$

So $\gamma \in \operatorname{supp}(f) \cdot \operatorname{supp}(g)$. It means that $\operatorname{supp}(f \cdot g)$ is a closed subset of a compact set and, since the topology is Hausdorff, we conclude that $\operatorname{supp}(f \cdot g)$ is compact.

- Given $f \in\left(C_{c}(G), U\right)$ and $g \in\left(C_{c}(G), V\right)$, then $f \cdot g \in\left(C_{c}(G), U V\right)$ : Let $\gamma \in G$ and $f \cdot g(\gamma) \neq 0$. There are $x, y \in G$ such that $x y=\gamma$ and $f(x), g(y) \neq 0$, which by hypothesis on the support guarantees $x \in U$ and $y \in V$ and that implies $\gamma \in U V$. Since $r(\gamma)=r(x)$ and $x \in U$, an open bisection, we can write $x=\left.r\right|_{U} ^{-1}(r(\gamma))$. By the same argument we also have $y=\left.s\right|_{V} ^{-1}(s(\gamma))$.

In fact for $\gamma \in U V$ there are unique $x \in U$ and $y \in V$ such that $x y=\gamma$, which are given by the above formula. We conclude that

$$
f \cdot g(\gamma)=f\left(\left.r\right|_{U} ^{-1}(r(\gamma))\right) g\left(\left.s\right|_{V} ^{-1}(s(\gamma))\right), \quad \gamma \in U V
$$

and the expression is zero for $\gamma \notin U V$. Since the functions $f, g, r, s$ are continuous (and the inverse of $r, s$ are continuous on the appropriate domain) we observe that $\left.f \cdot g\right|_{U V}$ is continuous. Our job is done when proving continuity on all domain. On the proof that $f \cdot g$ has compact support, we have shown that $\operatorname{supp}(f \cdot g) \subset \operatorname{supp}(f) \cdot \operatorname{supp}(g) \subset U V$. From now on we define 
$A:=\operatorname{supp}(f)$ and $B:=\operatorname{supp}(g)$. Given $x \in G$ and a sequence $x_{i} \rightarrow x, x \notin U V$ implies $x \in G \backslash A B$ and the latter is an open set, meaning that $x_{i} \in G \backslash A B$ for large enough $i$. We conclude for $x \notin U V$ that $f \cdot g(x)=0=\lim _{i} f \cdot g\left(x_{i}\right)$. Now, if $x \in U V, x_{i} \in U V$ for large enough $i$ since it is open, by continuity on $U V$ we conclude $f \cdot g\left(x_{i}\right) \rightarrow f \cdot g(x)$. This is true now for every $x \in G$, so $f \cdot g$ is continuous with support on $U V$.

- $f \cdot g \in C_{c}(G)$ :

Now consider any $f, g \in C_{c}(G)=\operatorname{span\mathcal {C}}(G)$, so $f=\sum_{i} f_{i}$ and $g=\sum_{j} g_{j}, f_{i} \in\left(C_{c}(G), U_{i}\right)$ and $g_{j} \in\left(C_{c}(G), V_{j}\right)$. By linearity $f \cdot g=\sum_{i, j} f_{i} \cdot g_{j}$ and by the item we proved above this one, we have $f_{i} \cdot g_{j} \in\left(C_{c}(G), U_{i} V_{j}\right)$. As $C_{c}(G)$ is a vector space, $f \cdot g \in C_{c}(G)$.

\section{- $*$-algebra structure:}

Let $f, g, h \in C_{c}(G)$. That $\left(f^{*}\right)^{*}=f$ is pretty evident, knowing that $\left(\gamma^{-1}\right)^{-1}=\gamma$ for $\gamma \in G$.

To prove that $(f \cdot g)^{*}=g^{*} \cdot f^{*}$, let $\gamma \in G$,

$$
\begin{aligned}
(f \cdot g)^{*}(\gamma)=\overline{f \cdot g\left(\gamma^{-1}\right)}=\sum_{\alpha \beta=\gamma^{-1}} \overline{f(\alpha) g(\beta)}=\sum_{\beta^{-1} \alpha^{-1}=\gamma} g^{*}\left(\beta^{-1}\right) f^{*}\left(\alpha^{-1}\right) & =\sum_{x y=\gamma} g^{*}(x) f^{*}(y) \\
& =\left(g^{*} \cdot f^{*}\right)(\gamma) .
\end{aligned}
$$

Now let us prove the associativity of the convolution. Let $\gamma \in G$ :

$$
(f \cdot g) \cdot h(\gamma)=\sum_{a b=\gamma} f \cdot g(a) h(b)=\sum_{a b=\gamma}\left(\sum_{c d=a} f(c) g(d)\right) h(b)=\sum_{c d b=\gamma} f(c) g(d) h(b)
$$

On the other hand:

$$
f \cdot(g \cdot h)(\gamma)=\sum_{a b=\gamma} f(a)(g \cdot h)(b)=\sum_{a b=\gamma} f(a)\left(\sum_{c d=b} g(c) h(d)\right)=\sum_{a c d=\gamma} f(a) g(c) h(d)
$$

We conclude that $(f \cdot g) \cdot h=f \cdot(g \cdot h)$.

Using the ${ }^{*}$-algebra $C_{c}(G)$, we present first the Full Groupoid $\mathrm{C}^{*}$-Algebra.

\subsection{The Full Groupoid C*-algebra}

Here in this section, one of our main concerns will be studying the representations of $C_{c}(G)$ on a Hilbert space, i.e the *-homeomorphisms from $C_{c}(G)$ as a *-algebra to the $\mathrm{C}^{*}$-Algebra $\mathcal{B}(H)$, the bounded linear operators on a Hilbert space $H$.

Proposition 12. Let $G$ be a LCH étale and second countable groupoid with $G^{(0)}$ its unit space. $C_{c}\left(G^{(0)}\right)$, as a set, is a union of $C^{*}$-algebras.

Proof. Let $K$ be a compact set in $G^{(0)}$. We define

$$
C_{K}:=\{f: G \rightarrow \mathbb{C} \mid \operatorname{supp}(f) \subset K \text { and } f \text { is continuous }\} .
$$

Next, we equip this set with point-wise multiplication and the involution given by the complex conjugation. Endowing it with the natural norm $\|f\|_{\infty}=\sup _{x \in K}|f(x)|, C_{K}$ is a $\mathrm{C}^{*}$-algebra. Indeed the completeness comes from the fact that all of its elements have support on the same compact $K$. Here, we remember that the unit space $G^{(0)}$ is open and closed on $G$, in such a way that every 
function $f$ on $C_{c}\left(G^{(0)}\right)$ has a simple extension on $C_{c}(G)$, which is $f_{1}(x)=0$ for $x \in G \backslash G^{(0)}$ and $f_{1}(x)=f(x)$ for $x \in G^{(0)}$. We identify $f$ and $f_{1}$. Hence, we shall think $C_{c}\left(G^{(0)}\right)$ as a subset of $C_{c}(G)$. Now we claim that

$$
C_{c}\left(G^{(0)}\right)=\bigcup_{f \in C_{c}\left(G^{(0)}\right)} C_{\text {supp }(f)}
$$

This proves our proposition since $C_{K}$ is a $\mathrm{C}^{*}$-algebra as we said above. Let $f \in C_{c}\left(G^{(0)}\right)$, then $f \in C_{\text {supp }(f)}$ and it proves one inclusion. If $h \in \bigcup_{f \in C_{c}\left(G^{(0)}\right)} C_{\operatorname{supp}(f)}$ then $h \in C_{\text {supp }(f)}$ for a $f \in C_{c}\left(G^{(0)}\right)$, which implies that $\operatorname{supp}(h) \subset \operatorname{supp}(f)$. As the later is compact, $\operatorname{supp}(h)$ is compact. As $\operatorname{supp}(f)$ is a subset of $G^{(0)}$ we conclude so it is $\operatorname{supp}(h)$.

Proposition 12 is important since it will permit us to prove the inequality $\|\pi(f)\| \leq\|f\|_{\infty}$ for every $f \in \mathcal{C}(G)$ and $\pi$ a representation of $C_{c}(G)$. This is not trivial because $C_{c}(G)$ is not a $\mathrm{C}^{*}$-algebra. Now let $f \in \mathcal{C}(G)$ and $U$ the bisection it is supported. We have already seen that $f^{*}$ has support on $U^{-1}$, hence $f \cdot f^{*} \in C_{c}\left(U U^{-1}\right) \subset C_{c}\left(G^{(0)}\right)$. By proposition 12 , we have that $f \cdot f^{*}$ is in one $\mathrm{C}^{*}$-algebra $B$. If $\pi$ is a representation of $C_{c}(G),\left.\pi\right|_{B}: B \rightarrow \mathcal{B}(H)$ is a representation of the $B$. We have that

$$
\left\|\pi\left(f \cdot f^{*}\right)\right\| \leq\left\|f \cdot f^{*}\right\|_{\infty} \Longrightarrow\|\pi(f)\|^{2}=\left\|\pi\left(f \cdot f^{*}\right)\right\| \leq\left\|f \cdot f^{*}\right\|_{\infty} .
$$

Now we need to see what is $\left\|f \cdot f^{*}\right\|_{\infty}$ in terms of $\|f\|_{\infty}$ if $f \in \mathcal{C}(G)$. We basically need to verify what is the value of $f \cdot f^{*}(x)$ for $x \in G$. Consider $U$ to be the open bisection such that $\operatorname{supp}(f) \subset U$. By the convolution formula we have $f \cdot f^{*}(x)=\sum_{y z=x} f(y) \overline{f\left(z^{-1}\right)}$. Considering $x \in G$ such that $f \cdot f^{*}(x) \neq 0$, this sum trivialize to only one pair $(y, z)$ such that $y z=x$ and $f(y), f\left(z^{-1}\right) \neq 0$, because $U$ is an open bisection. We see that $y, z^{-1} \in U$. If there was another pair $\left(y_{1}, z_{1}\right)$ in the same conditions, clearly $r(y)=r(x)=r\left(y_{1}\right)$ and since $y, y_{1} \in U$ and $r$ is injective there, we conclude $y=y_{1}$. A similar argument can be used for $z$ and $z_{1}$.

We can see that in fact $y=\left.r\right|_{U} ^{-1}(r(x))$ and $s(y)=r(z)=s\left(z^{-1}\right)$ which implies that $y=z^{-1}$. Finally, we conclude that for every $x \in G$ such that $f \cdot f^{*}(x) \neq 0$ there exists a unique $y \in G$ such that $f \cdot f^{*}(x)=f(y) \overline{f(y)}=|f(y)|^{2}$ and so

$$
\left\|f \cdot f^{*}\right\|_{\infty}=\sup _{x \in G}\left|f \cdot f^{*}(x)\right|=\sup _{x \in U}|f(x)|^{2}=\|f\|_{\infty}^{2} .
$$

The last equality is a consequence of $\operatorname{supp}(f) \subset U$. We conclude that for $f \in \mathcal{C}(G)$ and $\pi$ a representation of $C_{c}(G)$ that $\|\pi(f)\| \leq\|f\|_{\infty}$.

Proposition 13. Let $G$ be a $L C H$ étale and second countable groupoid. For each $f \in C_{c}(G)$, there is a constant $K_{f} \geq 0$ such that $\|\pi(f)\| \leq K_{f}$ for every ${ }^{*}$-representation $\pi: C_{c}(G) \rightarrow \mathcal{B}(H)$ of $C_{c}(G)$ on a Hilbert space. If $f$ is supported on a bisection, we can take $K_{f}=\|f\|_{\infty}$.

Proof. Take $f \in C_{c}(G)$. By the lemma 3 we have $f=\sum_{i=1}^{n} f_{i}$, where $f_{i}$ is supported on a bisection. We define then $K_{f}:=\sum_{i=1}^{n}\left\|f_{i}\right\|_{\infty}$. Now take $\pi$ a ${ }^{*}$-representation of $C_{c}(G)$ and it is true that $\left\|\pi\left(f_{i}\right)\right\| \leq\left\|f_{i}\right\|_{\infty}$.

It is clear then that $\|\pi(f)\|=\left\|\sum_{i} \pi\left(f_{i}\right)\right\| \leq \sum_{i}\left\|f_{i}\right\|_{\infty}=K_{f}$, which proves the statement.

Definition 22. Let $G$ be an $L C H$ étale and second countable groupoid and $f \in C_{c}(G)$. We define

$$
\|f\|_{u}:=\sup _{\pi \text { rep. }}\|\pi(f)\|
$$

We define the full $C^{*}$-algebra of $G$, denoted by $C^{*}(G)$ by being the completion of $C_{c}(G)$ by the "norm" $\|\cdot\|_{u}$, i.e:

$$
C^{*}(G):={\overline{C_{c}(G)}}^{\|\cdot\|_{u}}
$$


Remark 10. Observe that we put in quotes the word norm on the previous definition. The reason for that is that at this point of the work, we only know that $\|\cdot\|_{u}$ is a $C^{*}$-seminorm. In the next section, we prove the existence of a representation of $C_{c}(G)$ that is faithful, therefore showing that $\|\cdot\|_{u}$ is indeed a norm.

Before we proceed to the next section, there is an important remark to be made about the definition the above $\mathrm{C}^{*}$-algebra. At first, it does not agrees with Renault's definition. This is because, in his book [Ren80], he deals with a more general framework, the non-étale case. In the non-étale case, Renault defines the full norm, not as the supremum over all *-representations of $C_{c}(G)$, but as the supremum only over *-representations of $C_{c}(G)$ that are bounded with respect to the " $I$-norm" on $C_{c}(G)$. If $G$ is étale, the $I$-norm is given by

$$
\|f\|_{I}=\sup _{x \in G^{(0)}} \max \left\{\sum_{\gamma \in G_{x}}|f(\gamma)|, \sum_{\gamma \in G^{x}}|f(\gamma)|\right\} .
$$

Renault shows that it is equivalent to say that a ${ }^{*}$-representation is bounded in the $I$-norm and that it is continuous in the inductive-limit topology on $C_{c}(G)$, i.e, the topology obtained by regarding $C_{c}(\mathcal{G})$ as the inductive limit of the subspaces $X_{K}:=\left(\{f \in C(G) \mid \operatorname{supp}(f) \subseteq K\},\|\cdot\|_{\infty}\right)$ indexed by compact subsets $K$ of $G$, we refer to [Con90] if one is not familiar with this topology and its properties. It is in Chapter IV, section 5.

So to be sure we are talking about the same $C^{*}$-algebra as Renault, we must verify that every *-representation of $C_{c}(\mathcal{G})$ is continuous in the inductive-limit topology when $G$ is étale; and for completeness we should also prove that continuity in this topology implies boundedness with respect to the $I$-norm, which shows that both definitions agree on our setting.

Lemma 4. Suppose that $G$ is $L C H$ étale groupoid. Then every ${ }^{*}$-representation $\pi$ of $C_{c}(G)$ is both continuous in the inductive-limit topology, and bounded in the I-norm.

Proof. Fix a ${ }^{*}$-representation of $C_{c}(G)$. By Proposition 5.7 on [Con90], to see that $\pi$ is continuous in the inductive-limit topology, we just have to check that $\left.\pi\right|_{X_{K}}$ is continuous for each compact $K \subseteq G$. Now, let $K \subseteq G$ be compact. Since the range and source functions are local homeomorphisms we can cover $K$ by open bisections, and then use compactness to obtain a finite subcover $K \subseteq \bigcup_{i=1}^{n} U_{i}$, each $U_{i}$ an open bisection. Fix a partition of unity $\left\{h_{i}\right\}$ for $K$ subordinate to the $U_{i}$. Then for $f \in X_{K}$, Proposition 13 gives

$$
\|\pi(f)\|=\left\|\sum_{i} \pi\left(h_{i} f\right)\right\| \leq \sum_{i=1}^{n}\left\|\pi\left(h_{i} f\right)\right\| \leq \sum_{i=1}^{n}\left\|h_{i} f\right\|_{\infty} \leq\left(\sum_{i=1}^{n}\left\|h_{i}\right\|\right)\|f\|_{\infty} \leq n\|f\|_{\infty} .
$$

So $\pi$ is Lipschitz on $X_{K}$ with Lipschitz constant at most $n$, which shows continuity on $X_{K}$ for every $K$ and by what we discussed above, $\pi$ is continuous in the inductive-limit topology.

To see that it is $I$-norm bounded, observe that if $f \in C_{c}(G)$, then for any $\gamma \in G, \gamma \in G_{s(\gamma)} \cap G^{r(\gamma)}$ and clearly,

$$
|f(\gamma)| \leq \max \left\{\sum_{\gamma \in G_{s(\gamma)}}|f(\gamma)|, \sum_{\gamma \in G^{r(\gamma)}}|f(\gamma)|\right\} .
$$

With that in mind, we have $\|f\|_{\infty} \leq\|f\|_{I}$. So the inductive-limit topology is weaker than the $I$-norm topology, and we deduce that $\pi$ is continuous for the $I$-norm. Since continuity is equivalent to boundedness for linear maps on normed spaces, we deduce that $\pi$ is $I$-norm bounded. For completeness, we now show that such bound is less or equal to 1 , i.e, that $\|\pi(f)\| \leq\|f\|_{I}$. For that, we first observe that $\|\cdot\|_{I}$ is a ${ }^{*}$-algebra norm, so $\pi$ extends to a ${ }^{*}$-homomorphism from the Banach *-algebra completion ${\overline{C_{c}(G)}}^{I}$ of $C_{c}(G)$ in the $I$-norm into $\mathcal{B}(\mathcal{H})$. Write $\rho_{A}: A \rightarrow[0, \infty)$ for the spectral-radius function on a Banach algebra $A$. Now, since $\pi$ is a ${ }^{*}$-homeomorphism, it is clear that it does not enlarge spectra of self-adjoint elements, i.e, If $y \in{\overline{C_{c}(G)}}^{I}$ is a self adjoint element, then $\operatorname{sp}(\pi(y)) \backslash\{0\} \subseteq \operatorname{sp}(y) \backslash\{0\}$. 
With that in mind, for each $f \in C_{c}(\mathcal{G})$, we have, observing that $f^{*} \cdot f$ is self adjoint,

$$
\left.\|\pi(f)\|^{2}=\left\|\pi\left(f^{*} \cdot f\right)\right\|=\rho_{\mathcal{B}(\mathcal{H})}\left(\pi\left(f^{*} \cdot f\right)\right) \leq \rho_{\overline{C_{c}(G)}} I f^{*} \cdot f\right) \leq\left\|f^{*} \cdot f\right\|_{I} \leq\|f\|_{I}^{2} .
$$

This concludes the proof.

\subsection{The Reduced Groupoid C*-algebra}

Here we proceed to show a faithful representation of $C_{c}(G)$, called the regular representation.

Eventually we are going to use the following fact.

Proposition 14. For any locally compact Hausdorff space X, let

$$
C_{0}(X):=\{f \in C(X): \forall \epsilon>0 \text { the set }\{x:|f(x)| \geq \epsilon\} \quad \text { is compact }\}
$$

With pointwise addition, multiplication, complex conjugation and supremum norm $C_{0}(X)$ is a $C^{*}$ algebra.

We start by taking $u \in G^{(0)}$. With that consider the Hilbert space $\ell^{2}\left(s^{-1}(u)\right)$ of functions from $s^{-1}(u)$ to $\mathbb{C}$ that are square summable. As a reminder we write its definition, namely

$$
\ell^{2}\left(s^{-1}(u)\right)=\left\{\zeta: s^{-1}(u) \rightarrow \mathbb{C}: \sum_{x \in s^{-1}(u)}|\zeta(x)|^{2}<\infty\right\}
$$

The inner product is clearly given by $\langle\zeta, \eta\rangle=\sum_{x \in s^{-1}(u)} \zeta(x) \overline{\eta(x)}$. We remember as well the notation $s^{-1}(u)=G_{u}$ and note that, since $G$ is second countable, $G_{u}$ is countable by proposition 8. We now define $\pi_{\lambda}^{u}: C_{c}(G) \rightarrow \mathcal{B}\left(\ell^{2}\left(G_{u}\right)\right)$ by

$$
\pi_{\lambda}^{u}(f) \zeta(x):=\sum_{y: r(y)=r(x)} f(y) \zeta\left(y^{-1} x\right), \quad f \in C_{c}(G) \quad \zeta \in \mathcal{B}\left(\ell^{2}\left(G_{u}\right)\right) \quad x \in \ell^{2}\left(G_{u}\right)
$$

There is some work to do here. First we need to prove that this function is well defined and is a representation of $C_{c}(G)$ to $\mathcal{B}\left(\ell^{2}\left(G_{u}\right)\right)$. In order to prove these claims, define for each $\gamma \in G^{(0)}$ the element

$$
\delta_{\gamma}(\eta)=\left\{\begin{array}{l}
1, \gamma=\eta \\
0, \gamma \neq \eta
\end{array}\right.
$$

which belongs to $\ell^{2}\left(G_{u}\right)$. Moreover, the set $\left\{\delta_{\gamma}: \gamma \in G^{(0)}\right\}$ is an orthonormal basis for $\ell^{2}\left(G_{u}\right)$, therefore the function $\pi_{\lambda}^{u}$ could have been defined only on those elements.

Proposition 15. $\pi_{\lambda}^{u}$ is a representation of $C_{c}(G)$.

\section{Proof. • Good Definition of $\pi_{\lambda}^{u}$}

First, the sum $\sum_{y: r(y)=r(x)} f(y) \zeta\left(y^{-1} x\right)$ is finite on a similar argument we made for the definition of the convolution product on $C_{c}(G)$. Let $f \in \mathcal{C}(G)$. It is not difficult to verify that $\pi_{\lambda}^{u}(f)$ is a linear operator. We prove that $\left\|\pi_{\lambda}^{u}(f) \zeta\right\| \leq\|f\|_{\infty}\|\zeta\|$. For that, consider $U$ to be the open bisection which $f$ is supported. We shall see how the formula for $\pi_{\lambda}^{u}$ reduces for such functions,

$$
\pi_{\lambda}^{u}(f) \zeta(x)=\sum_{y: r(y)=r(x)} f(y) \zeta\left(y^{-1} x\right)
$$

The sum is on $y \in r^{-1}(r(x)) \cap \operatorname{supp}(f), x \in G_{u}$, since the points where $f$ is zero does not concern us. This set, in fact, contains at most one element. Let $y_{1}, y_{2} \in r^{-1}(r(x)) \cap \operatorname{supp}(f)$. Then $r\left(y_{1}\right)=r\left(y_{2}\right)=r(x)$ and both are in $U$, since $r$ is a homeomorphism in such set $y_{1}=y_{2}$. We write $y=y_{x}$ for the unique element in $r^{-1}(r(x)) \cap \operatorname{supp}(f)$ with $x \in G_{u}$. We have 


$$
\begin{gathered}
\pi_{\lambda}^{u}(f) \zeta(x)=\left\{\begin{array}{l}
f\left(y_{x}\right) \zeta\left(y_{x}^{-1} x\right), \text { If } r^{-1}(r(x)) \cap \operatorname{supp}(f) \neq \emptyset \\
0, \text { otherwise }
\end{array} .\right. \\
\left\|\pi_{\lambda}^{u}(f) \zeta\right\|^{2}=\sum_{x \in G_{u}}\left|f\left(y_{x}\right) \zeta\left(y_{x}^{-1} x\right)\right|^{2} \leq\|f\|_{\infty}^{2} \sum_{x \in G_{u}}\left|\zeta\left(y_{x}^{-1} x\right)\right|^{2} \leq\|f\|_{\infty}^{2}\|\zeta\|^{2}
\end{gathered}
$$

This proves that $\left\|\pi_{\lambda}^{u}(f)\right\| \leq\|f\|_{\infty}<\infty$ for $f \in \mathcal{C}(G)$, but by linearity on $f$ (which is easy to verify) we have as well $\left\|\pi_{\lambda}^{u}(f)\right\|<\infty$ for all $f \in C_{c}(G)$, proving that $\pi_{\lambda}^{u}(f) \in \mathcal{B}\left(\ell^{2}\left(G_{u}\right)\right)$ which proves that $\pi_{\lambda}^{u}$ is well defined.

- Compatibility with involution, i.e $\pi_{\lambda}^{u}\left(f^{*}\right)=\pi_{\lambda}^{u}(f)^{*}$

For this calculation and the next item we shall be using the basis of $\ell^{2}\left(G_{u}\right)$ given by $\delta_{x}$. Let us calculate $\pi_{\lambda}^{u}$ in this basis.

$$
\pi_{\lambda}^{u}(f) \delta_{x}(h)=\sum_{y: r(y)=r(x)} f(y) \delta_{x}\left(y^{-1} h\right)=f\left(h x^{-1}\right)
$$

And that implies,

$$
\pi_{\lambda}^{u}(f) \delta_{x}=\sum_{h: s(h)=u} f\left(h x^{-1}\right) \delta_{h}
$$

We, then, can calculate

$$
\begin{aligned}
\left\langle\pi_{\lambda}^{u}\left(f^{*}\right) \delta_{x_{1}}, \delta_{x_{2}}\right\rangle=\left\langle\sum_{h: s(h)=u} f^{*}\left(h x_{1}^{-1}\right) \delta_{h}, \delta_{x_{2}}\right\rangle & =\sum_{h: s(h)=u} \overline{f\left(x_{1} h^{-1}\right)}\left\langle\delta_{h}, \delta_{x_{2}}\right\rangle \\
& =\overline{f\left(x_{1} x_{2}^{-1}\right)}=\sum_{h: s(h)=u} \overline{f\left(h x_{2}^{-1}\right)}\left\langle\delta_{x_{1}}, \delta_{h}\right\rangle \\
& =\left\langle\delta_{x_{1}}, \sum_{h: s(h)=u} f\left(h x_{2}^{-1}\right) \delta_{h}\right\rangle=\left\langle\delta_{x_{1}}, \pi_{\lambda}^{u}(f) \delta_{x_{2}}\right\rangle .
\end{aligned}
$$

As $x_{1}$ and $x_{2}$ are arbitrary elements of $G_{u}$, we conclude that $\pi_{\lambda}^{u}\left(f^{*}\right)=\pi_{\lambda}^{u}(f)^{*}$.

- Multiplicative property, i.e $\pi_{\lambda}^{u}(f \cdot g)=\pi_{\lambda}^{u}(f) \pi_{\lambda}^{u}(g)$

Consider again $\delta_{x}$. We already proved that

$$
\pi_{\lambda}^{u}(f) \delta_{x}=\sum_{y: s(y)=u} f\left(y x^{-1}\right) \delta_{y}
$$

Now, using the above equality, changing $f \rightarrow f \cdot g$ and using the convolution product third equality in (2.2), we have the expression

$$
\pi_{\lambda}^{u}(f \cdot g) \delta_{x}=\sum_{y: s(y)=u}\left(\sum_{k: r(k)=r\left(y x^{-1}\right)=r(y)} f(k) g\left(k^{-1} y x^{-1}\right)\right) \delta_{y}
$$

Using (2.4) and linearity of $\pi_{\lambda}^{u}(f)$ we have

$$
\pi_{\lambda}^{u}(f) \pi_{\lambda}^{u}(g) \delta_{x}=\sum_{y: s(y)=u}\left(w: \sum_{s(w)=u} f\left(y w^{-1}\right) g\left(w x^{-1}\right)\right) \delta_{y}
$$


What it is needed to do here is to show that in fact $\pi_{\lambda}^{u}(f) \pi_{\lambda}^{u}(g) \delta_{x}=\pi_{\lambda}^{u}(f \cdot g) \delta_{x}$ with those two expressions at hand.

Let $y \in G$ such that $s(y)=u$. We define the function $U_{y}: s^{-1}(u) \rightarrow r^{-1}(r(y))$ by $x \mapsto y x^{-1}$. One can verify that it is a bijection and the inverse $U_{y}^{-1}: r^{-1}(r(y)) \rightarrow s^{-1}(u)$ is given by the map $k \mapsto k^{-1} y$.

With all of this in hand, we have:

$$
\begin{aligned}
\sum_{w: s(w)=u} f\left(y w^{-1}\right) g\left(w x^{-1}\right)=\sum_{w: s(w)=u} f\left(U_{y}(w)\right) g\left(U_{y}^{-1} U_{y}(w) x^{-1}\right) & =\sum_{k: r(k)=r(y)} f(k) g\left(U_{y}^{-1}(k) x^{-1}\right) \\
& =\sum_{k: r(k)=r(y)} f(k) g\left(k^{-1} y x^{-1}\right)
\end{aligned}
$$

And this finally proves that $\pi_{\lambda}^{u}(f) \pi_{\lambda}^{u}(g) \delta_{x}=\pi_{\lambda}^{u}(f \cdot g) \delta_{x}$, since the two expressions coincide. We conclude then that $\pi_{\lambda}^{u}$ is a ${ }^{*}$-homomorphism.

For $f \in C_{c}(G)$, we showed in the prove above that, for $x \in G_{u}$,

$$
\pi_{\lambda}^{u}(f) \delta_{x}=\sum_{y: s(y)=u} f\left(y x^{-1}\right) \delta_{y}
$$

We, in fact, can write it in another form. Consider the functions

$$
\begin{gathered}
\phi: s^{-1}(u) \rightarrow s^{-1}(r(x)), y \mapsto y x^{-1} \\
\psi: s^{-1}(r(x)) \mapsto s^{-1}(u), h \mapsto h x
\end{gathered}
$$

They are bijections and inverse one of the other. Indeed, if $\phi\left(y_{1}\right)=\phi\left(y_{2}\right)$ we have $y x^{-1}=y_{2} x^{-1}$ which implies $y_{1}=y_{2}$. Similarly for $\psi$. They are inverses, since $\phi(\psi(z))=\phi(h x)=h x x^{-1}=z$. The other relation is analogous. We conclude that

$$
\begin{aligned}
\sum_{y: s(y)=u} f\left(y x^{-1}\right) \delta_{y} & =\sum_{y: s(y)=u} f(\phi(y)) \delta_{y} \\
& =\sum_{h: s(z)=r(x)} f(\phi(\psi(h))) \delta_{\psi(h)} \\
& =\sum_{h: s(z)=r(x)} f(h) \delta_{h x}
\end{aligned}
$$

Then, for $f \in C_{c}(G)$ and $x \in G$ such that $x \in G_{u}$ we can derive the identity

$$
\pi_{\lambda}^{u}(f) \delta_{x}=\sum_{h: s(h)=r(x)} f(h) \delta_{h x}
$$

This is going to be useful for the next proposition.

Proposition 16. Let $f \in C_{c}(G)$. Then it is true:

$$
f(y)=\left\langle\pi_{\lambda}^{s(y)}(f) \delta_{s(y)}, \delta_{y}\right\rangle
$$

for all $y \in G$.

Proof. Consider an element $y \in G$, by the considerations we've done: 


$$
\pi_{\lambda}^{u}(f) \delta_{x}=\sum_{h: s(h)=r(x)} f(h) \delta_{h x} \Longrightarrow \pi_{\lambda}^{s(y)}(f) \delta_{s(y)}=\sum_{h: s(h)=r(s(y))=s(y)} f(h) \delta_{h s(y)}=\sum_{h: s(h)=s(y)} f(h) \delta_{h}
$$

Then:

$$
\left\langle\pi_{\lambda}^{s(y)}(f) \delta_{s(y)}, \delta_{y}\right\rangle=\sum_{h: s(h)=s(y)} f(h)\left\langle\delta_{h}, \delta_{y}\right\rangle=f(y)
$$

We now have the resources to show the regular representation of $C_{c}(G)$. Each $\pi_{\lambda}^{u}$, for $u \in G^{(0)}$ is a representation of $C_{c}(G)$ on the Hilbert space $\ell^{2}\left(G_{u}\right)$. Now consider the direct sum

$$
\mathcal{S}:=\bigoplus_{u \in G^{(0)}} \ell^{2}\left(G_{u}\right)
$$

Which are the vectors $\left(x_{u}\right)$ indexed on $G^{(0)}$ such that $x_{u} \in \ell^{2}\left(G_{u}\right)$ and the $\operatorname{sum} \sum_{u \in G^{(0)}}\left\langle x_{u}, x_{u}\right\rangle_{\ell^{2}\left(G_{u}\right)}$ is finite. The inner product on $\mathcal{S}$ is

$$
\left\langle\left(x_{u}\right),\left(y_{u}\right)\right\rangle=\sum_{u \in G^{(0)}}\left\langle x_{u}, y_{u}\right\rangle_{\ell^{2}\left(G_{u}\right)}
$$

Naturally such inner product induces a norm on $\mathcal{S}$ and we can consider the completion of such space, a Hilbert space denoted by $\ell^{2}(G):=\overline{\mathcal{S}}$.

We can consider in $\mathcal{S}$, for $f \in C_{c}(G)$, the function $\pi_{\lambda}(f): \mathcal{S} \rightarrow \mathcal{S}$ which is defined as

$$
\left(x_{u}\right) \mapsto\left(\pi_{\lambda}^{u}(f)\left(x_{u}\right)\right)
$$

It is routine to verify that it is a linear operator and that it is continuous. Since $\mathcal{S}$ is dense in $\ell^{2}(G), \pi_{\lambda}(f)$ has a unique continuous extension and to not use too much notation, such extension will still be denoted by $\pi_{\lambda}(f)$.

We can finally define the regular representation of $C_{c}(G)$ by being the ${ }^{*}$-homomorphism

$$
\begin{gathered}
\pi_{\lambda}: C_{c}(G) \rightarrow \mathcal{B}\left(\ell^{2}(G)\right) \\
f \mapsto \pi_{\lambda}(f)
\end{gathered}
$$

By construction, we observe that is satisfies $\pi_{\lambda}(f)(x)=\left(\pi_{\lambda}^{u}(f)\left(x_{u}\right)\right)$ for $x \in \mathcal{S}$. Now we wish to show that such a representation is faithful.

Theorem 6. The regular representation $\pi_{\lambda}$ is faithful

Proof. Here where comes the importance of the formula on Proposition 16. Let $f \in C_{c}(G)$ such that $\pi_{\lambda}(f)=0$. Then $\pi_{\lambda}(f) x=\left(\pi_{\lambda}^{u}(f)\left(x_{u}\right)\right)=0$ for all $x \in \mathcal{S}$. Let $y \in G$ and consider the vector $x \in \mathcal{S}$ such that $x_{s(y)}=\delta_{s(y)}$ and zero otherwise. This makes sense because $\delta_{s(y)} \in \ell^{2}\left(G_{s(y)}\right)$. By the above $\pi_{\lambda}^{s(y)}(f) \delta_{s(y)}=0$ and by the formula on proposition 16 we have that $f(y)=0$. Since $y \in G$ was arbitrary, we conclude that $f=0$.

We can now define the reduced $\mathrm{C}^{*}$-algebra:

Definition 23. The reduced $C^{*}$-algebra of an $L C H$ étale second countable groupoid $G$, denoted by $C_{r}^{*}(G)$, is the completion of $C_{c}(G)$ by the norm

$$
\|f\|_{r}:=\left\|\pi_{\lambda}(f)\right\| .
$$

We are now interested in showing that the above norm can be rewritten as the supremum over the representations we used to define $\pi_{\lambda}$. 
Proposition 17. For $f \in C_{c}(G)$ :

$$
\|f\|_{r}=\sup _{u \in G^{(0)}}\left\|\pi_{\lambda}^{u}(f)\right\|
$$

Proof. We prove the two inequalities, namely $\sup _{u \in G^{(0)}}\left\|\pi_{\lambda}^{u}(f)\right\| \leq\|f\|_{r}$ and $\|f\|_{r} \leq \sup _{u \in G^{(0)}}\left\|\pi_{\lambda}^{u}(f)\right\|$.

For the first, let $x_{u} \in \ell^{2}\left(G_{u}\right)$ and define $x=\left(0, \ldots, x_{u}, \ldots\right)$, i.e the element of $\ell^{2}\left(G_{u}\right)$ such that in the $u$ component is equal to $x_{u}$. Clearly $\left\|x_{u}\right\|=\|x\|$ and by the definition of $\pi_{\lambda}$, we have $\pi_{\lambda}(f)(x)=\pi_{\lambda}^{u}(f)\left(x_{u}\right)$. With that

$$
\left\|\pi_{\lambda}^{u}(f)\left(x_{u}\right)\right\|=\left\|\pi_{\lambda}(f)(x)\right\| \leq\left\|\pi _ { \lambda } ( f ) \left|\||| x\|=\left\|\pi _ { \lambda } ( f ) \left|\left\||| x_{u}\right\|\right.\right.\right.\right.
$$

Since $\left\|\pi_{\lambda}^{u}(f)\right\|=\sup _{\left\|x_{u}\right\| \leq 1}\left\|\pi_{\lambda}^{u}(f)\left(x_{u}\right)\right\|$ we have that $\sup _{u \in G^{(0)}}\left\|\pi_{\lambda}^{u}(f)\right\| \leq\|f\|_{r}$.

Now, for the other side, consider

$$
\|f\|_{r}=\sup _{x \in \ell^{2}(G),\|x\| \leq 1}\left\|\pi_{\lambda}(f) x\right\|
$$

The supremum on the right hand side could have been done on the elements $x \in \mathcal{S}$ because $\mathcal{S}$ is dense in $\ell^{2}(G)$. Then

$$
\left\|\pi_{\lambda}(f) x\right\|^{2}=\left\|\left(\pi_{\lambda}^{u}(f)\left(x_{u}\right)\right)\right\|^{2}=\sum_{u \in G^{(0)}}\left\|\pi_{\lambda}^{u}(f) x_{u}\right\|^{2} \leq \sup _{u \in G^{(0)}}\left\|\pi_{\lambda}^{u}(f)\right\|^{2}\|x\|^{2}
$$

The above proves that $\|f\|_{r} \leq \sup _{u \in G^{(0)}}\left\|\pi_{\lambda}^{u}(f)\right\|$ and we conclude that they must be equal.

Now, since we have finally defined both $\mathrm{C}^{*}$-Algebras, we should see how they relate with each other; that is the content of the next proposition.

Proposition 18. Let $G$ be a $L C H$ étale and second countable groupoid. There exists an closed ideal I of $C^{*}(G)$ such that

$$
C^{*}(G) / I \simeq C_{r}^{*}(G)
$$

Proof. First, consider the identity function $i:\left(C_{c}(G),\|\cdot\|_{u}\right) \rightarrow C_{r}^{*}(G)$, which means that $i(f)=f$ for $f \in C_{c}(G)$. Clearly, it is a *-homomorphism and

$$
\|i(f)\|_{r}=\|f\|_{r} \leq\|f\|_{u},
$$

i.e, it is bounded. Since $\left(C_{c}(G),\|\cdot\|_{u}\right)$ is dense in $C^{*}(G)$, there is a extension $q$ of $i$ to all $C^{*}(G)$ and this extension is a ${ }^{*}$-homomorphism. We have that $C_{c}(G) \subset \operatorname{Im} q$ and, because $\operatorname{Im} q$ is an $\mathrm{C}^{*}$-algebra, we conclude that

$$
C_{r}^{*}(G)=\overline{C_{c}(G)} \|^{\|\cdot\|_{r}} \subset \operatorname{Imq} .
$$

By the above $q$ is surjective. Define $I:=\operatorname{Ker} q$, which is an closed ideal of $C^{*}(G)$ and consider the quotient $C^{*}(G) / I$. Now, $q$ induces an injective *-homomorphism of $C^{*}(G) / I$ onto $C_{r}^{*}(G)$. Indeed, for $h+I \in C^{*}(G) / I$ define

$$
q^{\prime}: C^{*}(G) / I \rightarrow C_{r}^{*}(G), \quad h+I \mapsto q(h) .
$$

It is well defined, since given $h+I \in C^{*}(G) / I$ and $g \in h+I$, i.e $g=h+f$ for $f \in I$ we have, since $f \in \operatorname{Ker} q$,

$$
q^{\prime}(h+I)=q(h)=q(h+f)=q(g)=q^{\prime}(g+I) .
$$

It is routine to verify the axioms for *-homomorphism, we prove that it is injective. Indeed, let $q^{\prime}(h+I)=q^{\prime}(g+I)$, which implies $q(h)=q(g)$ and $q(h-g)=0$, which means that $h-g \in I$. 
This proves that $h \in g+I$ and $h+I=g+I$, concluding that $q^{\prime}$ is an injective *-homomorphism beetween the two $\mathrm{C}^{*}$-Algebras $C^{*}(G) / I$ and $C_{r}^{*}(G)$, i.e, $C^{*}(G) / I \simeq C_{r}^{*}(G)$.

This is, in particular, a relevant proposition, because if we know that $C^{*}(G)$ is simple, i.e, there are no non-trivial closed ideals, then by proposition 18 we have $C^{*}(G) \simeq C_{r}^{*}(G)$. This will be the case for the groupoid we study on the next chapter.

Now we wish to see hoe the $\mathrm{C}^{*}$-Algebra $C_{0}\left(G^{(0)}\right)$ is related with those. In fact, it is a sub$\mathrm{C}^{*}$-algebra of $C_{r}^{*}(G)$ and $C^{*}(G)$. Next we introduce a conditional expectation of $C_{r}^{*}(G)\left(C^{*}(G)\right)$ onto $C_{0}\left(G^{(0)}\right)$. By Conditional expectation of a $\mathrm{C}^{*}$-Algebra $\mathfrak{A}$ onto a sub-C*-algebra $\mathfrak{B}$, we mean a positive and continuous linear map $P$, such that its restriction to $\mathfrak{B}$ is the identity in $\mathfrak{B}$ and for $a \in \mathfrak{A}, b_{1}, b_{2} \in \mathfrak{B}$, we have $P\left(b_{1} a b_{2}\right)=b_{1} P(a) b_{2}$.

Proposition 19. Let $G$ be a $L C H$, étale and second countable groupoid. For any $B \subset G$, an open bisection, there exists $j: C_{0}(B) \rightarrow C_{r}^{*}(G)$ and $h: C_{0}(B) \rightarrow C^{*}(G)$, both linear and isometric maps.

Proof. We already have, for $f \in \mathcal{C}(G)$, that $\|\pi(f)\| \leq\|f\|_{\infty}$, where $\pi$ is a representation of $C_{c}(G)$. Clearly, taking the supreme over all representations or only the regular representations we have that $\|f\|_{u} \leq\|f\|_{\infty}$ and $\|f\|_{r} \leq\|f\|_{\infty}$. First, we prove the proposition for $C_{r}^{*}(G)$. Fix $B$ an open bisection and consider the inclusion map $i: C_{c}(B) \rightarrow C_{r}^{*}(G), f \mapsto f$. It is linear and continuous, indeed the continuity comes from the fact that $i$ is bounded,

$$
\|i(f)\|_{r}=\|f\|_{r} \leq\|f\|_{\infty} .
$$

By proposition 16, for any $f \in C_{c}(G)$ and $x \in G$,

$$
|f(x)|=\left|\left\langle\pi_{\lambda}^{s(x)}(f) \delta_{s(x)}, \delta_{x}\right\rangle\right| \leq\left\|\pi_{\lambda}^{s(x)}(f)\right\| .
$$

Where we used the Cauchy-Schwartz inequality. Taking the supreme over all $x \in G$,

$$
\|f\|_{\infty} \leq \sup _{x \in G}\left\|\pi_{\lambda}^{s(x)}(f)\right\| \leq\|f\|_{r}
$$

This shows that for $f \in \mathcal{C}(G)$, the reduced norm and the uniform norm coincides. Since $\|f\|_{r} \leq$ $\|f\|_{u}$, we see that all those norms coincide when $f \in \mathcal{C}(G)$. In particular, this shows that the inclusion map $i$ is an isometry and, since $\left(C_{c}(B),\|\cdot\|_{\infty}\right)$ is dense in $C_{0}(B), i$ extends to a linear isometry from $C_{0}(B)$ to $C_{r}^{*}(G)$.

Now, for the full $C^{*}$-Algebra is similar, just consider $h: C_{c}(B) \rightarrow C^{*}(G)$ to be the inclusion map. The above considerations show that $h$ is a linear isometry and it extends to a linear isometry from $C_{0}(B)$ to $C^{*}(G)$.

Proposition 20. $C_{0}\left(G^{(0)}\right)$ with pointwise product, the usual vector space structure, with the uniform norm $\|\cdot\|_{\infty}$ and the involution $f^{*}(x)=\overline{f(x)}$ for $f \in C_{0}\left(G^{(0)}\right)$ and $x \in G^{(0)}$ is a sub-C*algebra of $C_{r}^{*}(G)$ and $C^{*}(G)$.

Proof. Clearly $G^{(0)}$ is an open bisection, since the maps r,s are the identity(therefore injective) and $G^{(0)}$ is open in $G$. By proposition 19 with $B=G^{(0)}, C_{0}\left(G^{(0)}\right)$ is a closed subspace of $C^{*}(G)$ or $C_{r}^{*}(G)$. It is left to prove that $C_{0}\left(G^{(0)}\right)$ is a sub-algebra, i.e for any $h, u \in C_{0}\left(G^{(0)}\right), h u=h \cdot u$. In other words, the pointwise product coincides with the convolution product that we defined for $C_{c}(G)$. With that in mind, let $f_{1}, f_{2} \in C_{c}\left(G^{(0)}\right)$, which we can identify with elements of $C_{c}(G)$ with support on $G^{(0)}$. For $x \in G$,

$$
f_{1} \cdot f_{2}(x)=\sum_{a b=x} f_{1}(a) f_{2}(b)
$$

Since both functions are supported on $G^{(0)}$, the above $a, b$ can be taken as elements of $G^{(0)}$. In this case the equation $x=a b$ has a unique solution, i.e, $x=a=b$. Indeed, $a=b$ because otherwise we would have $r(b)=b \neq a=s(a)$, and so $x=a=b$. With this $f_{1} \cdot f_{2}(x)=f_{1}(x) f_{2}(x)$ for $x \in G^{(0)}$, zero otherwise. We should show, as well, that the involutions are the same, indeed, this is a simple 
conclusion of the fact that for $x \in G^{(0)}, x=x^{-1}$. Proved for the dense set $C_{c}\left(G^{(0)}\right)$ of $C_{0}\left(G^{(0)}\right)$, we have finally that $C_{0}\left(G^{(0)}\right)$ is a sub-C*-algebra of $C^{*}(G)$ and $C_{r}^{*}(G)$.

Now, we talk about the conditional expectation mentioned previously. Consider for $f \in C_{c}(G)$ the restriction map $\left.f \mapsto f\right|_{G^{(0)}}$ of $C_{c}(G)$ to $C_{c}\left(G^{(0)}\right)$ which is continuous and linear. It then extends by continuity to a bounded linear map $P: C_{r}^{*}(G) \rightarrow C_{0}\left(G^{(0)}\right)$ (it could've been $C^{*}(G)$ in the place of $C_{r}^{*}(G)$ as well). First we need to prove that it is a positive map. Indeed take a positive element of $C_{c}(G)$,i.e, take $h=g^{*} \cdot g$, for $g \in C_{c}(G)$; since a positive element of $C_{0}\left(G^{(0)}\right)$ with the usual $\mathrm{C}^{*}$-algebra structure is just the positive functions, we just need to show that $\left.h\right|_{G^{(0)}}$ is a positive function. We compute, for $x \in G^{(0)}$

$$
h(x)=g^{*} \cdot g(x)=\sum_{\beta \in G_{x}} g^{*}\left(x \beta^{-1}\right) g(\beta)=\sum_{\beta \in G_{x}} g^{*}\left(\beta^{-1}\right) g(\beta)=\sum_{\beta \in G_{x}} \overline{g(\beta)} g(\beta)=\sum_{\beta \in G_{x}}|g(\beta)|^{2} \geq 0 .
$$

We have, then, proved that for $h \in C_{c}(G)$, positive in $C_{r}^{*}(G)$ (or $C^{*}(G)$ ), $P(h)=\left.h\right|_{G^{(0)}}$ is a positive element of $C_{0}\left(G^{(0)}\right)$. Since $C_{c}(G)$ is dense in $C_{r}^{*}(G)$ (or $C^{*}(G)$ ), for a positive element $p=w^{*} \cdot w \in C_{r}^{*}(G)$ there exist a sequence $\left(w_{n}\right)_{n}$ in $C_{c}(G)$ such that $w_{n} \rightarrow w$, so $p=\lim _{n} w_{n}^{*} \cdot w_{n}$ and by continuity of $P$ we have $P(p)=\lim _{n} P\left(w_{n}^{*} \cdot w_{n}\right)$. As we've done before $P\left(w_{n}^{*} \cdot w_{n}\right)$ is a positive element and since the positive elements form a closed set we finally conclude that $P(p)$ is positive for every positive element of $C_{r}^{*}(G)$, i.e, $P$ is a positive map.

Observe that $P$ restricted to $C_{0}\left(G^{0}\right)$ is the identity map, since for $f \in C_{0}\left(G^{0}\right)$ take $f_{n} \rightarrow f$, $f_{n} \in C_{c}\left(G^{(0)}\right)$, then

$$
P(f)=\lim _{n} P\left(f_{n}\right)=\left.\lim _{n} f_{n}\right|_{G^{(0)}}=\lim _{n} f_{n}=f .
$$

Now, let $f \in C_{c}(G)$ and $g_{1}, g_{2} \in C_{c}\left(G^{(0)}\right) . P\left(g_{1} \cdot f \cdot g_{2}\right)=\left.\left(g_{1} \cdot f \cdot g_{2}\right)\right|_{G^{(0)}}$.

We observe that, for $x \in G$

$$
\left(g_{1} \cdot f \cdot g_{2}\right)(x)=\left(g_{1} \cdot\left(f \cdot g_{2}\right)\right)(x)=g_{1}(r(x))\left(f \cdot g_{2}\right)(x)=g_{1}(r(x)) f(x) g_{2}(s(x)) .
$$

If $x \in G^{(0)}$ then, $\left(g_{1} \cdot f \cdot g_{2}\right)(x)=g_{1}(x) f(x) g_{2}(x)=\left(g_{1} P(f) g_{2}\right)(x)$. This proves that $P\left(g_{1} \cdot f \cdot g_{2}\right)=$ $g_{1} \cdot P(f) \cdot g_{2}$ for $f \in C_{c}(G)$ and $g_{i} \in C_{c}\left(G^{(0)}\right)$. The conclusion for $a \in C_{r}^{*}(G)$ and $b_{i} \in C_{0}\left(G^{(0)}\right)$ $i=1,2$ comes from density and continuity of $P$. All this shows that $P: C_{r}^{*}(G) \rightarrow C_{0}\left(G^{(0)}\right)$ is a conditional expectation. 


\section{Chapter 3}

\section{Conformal Measures}

\subsection{Conformal Measures}

Here we introduce the notion of conformal measure as in [DU91]. We will be showing, in a certain context, how these measures relate to $\mathrm{C}^{*}$-algebras and KMS states.

Consider a measurable endomorphism $T: X \rightarrow X$ on a measurable space $(X, \mathcal{F})$ and a measurable nonnegative function $f$ on $X$.

Definition 24. A measure $m$ on $(X, \mathcal{F})$ is called $f$-conformal, if

$$
m(T(A))=\int_{A} f(z) m(d z)
$$

whenever $A \in \mathcal{F}$ is a measurable set, for which $T(A)$ is measurable and $T: A \rightarrow T(A)$ is invertible. Such kinds of set are called special sets.

First we present results that relates these kind of measures with the Perron Frobenius operator, that we introduced in a particular seeting in Chapter 1.

Proposition 21. Let $m$ be a $f$-conformal measure, then $m \circ T$ is absolutely continuous with respect to $m$ on the $\sigma$-algebra $\mathcal{F} \cap A$ for every special set $A$ such that $T: A \rightarrow T(A)$ is a measurable isomorphism.

Proof. We first prove that $m \circ T$ is indeed a measure. $f$ is nonnegative, so $m \circ T(C) \geq 0$. Let $\left\{B_{n}\right\}_{n \in \mathbb{N}}$ a sequence of sets of $\mathcal{F} \cap A$, that are disjoint, then, obviously $m \circ T(\emptyset)=0$ and

$$
m \circ T\left(\cup B_{n}\right)=m\left(T\left(\cup B_{n}\right)\right)=m\left(\cup T\left(B_{n}\right)\right)=\sum m \circ T\left(B_{n}\right)
$$

Where in the equality it was used that $T$ is injective. Let $B \in \mathcal{F} \cap A$ so that $m(B)=0$. To prove that $m \circ T \ll m$ let $f=\sum_{j=1}^{n} a_{j} \chi_{E_{j}}$ a simple function, then

$$
m \circ T(B) \stackrel{3.1}{=} \sum a_{j} m\left(E_{j} \cap B\right)=0
$$

For $f$ measurable non negative just use the monotone convergence theorem.

The last proposition show us that (3.1) is equivalent to the fact that the Radon-Nikodym derivative $\frac{d m \circ T}{d m}=f_{\mid A}$. Let $m$ be an $f$-conformal measure and define $f^{(1)}=f$ and $f^{(n)}=\left(f^{(n-1)} \circ\right.$ $T) f$. From (3.1), $m$ is also $f^{(n)}$-conformal with respect to the transformation $T^{n}$ and

$$
\int_{T A} g d m=\int_{A}(g \circ T) f d m
$$

for any measurable function $g$, which is nonnegative and integrable on $T(A)$. To prove it just prove it for $g$ a characteristic function and the rest goes by usual means(linearity and monotone convergence theorem). 
Let $T$ be a measurable endomorphism $(X, \mathcal{F})$, which is finite-to-one, i.e $\exists n \in \mathbb{N}$ such that $\forall x \in X$ $n \geq\left|T^{-1}(x)\right|$. For a measurable function $f: X \rightarrow R_{+}$the Perron-Frobenius operator:

$$
L_{f} \varphi(x)=L \varphi(x)=\sum_{T(y)=x} \frac{\varphi(y)}{f(y)}
$$

is well defined for measurable functions $\varphi$ and all $x \in X$ such that $f(y)>0$ for all preimages $y$ of $x$.

Theorem 7. Assume that there exists a finite partition of $X$ into special sets $X_{i}(1 \leq i \leq s)$, such that $T: X_{i} \rightarrow T\left(X_{i}\right)$ is a measurable isomorphism. Let $f: X \rightarrow R_{+}$be a measurable function and $m$ be a probability measure on $(X, \mathcal{F})$. Then $m$ is $f$-conformal if and only if $L_{f}$ acts on $L^{1}(m)$ and $L^{*} m=m$.

Proof. Suppose $m$ is $f$-conformal. If $\varphi$ is an integrable function, (3.2) implies that:

$$
\int_{T\left(X_{i}\right)} \frac{\varphi}{f} \circ\left(T_{\mid X_{i}}\right)^{-1} d m=\int_{X_{i}} \varphi d m
$$

summing over $i$ we have

$$
\int_{X} L \varphi d m=\int_{X} \varphi d m
$$

We conclude that $L^{*} m=m$.

Conversely, assume that $L$ acts on $L^{1}(m)$ and that $L^{*} m=m$. Let $A$ be a special set. Then by the definition of the Perron-Frobenius operator we have

$$
m\left(\chi_{A} f\right)=L^{*} m\left(\chi_{A} f\right)=m\left(L\left(\chi_{A} f\right)\right)=\int_{X} \sum_{T(y)=x} \chi_{A}(y) m(d x)=m(T(A))
$$

Then $m$ is $f$-conformal, proving the theorem.

Remark 11. Note that if we say that $f(y)=e^{-g(y)}$, for a measurable function $g$ then the PerronFroebenius operator becomes

$$
L \varphi(x)=\sum_{T(y)=x} \varphi(y) e^{g(y)}
$$

Which is the usual form this operator in Statistical Mechanics.

Remark 12. Note as well that the theorem applies to $X=\Sigma_{A}$ and $T=\sigma$, the shift map. In this case, since the alphabet is finite and the fact that the cylinders are special sets for $\sigma$ we have a characterization for $f$-conformal measures.

Now, we define a new kind of conformal measure, which the structure of a groupoid is more evident, see [Tho14].

Definition 25. $(G, c)$-conformal with exponent $\beta$

Let $\beta \in \mathbb{R} \backslash\{0\}, G$ a étale locally compact Groupoid and $c: G \mapsto \mathbb{R}$ a continuous homomorphism. A finite Borel measure $m$ on $G^{(0)}$ is $(G, c)$-conformal with exponent $\beta$ when

$$
m(s(W))=\int_{r(W)} e^{\beta c\left(r_{W}^{-1}(x)\right)} d m(x)
$$

for every open bisection $W$ of $G$. 
We shall see throughout this work, that these definitions are actually equivalent, for a certain Groupoid $G$ and a particular continuous homomorphism. For this purpose, we define the notion of quasi-invariant measure, which appears in Renault's Lecture Notes [Ren80].

Definition 26. Let $G$ be a $L C H$ étale groupoid with $G^{(0)}$ the unit space. A Radon measure $\mu$ on $G^{(0)}$ is quasi-invariant if the measures $r^{*} \mu$ and $s^{*} \mu$ on $G$ are equivalent(Have the same null-sets), where $r^{*} \mu$ is the measure on $G$ defined for $f \in C_{c}(G)$ by

$$
\int_{G} f d\left(r^{*} \mu\right)=\int_{G^{(0)}} \sum_{\gamma \in r^{-1}(x)} f(\gamma) d \mu(x)
$$

a similar expression for $s^{*} \mu$, changing $r$ to $s$.

Definition 27. The Radon-Nikodym derivative $D$ of a quasi-invariant measure $\mu$ is the function $D=\frac{d r^{*} \mu}{d s^{*} \mu}$, which makes sense since they are equivalent.

Next lemma shows how these measures are related.

Lemma 5. Let $G$ be an locally compact Hausdorff étale Groupoid. A regular measure $\mu \in G^{(0)}$ is $(G, c)$ - conformal with exponent $\beta$ if and only if it is quasi invariant with Radon-Nikodym derivative $e^{-\beta c}$

Proof. Thoughout this proof we are going to abuse of the following fact from measure-theory. If two finite measures $\mu_{1}$ and $\mu_{2}$ are such that $\mu_{1} \ll \mu_{2}, f=\frac{d \mu_{1}}{d \mu_{2}}$ and $f>0$, then $\mu_{2} \ll \mu_{1}$ and $\frac{d \mu_{2}}{d \mu_{1}}=f^{-1}$.

First, we prove that $(G, c)$-conformal with exponent $\beta$ is equivalent to the following expression, for every open bisection $W$,

$$
\frac{d H_{*} \mu}{d \mu}(x)=e^{\beta c\left(\left.r\right|_{W} ^{-1}(x)\right)} \quad x \in r(W)
$$

Where $H: s(W) \rightarrow r(W)$ is defined by $H(s(x))=r(x)$, which is well defined, since $W$ is an open bisection, indeed it can be written as $\left.s\right|_{W} ^{-1} \circ r$. For the forward direction consider any open set $A \subseteq r(W)$, since $A$ is a subset of $G^{(0)}, r$ and $s$ act as the identity on $A$, so $A$ is an open bisection. Applying the definition of $(G, c)$ - conformal we have

$$
\mu(s(A))=\int_{r(A)} e^{\beta c\left(r||_{W}^{-1}(x)\right)} d \mu(x) \Longrightarrow H_{*} \mu(A)=\int_{A} e^{\beta c\left(\left.r\right|_{W} ^{-1}(x)\right)} d \mu(x)
$$

Since this is true for every open set $A \subseteq r(W)$, we conclude it is true for every measurable set $E \subseteq r(W)$ by outer regularity of $\mu$ and we have the result. The converse is trivial using the definition of Radon-Nikodym derivative.

Now consider $T: r(W) \rightarrow s(W)$ the inverse map of $H$. We show that (3.4) is equivalent to the similar expression

$$
\frac{d T_{*} \mu}{d \mu}(x)=e^{-\beta c\left(\left.s\right|_{W} ^{-1}(x)\right)} \quad x \in s(W)
$$

First the forward direction. Let $B \subseteq s(W), H(B) \subseteq r(W)$, so

$$
\mu(B)=H_{*} \mu(H(B)) \stackrel{3.4}{=} \int_{B} \frac{d H_{*} \mu}{d \mu}(H(y)) d \mu(H(y))=\int_{B} \frac{d H_{*} \mu}{d \mu} \circ H(y) d T_{*} \mu(y)
$$

This equation then shows that, by the definition of Radon-Nikodym derivative,

$$
\frac{d \mu}{d T_{*} \mu}(y)=\frac{d H_{*} \mu}{d \mu} \circ H(y) \Longrightarrow \frac{d T_{*} \mu}{d \mu}(y)=\left(\frac{d H_{*} \mu}{d \mu} \circ H(y)\right)^{-1} y \in s(W)
$$


And this proves that

$$
\frac{d T_{*} \mu}{d \mu}(x)=e^{-\beta c\left(\left.s\right|_{W} ^{-1}(x)\right)} \quad x \in s(W)
$$

The other direction is a similar calculation. Finally we prove that (3.5) is equivalent to $\mu$ being quasi invariant with Radon-Nikodym derivative $e^{-\beta c}$. First suppose that (3.5) is true. Let $W$ be an open bisection and let $f \in C_{c}(G)$ be such that $\operatorname{supp}(f) \subseteq W$. Note that for such functions

$$
\begin{aligned}
\int f d r^{*} \mu & =\int_{r(W)} f\left(\left.r\right|_{W} ^{-1}(x)\right) d \mu(x) \\
\int f d s^{*} \mu & =\int_{s(W)} f\left(\left.s\right|_{W} ^{-1}(x)\right) d \mu(x)
\end{aligned}
$$

Then,

$$
\begin{aligned}
& \int f d r^{*} \mu=\int_{r(W)} f\left(\left.r\right|_{W} ^{-1}(x)\right) d \mu(x)=\int_{s(W)} f\left(\left.r\right|_{W} ^{-1}\left(T^{-1} x\right)\right) d \mu\left(T^{-1}(x)\right)=\int_{s(W)} f\left(\left.s\right|_{W} ^{-1}(x)\right) d T_{*} \mu(x) \\
& \stackrel{3.5}{=} \int_{s(W)} f\left(\left.s\right|_{W} ^{-1}(x)\right) e^{-\beta c\left(\left.s\right|_{W} ^{-1}(x)\right)} d \mu=\int f e^{-\beta c} d s^{*} \mu .
\end{aligned}
$$

We conclude that

$$
\int f d r^{*} \mu=\int f e^{-\beta c} d s^{*} \mu
$$

for every $f \in C_{c}(G)$ with support on an open bisection. By Lemma 3 every $f \in C_{c}(G)$ is in the linear span of functions of the previous type. By the linearity of the integral we conclude that the identity works for every such $f$ and so $\frac{d r^{*} \mu}{d s^{*} \mu}=e^{-\beta c}$.

On the other hand if we have $\frac{d r^{*} \mu}{d s^{*} \mu}=e^{-\beta c}$, in a similar calculation as above we have for any $W \subset G$ open bisection,

$$
\int_{s(W)} f\left(\left.s\right|_{W} ^{-1}(x)\right) e^{-\beta c\left(\left.s\right|_{W} ^{-1}(x)\right)} d \mu=\int_{s(W)} f\left(\left.s\right|_{W} ^{-1}(x)\right) d T_{*} \mu(x),
$$

for every $f \in C_{c}(G)$ such that $\operatorname{supp}(f) \subset W$. We conclude using that $\mathbb{1}_{D}$ for $D \subset s(W)$ can be approximated by functions of the form $\left.f \circ s\right|_{W} ^{-1}$, for $f \in C_{c}(G)$ and $\operatorname{supp}(f) \subset W$, then by the Dominated Convergence Theorem we have $T_{*} \mu(D)=\int_{D} e^{-\beta c\left(\left.s\right|_{W} ^{-1}(x)\right)}$, concluding the lemma.

\subsection{The Renault-Deaconu Groupoid}

We will now define one of the most important objects for this work, the Renault-Deaconu Groupoid. Let $X$ be a locally compact second countable Hausdorff space and $\sigma$ a local homeomorphism on $X^{1}$. We define the set

$$
\mathcal{G}=\left\{(x, k, y) \in X \times \mathbb{Z} \times X: \exists n, m \in \mathbb{N}, k=n-m, \sigma^{n}(x)=\sigma^{m}(y)\right\} .
$$

The set of composable parts is

$$
\mathcal{G}^{(2)}=\left\{\left((x, k, y),\left(x^{\prime}, k^{\prime}, y^{\prime}\right)\right) \in \mathcal{G} \times \mathcal{G}: y=x^{\prime}\right\} .
$$

We define the multiplication and inversion by

\footnotetext{
${ }^{1} \mathrm{An}$ important observation is that $X$, with those hypothesis is a metric space.
} 


$$
(x, k, y)\left(y, k^{\prime}, y^{\prime}\right)=\left(x, k+k^{\prime}, y^{\prime}\right) \quad \text { and } \quad(x, k, y)^{-1}=(y,-k, x) .
$$

For the unit space we have

$$
\mathcal{G}^{(0)}=\{(x, 0, x): x \in X\} .
$$

It is clear that $\mathcal{G}^{(0)} \subseteq \mathcal{G}$ and from now on we identify $\mathcal{G}^{(0)}$ and $X$ by the identification map $x \mapsto$ $(x, 0, x)$. The range and source maps are defined as $r((x, k, y))=(x, 0, x)=x$ and $s((x, k, y))=y$. That all this constitutes a groupoid structure is rather trivial. Now we will put a certain topology on $\mathcal{G}$ with the intention that it becomes a locally compact Hausdorff étale groupoid and our previous results will apply to it. Consider the sets:

$$
\mathcal{U}_{A, B}^{n, m}=\left\{(x, k, y) \in \mathcal{G}: k=n-m, \sigma^{n}(x)=\sigma^{m}(y), x \in A, y \in B\right\}
$$

Where $A$ and $B$ are open sets of $X$ and $n, m \in \mathbb{N}$

Theorem 8. The groupoid of Renault-Deaconu $\mathcal{G}$, with the topology defined by $\mathcal{U}_{A, B}^{n, m}$ is an topological groupoid, LCH étale and second countable.

Proof. The sets of the form $\mathcal{U}_{A, B}^{n, m}$ constitutes a basis for the topology of $\mathcal{G}$. Indeed, let:

$$
(x, k, y) \in \mathcal{U}_{A_{1}, B_{1}}^{n_{1}, m_{1}} \cap \mathcal{U}_{A_{2}, B_{2}}^{n_{2}, m_{2}}
$$

Clearly, $k=n_{i}-m_{i}$ and $\sigma^{n_{i}}(x)=\sigma^{m_{i}}(y)$, for $i=1,2$. Define $p_{1}=n_{2}$ and $p_{2}=n_{1}$. Since $\sigma^{p_{i}}$ is a local homeomorphism, there exists an open neighborhood $U_{i} \subseteq X$ of $\sigma^{n_{i}}(x)$ such that $\sigma^{p_{i}}$ is injective. Now define $\mathcal{U}_{A, B}^{n, m}$ by:

$$
\begin{array}{r}
n=n_{1}+p_{1}, m=m_{1}+p_{1} \\
A=A_{1} \cap A_{2} \cap \sigma^{-n_{1}}\left(U_{1}\right) \cap \sigma^{-n_{2}}\left(U_{2}\right) \\
B=B_{1} \cap B_{2} \cap \sigma^{-m_{1}}\left(U_{1}\right) \cap \sigma^{-m_{2}}\left(U_{2}\right) .
\end{array}
$$

Clearly $n-m=k$ and by construction we have $x \in A$ and $y \in B$. Both are open sets. Finally

$$
\sigma^{n}(x)=\sigma^{n_{1}+p_{1}}(x)=\sigma^{p_{1}}\left(\sigma^{n_{1}}(x)\right)=\sigma^{p_{1}}\left(\sigma^{m_{1}}(y)\right)=\sigma^{m}(y)
$$

Concluding that $(x, k, y) \in \mathcal{U}_{A, B}^{n, m}$. Now we just need to verify that $\mathcal{U}_{A, B}^{n, m} \subseteq \mathcal{U}_{A_{1}, B_{1}}^{n_{1}, m_{1}} \cap \mathcal{U}_{A_{2}, B_{2}}^{n_{2}, m_{2}}$. Now let $\left(x^{\prime}, k, y^{\prime}\right) \in \mathcal{U}_{A, B}^{n, m}$. For $\mathrm{i}=1,2$, we have:

$$
\sigma^{p_{i}}\left(\sigma^{n_{i}}\left(x^{\prime}\right)\right)=\sigma^{n}\left(x^{\prime}\right)=\sigma^{m}\left(y^{\prime}\right)=\sigma^{m_{i}+p_{i}}\left(y^{\prime}\right)=\sigma^{p_{i}}\left(\sigma^{m_{i}}\left(y^{\prime}\right)\right)
$$

Now by injectivity of $\sigma^{p_{i}}$ in $U_{i}$ and the fact that $x^{\prime} \in A \subseteq \sigma^{-n_{i}}\left(U_{i}\right), y^{\prime} \in B \subseteq \sigma^{-m_{i}}\left(U_{i}\right)$, we have $\sigma^{n_{i}}\left(x^{\prime}\right)=\sigma^{m_{i}}\left(y^{\prime}\right)$, concluding that $\left(x^{\prime}, k, y^{\prime}\right) \in \mathcal{U}_{A_{1}, B_{1}}^{n_{1}, m_{1}} \cap \mathcal{U}_{A_{2}, B_{2}}^{n_{2}, m_{2}}$.

Now let us prove that it is a topological groupoid, i.e, the inverse and composition maps are continuous. Consider the inverse $I: \mathcal{G} \rightarrow \mathcal{G},(x, k, y) \mapsto\left(y, k^{-1}, x\right)$. Let $\lambda=(x, k, y) \in \mathcal{G}$ and a convergent net $\lambda_{i}=\left(x_{i}, k_{i}, y_{i}\right) \rightarrow \lambda$. There exists $\lambda \in \mathcal{U}_{A, B}^{n, m}$ for certain $n, m, A, B$, since it constitutes a basis. There exists a index $i_{0}$ such that $\lambda_{i} \in \mathcal{U}_{A, B}^{n, m}$ for all $i>i_{0}$. It is then clear that $I\left(\lambda_{i}\right), I(\lambda) \in$ $\mathcal{U}_{B, A}^{m, n}$ which proves the continuity of the map $I$. Now, let $c: \mathcal{G}^{(2)} \rightarrow \mathcal{G},((x, k, y),(y, h, z)) \mapsto$ $(x, k h, z)$, the composition map. Let $\eta=((x, k, y),(y, h, z)) \in \mathcal{G}^{(2)}$ and a convergent net $\eta_{i}=$ $\left(\left(x_{i}, k_{i}, y_{i}\right),\left(y_{i}, h_{i}, z_{i}\right)\right) \rightarrow \eta$. We remember that $\mathcal{G}^{(2)}$ is given the product topology, so $\left(x_{i}, k_{i}, y_{i}\right) \rightarrow$ $(x, k, y)$ and $\left(y_{i}, h_{i}, z_{i}\right) \rightarrow(y, h, z)$. Now, there exists $n, m, j, l \in \mathbb{N}$ and open sets $A, B, C$ of $X$ such that $(x, k, y) \in \mathcal{U}_{A, B}^{n, m}$ and $(y, h, z) \in \mathcal{U}_{B, C}^{j, l}$. It follows that $\left(x_{i}, k_{i}, y_{i}\right) \in \mathcal{U}_{A, B}^{n, m}$ and $\left(y_{i}, h_{i}, z_{i}\right) \in \mathcal{U}_{B, C}^{j, l}$, for all $i>i_{0}$. Observe that $k+h=(n+j)-(m+l)$, then:

$$
c\left(\left(x_{i}, k_{i}, y_{i}\right),\left(y_{i}, h_{i}, z_{i}\right)\right)=\left(x_{i}, k_{i}+h_{i}, z_{i}\right) \in \mathcal{U}_{A, C}^{n+j, m+l}
$$




$$
c((x, k, y),(y, h, z))=(x, k+h, z) \in \mathcal{U}_{A, C}^{n+j, m+l}
$$

Which guarantees the continuity of $c$. Now it is needed to prove that such topology is Hausdorff and locally compact. For Hausdorff, consider $\xi=(x, k, y), \eta=\left(x^{\prime}, h, y^{\prime}\right) \in \mathcal{G}$. As always, $\xi \in \mathcal{U}_{A, B}^{n, m}:=V_{\xi}$ and $\eta \in \mathcal{U}_{A^{\prime}, B^{\prime}}^{j, l}:=V_{\eta}$. If $x \neq x^{\prime}$, there exists two disjoint open neighborhoods of $x$ and $x^{\prime}$. When taking $A$ and $A^{\prime}$ above we could have made them subsets of the neighborhoods and then we would conclude that $V_{\xi} \cap V_{\eta}=\emptyset$. If we have $y \neq y^{\prime}$, we could have done a similar thing. Finally let, $x=x^{\prime}$, $y=y^{\prime}$ and $k \neq h$, clearly $V_{\xi}$ and $V_{\eta}$ are disjoint. $\mathcal{G}$ is then Hausdorff.

For the proof of locally compact, consider for $n, m \in \mathbb{N}$ :

$$
R(n, m):=\left\{(x, y) \in X \times X \mid \sigma^{n}(x)=\sigma^{m}(y)\right\}
$$

and the function $i: R(n, m) \rightarrow \mathcal{G},(x, y) \mapsto(x, n-m, y)$. Let us show that $i$ is continuous and $R(n, m)$ is a locally compact subspace on the product topology of $X \times X$. For the second, consider a net $\xi_{i}=\left(x_{i}, y_{i}\right)$ in $R(n, m)$ which converges to $\xi=(x, y) \in X \times X \Longrightarrow x_{i} \rightarrow x, y_{i} \rightarrow y$, and by continuity $\sigma^{n}\left(x_{i}\right) \rightarrow \sigma^{n}(x)$ and $\sigma^{m}\left(y_{i}\right) \rightarrow \sigma^{m}(y)$. We have $\sigma^{n}\left(x_{i}\right)=\sigma^{m}\left(y_{i}\right)$, and so we have $\sigma^{n}(x)=\sigma^{m}(y)$. We have then proved that $\xi \in R(n, m)$, i.e, $R(n, m)$ is a closed set of $X \times X$, therefore a locally compact in the subspace topology.

We now need to prove that $i$ is a continuous map.

Given $\left(x_{0}, y_{0}\right) \in R(n, m)$ and $V^{\prime}$ an open neighborhood of $\left(x_{0}, n-m, y_{0}\right)$, which is the image of $\left(x_{0}, y_{0}\right)$ by $i$. There are $k, l, A, B$ such that:

$$
\left(x_{0}, n-m, y_{0}\right) \in \mathcal{U}_{A, B}^{k, l} \subseteq V^{\prime}
$$

Clearly we have $n-m=k-l \Longrightarrow(n-k)=(m-l)$ and $\sigma^{k}\left(x_{0}\right)=\sigma^{l}\left(y_{0}\right)$. There exists an open neighborhood $V$ of $\sigma^{k}\left(x_{0}\right)$ such that $\sigma^{m}$ is injective (As always it was used local homeomorphism). Define:

$$
\begin{gathered}
W:=B \cap \sigma^{-l}(V) \\
U:=A \cap \sigma^{-k}(V) \\
Z:=(U \times V) \cap R(n, m)
\end{gathered}
$$

We have that $\left(x_{0}, y_{0}\right) \in Z$, and $Z$ is an open set. We should now be able to prove that $i(Z) \subseteq V^{\prime}$ and conclude that $i$ is continuous. Let $(x, y) \in Z$, therefore $i((x, y))=(x, n-m, y)$, we remember that $n-m=k-l$. Clearly $x \in A$ and $y \in B$. Now:

$$
\sigma^{m}\left(\sigma^{k}(x)\right)=\sigma^{m+k}=\sigma^{n+l}(x)=\sigma^{m}\left(\sigma^{l}(y)\right)
$$

By the definition of $U, V$, we have that $\sigma^{k}(x), \sigma^{l}(y) \in V$. Therefore, since $\sigma^{m}$ is injective in such set we conclude that:

$$
\sigma^{k}(x)=\sigma^{l}(y) \Longrightarrow i((x, y)) \in \mathcal{U}_{A, B}^{k, l} \subseteq V^{\prime} \Longrightarrow i(Z) \subseteq V^{\prime}
$$

This proves the continuity of $i$. We restrict $i$ onto its image, i.e, $i: R(n, m) \rightarrow \mathcal{U}_{X, X}^{n, m}$, the continuity of the inverse has a similar proof as the continuity of $i$, then $i$ is not only continuous but a open map. Given $\xi \in \mathcal{G}$, there exists $n, m, A, B$ s.t $\xi \in \mathcal{U}_{A, B}^{n, m} \subseteq \mathcal{U}_{X, X}^{n, m}$. Which means there is $\lambda \in R(n, m)$ such that $i(\lambda)=\xi$. By local compactness of $R(n, m)$ there is a open set $V$ and a compact set $K$ such that $\lambda \in V \subset K$. By continuity $i(K)$ is compact, by it being a open map and $\mathcal{U}_{X, X}^{n, m}$ being open on $\mathcal{G}, i(V)$ is open on $\mathcal{G}$. Then $\xi \in i(V) \subset i(K)$, concluding that $\mathcal{G}$ is locally compact.

Finally we prove that $\mathcal{G}$ is an étale groupoid. Since $r$ and $s$ have analogous proofs, we just prove that $r$ is a local homeomorphism. We remember that given $\xi=(x, k, y) \Longrightarrow r(\xi)=x$. Now there exists $n, m, A, B$ such that $\xi \in \mathcal{U}_{A, B}^{n, m}, k=n-m$ and $\sigma^{n}(x)=\sigma^{m}(y)$. Now, since $\sigma^{n}$ and $\sigma^{m}$ are local homeomorphisms, there are open neighborhoods $U \subseteq A$ and $V \subseteq B$ of $x$ and $y$ such that $\left.\sigma^{n}\right|_{U}$ 
and $\left.\sigma^{m}\right|_{V}$ are homeomorphisms.

Let $Y:=\mathcal{U}_{U, V}^{n, m}$. It is clear that $\xi$ is in $Y$. We show that $\left.r\right|_{Y}$ is injective. Given two point $\left(x_{1}, k, y_{1}\right),\left(x_{2}, k, y_{2}\right) \in Y$ such that their range are the same (it means $\left.x_{1}=y_{1}\right)$, then $\sigma^{n}\left(x_{1}\right)=$ $\sigma^{n}\left(x_{2}\right) \Longrightarrow \sigma^{m}\left(y_{1}\right)=\sigma^{m}\left(y_{2}\right)$. The map is one to one in $V$, then $y_{1}=y_{2}$ and $\left.r\right|_{Y}$ is injective and $Y$ is an open set. Since it is a topological groupoid $r$ is continuous, therefore we just need to prove that the inverse is continuous and $r(Y)$ is an open set. For the inverse, it is the map:

$$
\begin{gathered}
u: r(Y)=\{z \in U \mid \quad(z, k, h) \in Y\} \rightarrow Y \\
z \mapsto(z, k, h)
\end{gathered}
$$

Here $h$ is the unique element of $V$ such that $\sigma^{m}(h)=\sigma^{n}(z)$. Consider $z_{i} \rightarrow z$ on $r(Y)$. We need that $u\left(z_{i}\right) \rightarrow u(z)$, i.e, $\left(z_{i}, k, h_{i}\right) \rightarrow(z, k, h)$. As $u\left(z_{i}\right)$ and $u(z)$ are in $Y$, we have that $\sigma^{n}\left(z_{i}\right)=$ $\sigma^{m}\left(h_{i}\right)$ and $\sigma^{n}(z)=\sigma^{m}(h)$. Then we have $\sigma^{n}\left(z_{i}\right) \rightarrow \sigma^{n}(z) \Longrightarrow \sigma^{m}\left(h_{i}\right) \rightarrow \sigma^{m}(h)$. Now since $h_{i}, h \in V$ and $\sigma^{m}$ is an homeomorphism on $V, \sigma^{-m}$ is the inverse and it is continuous $\Longrightarrow h_{i} \rightarrow h$. The later implies that $u\left(z_{i}\right) \rightarrow u(z)$.

Finally we show that $r(Y)$ is open, which concludes the proposition. Let $z \in r(Y)$ and a convergent net $z_{i} \rightarrow z$. We need to show that $z_{i} \in r(Y)$ for large $i$, (it is an interior point). We remember that $\left.\sigma^{n}\right|_{U}$ and $\left.\sigma^{m}\right|_{V}$ are homeomorphisms, defining $H:=\sigma^{n}(U) \cap \sigma^{m}(V)$, it is non empty because $\xi$ is there. As $z \in r(Y), z \in U$, and it being an open set $z_{i} \in U$ for large enough $i$ and $\sigma^{n}\left(z_{i}\right) \rightarrow \sigma^{n}(z)=\sigma^{m}(h)$.

Now, $\left(\left.\sigma^{m}\right|_{H}\right)^{-1}\left(\sigma^{n}\left(z_{i}\right)\right) \rightarrow\left(\left.\sigma^{m}\right|_{H}\right)^{-1}\left(\sigma^{m}(h)\right)=h$ and $\left(z_{i}, k,\left(\left.\sigma^{m}\right|_{H}\right)^{-1}\left(\sigma^{n}\left(z_{i}\right)\right)\right) \in Y \Longrightarrow z_{i} \in$ $r(Y)$, which concludes that $r(Y)$ is open, therefore $r$ is a local homeomorphism and it is done.

Although not explicit in the computations above, the second countability of $\mathcal{G}$ comes from the fact that we could have used basic open sets of $X$ on the definition of $\mathcal{U}_{A, B}^{n, m}$, which does not change the previous proof and it is clear that this basis is countable.

Now we want to define a continuous homomorphism that will be interesting for our groupoid $\mathcal{G}$. Let $F: X \rightarrow \mathbb{R}$ be a continuous function and define $c_{F}: G \rightarrow \mathbb{R}$, the continuous cocycle associated to $F$, as

$$
c_{F}(x, k, y)=\lim _{n \rightarrow \infty}\left(\sum_{i=0}^{n+k} F\left(\sigma^{i}(x)\right)-\sum_{i=0}^{n} F\left(\sigma^{i}(y)\right)\right)
$$

We don't need to worry about the limit, because for all $(x, k, y) \in \mathcal{G}$ there are $n, m \in \mathbb{N}$ s.t $k=n-m$ and $\sigma^{n}(x)=\sigma^{m}(y)$, and with that we can verify that,

$$
c_{F}(x, k, y)=\sum_{i=0}^{n-1} F\left(\sigma^{i}(x)\right)-\sum_{i=0}^{m-1} F\left(\sigma^{i}(y)\right) .
$$

This means that the limit in the definition is, in fact, always a finite sum.

Proposition 22. $c_{F}$ is a continuous homomorphism.

Proof. Consider $(x, k, y),(y, l, z) \in G$. Let $k=n-m$ and $l=o-p$, such that $\sigma^{n}(x)=\sigma^{m}(y)$ and $\sigma^{o}(y)=\sigma^{p}(z)$.

$$
\begin{aligned}
c_{F}[(x, k, y)(y, l, z)] & =c_{F}[(x, k+l, z)]=\sum_{i=0}^{n+o-1} F\left(\sigma^{i}(x)\right)-\sum_{i=0}^{m+p-1} F\left(\sigma^{i}(z)\right) \\
& =c_{F}(x, k, y)+\sum_{i=0}^{m-1} F\left(\sigma^{i}(y)\right)-\sum_{i=0}^{o-1} F\left(\sigma^{i}(y)\right)+\sum_{i=n}^{n+o-1} F\left(\sigma^{i}(x)\right)-\sum_{i=p}^{m+p-1} F\left(\sigma^{i}(z)\right)+c_{F}(y, l, z) \\
& =c_{F}(x, k, y)+c_{F}(y, l, z)
\end{aligned}
$$



$\mathcal{U}_{W, V}^{n, m}$

For the continuity let $\left(x_{l}, k_{l}, y_{l}\right) \rightarrow(x, k, y)$ and $(x, k, y) \in \mathcal{U}_{W, V}^{n, m}$. For large enough $l,\left(x_{l}, k_{l}, y_{l}\right) \in$

$$
\begin{aligned}
c_{F}(x, k, y) & =\sum_{i=0}^{n-1} F\left(\sigma^{i}(x)\right)-\sum_{i=0}^{m-1} F\left(\sigma^{i}(y)\right)=\sum_{i=0}^{n-1} F\left(\lim _{l} \sigma^{i}\left(x_{l}\right)\right)-\sum_{i=0}^{m-1} F\left(\lim _{l} \sigma^{i}\left(y_{l}\right)\right) \\
& =\lim _{l} \sum_{i=0}^{n-1} F\left(\sigma^{i}\left(x_{l}\right)\right)-\sum_{i=0}^{m-1} F\left(\sigma^{i}\left(y_{l}\right)\right)=\lim _{l} c_{F}\left(x_{l}, k_{l}, y_{l}\right)
\end{aligned}
$$

The following sets are going to be useful in the next lemma. Fix $k \in \mathbb{Z}$, for each $n \in \mathbb{N}$ such that $n+k \geq 0$, take

$$
\mathcal{G}(k, n)=\left\{(x, l, y) \in X \times \mathbb{Z} \times X: l=k, \sigma^{k+n}(x)=\sigma^{n}(y)\right\}
$$

We observe that $\mathcal{G}(k, n) \subseteq \mathcal{G}(k, n+1)$.

Define as well:

$$
\mathcal{G}(k)=\bigcup_{n \geq-k, n \in \mathbb{N}} \mathcal{G}(k, n)
$$

Note that $\mathcal{G}=\bigsqcup_{k \in \mathbb{Z}} \mathcal{G}(k)$.

This next lemma show us that our definition in the beginning of Chapter 3, is equivalent to definition 25 , what we mentioned before.

Lemma 6. Let $m$ be a regular Borel measure on $X$ and $\mathcal{G}$ the Renault-Deaconu Groupoid. Then $m$ is $\left(\mathcal{G}, c_{F}\right)$ - conformal with exponent $\beta$ if and only if $m$ is $e^{\beta F}-$ conformal.

Proof. Assume that $m$ is $\left(\mathcal{G}, c_{F}\right)-$ conformal with exponent $\beta$ and consider a Borel subset $A \subseteq X$ such that $\sigma$ is injective on $A$. Since $\sigma$ is a local homeomorphism and $X$ is second countable we can write $A$ as a countable disjoint union

$$
A=\bigsqcup_{i \in I} A_{i}
$$

of Borel sets with the property that for each $i$ there is an open set $U_{i}$ such that $A_{i} \subseteq U_{i}$ and $\sigma: U_{i} \rightarrow \sigma\left(U_{i}\right)$ is a homeomorphism. To prove that, see that for every $x \in A$ there is a basic set $B_{i_{x}}$ such that $\left.\sigma\right|_{B_{i_{x}}}$ is a homeomorphism. $\left\{B_{i_{x}}: x \in A\right\}=\left\{B_{q_{n}}: n \in \mathbb{N}\right\}$ since the first is countable. Let $C_{i}=B_{q_{i}} \cap A$, so $A=\bigcup_{i \in \mathbb{N}} C_{i}$. Defining $A_{i}=C_{i} \backslash \bigcup_{n=1}^{i-1} C_{n}$ we conclude what was stated.

For each $i$ and each open subset $V \subseteq U_{i}$ the set $W=\{(x, 1, \sigma(x)): x \in V\}$ is open, $\left.r\right|_{W}$ is injective since if $r(x, 1, \sigma(x))=r(z, 1, \sigma(z)) \Longrightarrow x=z$, but then $\sigma(x)=\sigma(z)$, the same can be done for $s$, noting that $\sigma$ is injective on $V$. The set $W$ is then a open bisection in $\mathcal{G}$ with $s(W)=\sigma(V), r(W)=V$, and therefore we have:

$$
m(\sigma(V))=\int_{V} e^{\beta c_{F}(x, 1, \sigma(x))} d m(x)=\int_{V} e^{\beta F(x)} d m(x)
$$

By additivity and regularity of $m$,

$$
m(\sigma(A))=\sum_{i \in I} m\left(\sigma\left(A_{i}\right)\right)=\sum_{i} \int_{A_{i}} e^{\beta F(x)} d m(x)=\int_{A} e^{\beta F(x)} d m(x)
$$

Assume next that $m$ is $e^{\beta F}-$ conformal. If $A \subseteq X$ is such that $\left.\sigma^{n}\right|_{A}$ is injective then clearly 
$\left.\sigma^{p}\right|_{A}$ is injective for all $1 \leq p \leq n$. We shall prove the expression:

$$
m\left(\sigma^{n}(A)\right)=\int_{A} e^{\beta \sum_{i=0}^{n-1} F\left(\sigma^{i}(x)\right)} d m(x)
$$

For that consider $\mathbb{B}(X)$, the Borel sigma algebra of $X$ and let $A$ be the set we said above. Then for each $1 \leq i \leq n$ we can define the measures $m \circ \sigma^{i}(E):=m\left(\sigma^{i}(E)\right)$ for $E \in \mathbb{B}(X) \cap A$. One needs to ask if $\sigma^{i}(E) \in \mathbb{B}(X)$, for that we use two non-trivial results. First we use that every locally compact second contable Hausdorff space is a Polish space. Second we use Lusin-Souslin theorem (it can be found in [Kec95] page 89), the fact that $\sigma^{i}$ is continuous and $\left.\sigma^{i}\right|_{E}$ is injective. To verify the axioms of measure is not difficult. Observe that $m \circ \sigma^{i+1} \ll m \circ \sigma^{i}$ for $i=0, \ldots, n-1$. We can see as well that:

$$
m \circ \sigma^{i+1}(E)=m\left(\sigma\left(\sigma^{i}(E)\right)\right)=\int_{\sigma^{i}(E)} e^{\beta F(x)} d m(x)=\int_{E} e^{\beta F\left(\sigma^{i}(x)\right)} d m \circ \sigma^{i}(x)
$$

We can then have the Radon-Nikodym derivative:

$$
\frac{d m \circ \sigma^{i+1}}{d m \circ \sigma^{i}}=e^{\beta F\left(\left.\sigma^{i}\right|_{A}\right)}
$$

With this it is not difficult to arrive at equation (3.6). Now let $B$ be a Borel subset of $X$ such that $\sigma^{m}$ is injective on $B$ and $\sigma^{n}(A)=\sigma^{m}(B)$. Consider $\sigma^{j}: \sigma^{m-j}(B) \rightarrow \sigma^{m}(B)=\sigma^{n}(A)$, for $j=$ $1, \ldots, m$. These functions are injective in such domains, since if $\sigma^{j}(x)=\sigma^{j}(y) \Longrightarrow \sigma^{j}\left(\sigma^{m-j}\left(z_{1}\right)\right)=$ $\sigma^{j}\left(\sigma^{m-j}\left(z_{2}\right)\right) \Longrightarrow z_{1}=z_{2} \Longrightarrow x=y$.

Now consider the measures $m \circ \sigma^{-j}(E):=m\left(\sigma^{-j}(E)\right)$, for $E \in \mathbb{B}(X) \cap \sigma^{n}(A), j=1, \ldots, m$, then

$$
m \circ \sigma^{-l+1}(E)=\int_{\sigma^{-l}(E)} e^{\beta F(x)} d m(x)
$$

So $m \circ \sigma^{-l+1} \ll m \circ \sigma^{-l}, l=1, \ldots, m$. We have as well that

$$
\frac{d m \circ \sigma^{-l}}{d m \circ \sigma^{-l+1}}(x)=e^{-\beta F\left(\sigma^{-l}(x)\right)}
$$

With that we have

$$
\begin{gathered}
m(B)=m\left(\sigma^{-m}\left(\sigma^{n}(A)\right)\right)=m \circ \sigma^{-m}\left(\sigma^{n}(A)\right)=\int_{\sigma^{n}(A)} d m \circ \sigma^{-m}(x)= \\
\int_{\sigma^{n}(A)} \frac{d m \circ \sigma^{-m}}{d m \circ \sigma^{-m+1}}(x) d m \circ \sigma^{-m+1}(x)=\ldots=\int_{\sigma^{n}(A)} e^{-\beta\left(\sum_{j=1}^{m} F\left(\sigma^{-j}(x)\right)\right)} d m(x)
\end{gathered}
$$

Finally we have get to the identity:

$$
m(B)=\int_{A} e^{\beta\left(\sum_{i=0}^{n-1} F\left(\sigma^{i}(x)\right)-\sum_{j=1}^{m} F\left(\sigma^{-j}\left(\sigma^{n}(x)\right)\right)\right)} d m(x)
$$

Now consider a open bisection $W$ of $\mathcal{G}$. For $k \in \mathbb{Z}$ we set $W_{k}=\mathcal{G}(k) \cap W$. By the $\sigma$-additivity of the measure $m$, we just need to look at those set to establish (25), we can reduce it once more if we consider sets $W(k, n)=W \cap \mathcal{G}(k, n) . \sigma^{n+k}$ is injective on $r(W(k, n))$ and $\sigma^{n}$ is injective on $s(W(k, n))$. We have as well that $\sigma^{n+k}(r(W(k, n)))=\sigma^{n}(s(W(k, n)))$. We can then use the identity (3.7) and get:

$$
m(s(W(k, n)))=\int_{r(W(k, n))} e^{\beta\left(\sum_{i=0}^{n+k-1} F\left(\sigma^{i}(x)\right)-\sum_{j=1}^{m} F\left(\sigma^{-j}\left(\sigma^{n+k}(x)\right)\right)\right)}
$$


on the other hand:

$$
\begin{aligned}
c_{F}\left(r_{W(k, n)}^{-1}(x)\right) & =\sum_{i=0}^{n+k-1} F\left(\sigma^{i}(x)\right)-\sum_{i=0}^{n-1} F\left(\sigma^{i}(y)\right) \\
& =\sum_{i=0}^{n+k-1} F\left(\sigma^{i}(x)\right)-\sum_{i=1}^{n} F\left(\sigma^{-j}\left(\sigma^{n+k}(x)\right)\right)
\end{aligned}
$$

and we conclude that $m$ is $\left(\mathcal{G}, c_{F}\right)$ - conformal with exponent $\beta$.

Now let us prove a result about a certain decomposition of a measure.

\section{Definition 28. Non-atomic and purely atomic measure}

Let $G$ be a LCH étale groupoid. A finite Borel measure $m$ on $G^{(0)}$ is non-atomic when $m(\{x\})=$ $0 \quad \forall x \in G^{(0)}$

It is called Purely atomic when there is a borel set $A \subseteq G^{(0)}$ such that $m(A)=m\left(G^{(0)}\right)$ and $m(\{a\})>0 \quad \forall a \in A$

Let $m$ be a finite Borel measure on $G^{(0)}$, by corollary 2.6 of [Joh70] we have a decomposition $m=m^{c}+m^{a}$ where $m^{c}$ is non-atomic measure and $m^{a}$ is a purely atomic measure.

Lemma 7. $m$ is $(G, c)$ - conformal with exponent $\beta$ if and only if $m^{c}$ and $m^{a}$ both are.

Proof. Let's assume first that $m$ is $(G, c)-$ conformal with exponent $\beta$. Take $W \subseteq G$ a open bi-section. When $V$ is a open subset of $r(W), r_{W}^{-1}(V)$ is a open bisection since $r_{W}^{-1}(V) \subseteq W$. It follows that $m\left(s\left(r_{W}^{-1}(V)\right)\right)=\int_{V} e^{\beta c\left(r_{W}^{-1}(x)\right)} d m(x)$ for every open subset $V \subseteq r(W)$.

The measures on $r(W)$ given by $B \mapsto m\left(s\left(r_{W}^{-1}(B)\right)\right)$ and $B \mapsto \int_{B} e^{\beta c\left(r_{W}^{-1}(x)\right)} d m(x)$ are outer regular on $r(W)$ so it follows that

$$
m\left(s\left(r_{W}^{-1}(B)\right)\right)=\int_{B} e^{\beta c\left(r_{W}^{-1}(x)\right)} d m(x)
$$

for every borel $B \subseteq r(W)$. Let $E$ be the set of atoms for $m$, i.e the points that have positive measure. Since $G$ is covered by bi-sections, we have by (3.8) that $s\left(r^{-1}(E)\right)=E=r\left(s^{-1}(E)\right)$; puting back into (3.8) we have

$$
\begin{aligned}
& m^{c}(s(W))=m(s(W) \backslash E)=m\left(s\left(r_{W}^{-1}(r(W) \backslash E)\right)\right) \\
& =\int_{r(W) \backslash E} e^{\beta c\left(r_{W}^{-1}(x)\right)} d m(x)=\int_{r(W)} e^{\beta c\left(r_{W}^{-1}(x)\right)} d m^{c}(x)
\end{aligned}
$$

Similarly $m^{a}(s(W))=\int_{r(W)} e^{\beta c\left(r_{W}^{-1}(x)\right)} d m^{a}(x)$ and we conclude that $m^{c}$ and $m^{a}$ are $(G, c)-$ conformal with exponent $\beta$. The converse is simple.

\subsection{The Dinamics and KMS states}

We now wish to define a one-parameter group of automorphisms on the $C_{r}^{*}(\mathcal{G})$, which, in the case of the Renault-Deaconu groupoid, is isomorphic to $C^{*}(\mathcal{G})$. Again, in this section $\mathcal{G}$ is the Renault-Deaconu Groupoid. This section has as reference [Ren09].

For each $t \in \mathbb{R}$ we define the map $\tau_{t}: C_{c}(\mathcal{G}) \rightarrow C_{c}(\mathcal{G})$ by $\tau_{t}(f)(\gamma)=e^{i t c_{F}(\gamma)} f(\gamma)$, for $f \in C_{c}(\mathcal{G})$ and $\gamma \in \mathcal{G}$. One needs to verify that $\tau_{t} \in \operatorname{Aut}\left(C_{c}(\mathcal{G})\right)$ and that $\tau$ is a one-parameter group of the *-algebra $C_{c}(\mathcal{G})$. 
Proposition 23. The one-parameter group $\tau$ above extends to a strongly continuous one-parameter automorphism group of $C_{r}^{*}(\mathcal{G})$.

Proof. Let $x \in \mathcal{G}^{(0)}$ and let $\pi_{\lambda}^{x}$ be the regular representation of $C_{c}(G)$ on $\ell^{2}\left(G_{x}\right)$, which we introduced in chapter 2. For all $f \in C_{c}(\mathcal{G})$, we can deduce the equality $\pi_{\lambda}^{x} \circ \tau_{t}(f)=V \pi_{\lambda}^{x}(f) V^{*}$ where $V$ is the unitary operator on $\ell^{2}\left(\mathcal{G}_{x}\right)$ given by $V \zeta(\gamma)=e^{i t c_{F}(\gamma)} \zeta(\gamma)$. With that in mind, we have that $\left\|\tau_{t}(f)\right\|_{r}=\|f\|_{r}$, which shows that $\tau$ extends to a one-parameter automorphism group of $C_{r}^{*}(\mathcal{G})$. For $f \in C_{c}(\mathcal{G})$, it is clear that $t \mapsto \tau_{t}(f)$ is continuous, since it is basically a multiplication. By density of $C_{c}(\mathcal{G})$ in $C^{*}(\mathcal{G})$ we have that $\tau$ is strongly continuous.

Our objective now is to prove that a $e^{\beta F}$-conformal measure $m$ has a state $\phi_{m}$ associated with it which is $K M S_{\beta}$ under the the dynamics $\tau$

Proposition 24. The elements of the $*$-algebra $C_{c}(\mathcal{G})$ are entire analytic for $\tau$.

Proof. The linear operator $\delta$ on the domain $D(\delta)=C_{c}(G)$ given by $\delta f(x)=i c_{F}(x) f(x)$ is the restriction of the generator of $\tau$ on $C_{c}(\mathcal{G})$. To prove that, we must first remember that representations on $C_{c}(\mathcal{G})$ are bounded by the I-norm, defined in Chapter 3 . Then, for any continuous function $\phi$ on $\mathcal{G}$ and $f \in C_{c}(\mathcal{G})$,

$$
\|\phi f\|_{r} \leq\|\phi f\|_{I} \leq \sup _{x \in K}|\phi(x)|\|f\|_{I} .
$$

Where $K$ is the support of $f$. Using the above inequality Then we compute,

$$
\left\|\frac{\tau_{t} f-f}{t}-\delta f\right\|_{r} \leq \sup _{K}\left|\frac{e^{i t c_{F}(x)}-1}{t}-i c_{F}(x)\right|\|f\|_{I}
$$

Letting $t \rightarrow 0^{+}$, the right hand side goes to zero and, indeed $\delta$ is the restriction of the generator of $\tau$. Now, $\delta^{n}$ exists for every $n$ and we observe that $\delta^{n} f(x)=\left(i c_{F}(x)\right)^{n} f(x)$ and using again the inequality above

$$
\left.\left\|\delta^{n} f\right\|_{r} \leq \sup _{K}\left|c_{F}(x)\right|\right)^{n}\|f\|_{I}
$$

Therefore $\sum_{n=0}^{\infty} \frac{t^{n}}{n !}\left\|\delta^{n} f\right\|_{r}<\infty$ for all $t \in \mathbb{R}$. This proves that $f \in C_{c}(\mathcal{G})$ is entire analytic for $\tau$.

Theorem 9. With the Renault-Deaconu Groupoid $\mathcal{G}$, the continuous homomorphism $c_{F}$ and the associated automorphism group $\tau$. Let $\beta \in \mathbb{R}$.

$A e^{\beta F}-$ conformal Borel probability measure $m$ on $X \sim \mathcal{G}^{(0)}$ gives rise to a $K M S_{\beta}$ state $\phi_{m}$ for $\tau$ such that

$$
\phi_{m}(f)=\int_{X} f(x, 0, x) d m(x)
$$

when $f \in C_{c}(G)$. In fact, using the conditional expectation we defined in chapter 2, we can write $\phi_{m}(f)=\int_{X} P(f) d m(x)$ for $f \in C_{c}(G)$.

Proof. First, we observe that $\phi_{m}$ is a state. It is clear that it is positive linear functional. Now,

$$
\left\|\phi_{m}(f)\right\| \leq\|f\|_{\infty} m(X) \leq\|f\|_{r}
$$

So $\phi_{m}$ is continuous, bounded by 1 on a dense subset of $C_{r}^{*}(\mathcal{G})$, it extends continuously by density.

$$
\begin{aligned}
1=m(X) & =\sup \left\{\int_{X} f(x) d m(x) \mid 0 \leq f \leq 1, f \in C_{c}\left(\mathcal{G}^{(0)}\right)\right\} \\
& =\sup \left\{\phi_{m}(f) \mid 0 \leq f \leq 1, f \in C_{c}\left(\mathcal{G}^{(0)}\right)\right\} \leq\left\|\phi_{m}\right\|
\end{aligned}
$$

By the above, $\phi_{m}$ is a state. 
For $f, g \in C_{c}(\mathcal{G})$ the KMS condition says that

$$
\int H e^{-\beta c} d\left(s^{*} m\right)=\int H d\left(r^{*} m\right)
$$

where $H(\gamma)=f\left(\gamma^{-1}\right) g(\gamma)$. Indeed,

$$
\begin{gathered}
\phi_{m}(g f)=\int_{X} g f(x) d m(x)=\int \sum_{h \in r^{-1}(x)} g(h) f\left(h^{-1} x\right)=\int \sum_{h \in r^{-1}(x)} g(h) f\left(h^{-1}\right) \\
=\int \sum_{h \in r^{-1}(x)} H(h) d m(x)=\int H d\left(r^{*} m\right) \\
\phi_{m}\left(f \tau_{i \beta}(g)\right)=\int \sum_{h \in s^{-1}(x)} f\left(x h^{-1}\right) \tau_{i \beta}(g)(h)=\int \sum_{h \in s^{-1}(x)} f\left(h^{-1}\right) e^{-\beta c_{F}(h)} g(h) \\
=\int \sum_{h \in s^{-1}(x)} H(h) e^{-\beta c_{F}(h)}=\int H e^{-\beta c_{F}} d\left(s^{*} m\right)
\end{gathered}
$$

It is clear on the other hand that if $m$ is quasi-invariant with Radon-Nikodym derivative $e^{-\beta c_{F}}$ the above equality is satisfied for every $H \in C_{c}(G)$, by definition. We conclude that $\phi_{m}$ is a $K M S_{\beta}$ state of $C_{r}^{*}(G)$ if $m$ is quasi-invariant with Radon-Nikodym derivative $e^{-\beta c_{F}}$, but by lemma 5 and $6 m$ is $e^{\beta F}-$ conformal Borel probability measure.

\subsection{Cuntz-Krieger Algebras and Groupoids}

First, fix a $n \times n$ matrix $A$ and consider the Shift space $\Sigma_{A}$ as in the chapter Preliminaries. Now let $\ell^{2}\left(\Sigma_{A}\right)$ be the Hilbert space whose elements are functions $f: \Sigma_{A} \rightarrow \mathbb{C}$ such that $\sum_{x \in \Sigma_{A}}|f(x)|<\infty$. There is a canonical orthonormal basis $\ell^{2}\left(\Sigma_{A}\right)$, given by the functions $\left\{\delta_{x}\right\}_{x \in \Sigma_{A}}$ such that $\delta_{x}(y)=0$ if $y=x$ and $\delta_{x}(y)=1$ if $x=y, x, y \in \Sigma_{A}$. We can consider $\mathcal{B}\left(\ell^{2}\left(\Sigma_{A}\right)\right)$, the space of bounded linear operators on $\ell^{2}\left(\Sigma_{A}\right)$. Now we define the following operators, for $i=1, \ldots, n$,

$$
V_{i} \delta_{x}= \begin{cases}\delta_{i x} & \text { if } \quad A\left(i, x_{1}\right)=1 \\ 0 & \text { otherwise }\end{cases}
$$

The adjoint operator can be shown to be

$$
V_{i}^{*} \delta_{x}= \begin{cases}\delta_{\sigma(x)} & \text { if } x_{1}=i \\ 0 & \text { otherwise }\end{cases}
$$

The Cuntz-Krieger relations are given by

$$
\begin{array}{r}
S_{i}^{*} S_{i}=\left(S_{i}^{*} S_{i}\right)^{2} \quad \forall i \in\{1, \ldots, n\} \\
\sum_{i=1}^{n} S_{i} S_{i}^{*}=1 \\
S_{i}^{*} S_{i}=\sum_{j=1}^{n} A(i, j) S_{j} S_{j}^{*}
\end{array}
$$

It is not difficult to prove that the $V_{i}$ we defined above satisfies the those relations. For example, the second relation comes from the fact that $V_{i} V_{i}^{*} \delta_{x}=\delta_{x}$ if $x_{1}=i$ and zero otherwise, then given any $x \in \Sigma_{A}$, suppose $x_{1}=j$, then $V_{j} V_{j}^{*} \delta_{x}=\delta_{x}$, but $V_{i} V_{i}^{*} \delta_{x}=0$ for all $i \neq j$. Hence $\sum_{i} V_{i} V_{i}^{*}=1$.

The $C^{*}$-Algebra generated by those $V_{i}$, which is a sub- $C^{*}$-algebra of $\mathcal{B}\left(\ell^{2}\left(\Sigma_{A}\right)\right)$ is called the 
Cuntz-Krieger algebra $\mathcal{O}_{A}$. In fact, if we had a set of generators $\left\{Y_{i}\right\}_{i=1}^{n}$ that satisfies the above relations, the universal $C^{*}$-algebra generated by them is isomorphic to $\mathcal{O}_{A}$, a result provided by Cuntz and Krieger in [CK80]. Let us see now how is the set $C\left(\Sigma_{A}\right)$ of complex valued continuous functions relates to $\mathcal{O}_{A}$.

Proposition 25. The $C^{*}$-Algebra $C\left(\Sigma_{A}\right)$ is isomorphic to a $C^{*}$-subalgebra of $\mathcal{O}_{A}$

Proof. For every $f \in C\left(\Sigma_{A}\right)$ consider the map $f \mapsto M_{f}$, where $M_{f}: \ell^{2}\left(\Sigma_{A}\right) \rightarrow \ell^{2}\left(\Sigma_{A}\right)$ is defined as $M_{f} \delta_{x}=f(x) \delta_{x}$. Since $M_{f}$ is an element of $\mathcal{B}\left(\ell^{2}\left(\Sigma_{A}\right)\right)$ we define the set $B:=\left\{M_{f}: f \in C\left(\Sigma_{A}\right)\right\} \subset$ $\mathcal{B}\left(\ell^{2}\left(\Sigma_{A}\right)\right)$. The map $f \mapsto M_{f}$ is a injective $*$-homomorphism from $C\left(\Sigma_{A}\right)$ into $\mathcal{B}\left(\ell^{2}\left(\Sigma_{A}\right)\right)$, so it is, in particular, an isometry and the image of such map is $B$. Next, for a finite word $\alpha$ of length $m$ in the alphabet $\{1, \ldots, n\}$, we define $S_{\alpha}:=S_{\alpha_{1}} S_{\alpha_{2}} \ldots S_{\alpha_{m}}$. If $\mathbb{1}_{[\alpha]}$ is the characteristic function of the cylinder set $[\alpha]$, then, since $[\alpha]$ is clopen $\mathbb{1}_{[\alpha]} \in C\left(\Sigma_{A}\right)$. Using the definitions above, we can verify that $M_{\mathbb{1}_{[\alpha]}}=S_{\alpha} S_{\alpha}^{*} \in \mathcal{O}_{A}$. We conclude that $B \subset \mathcal{O}_{A}$ using the Stone-Weierstrass Theorem.

Consider now $X=\Sigma_{A}$ and the Renault-Deaconu Groupoid for this particular $X$ by

$$
\Gamma_{\sigma}=\left\{(x, k, y) \in X \times \mathbb{Z} \times X: \exists n, m \in \mathbb{N}, k=n-m, \sigma^{n}(x)=\sigma^{m}(y)\right\}
$$

where $\sigma$ is the shift map on $X$. Denote $\mathcal{L}$ to be the set of all admissible and finite words of $X$ and let $|\cdot|: \mathcal{L} \rightarrow \mathbb{N}$ to be the length map. In fact every element of $\Gamma_{\sigma}$ is of the form $(a x,|a|-|b|, b x)$ $a, b \in \mathcal{L}$ and $A\left(a_{|a|}, x_{1}\right)=A\left(b_{|b|}, x_{1}\right)=1$. Consider now the sets:

$$
Z(a, b)=\left\{(a x, l(a)-l(b), b x): x \in \sigma^{|a|}[a] \cap \sigma^{|b|}[b]\right\}
$$

Those sets form a base for the topology on $\Gamma_{\sigma}$ and they are compact. Define as well $\left.Y_{k}:=\{(k x, 1, x): x \in \sigma[k]\}\right)$, for $k=1,2 \ldots, n$. $Y_{k}$ is compact because considering $h_{k}: \sigma[k] \rightarrow \Gamma_{\sigma}$ by $h(x)=(k x, 1, x)$. It is a bijection onto $Y_{k}$ and it is continuous. Continuity is because the only elements of the topology basis that has non empty intersection with $Y_{k}$ are of the form $Z(k a, a)$, it is not difficult to see that $h_{k}^{-1}(Z(k a, a))=[a]$ which is open. $Y_{k}$ is compact is clear by the fact that $[k]$ is compact.

We remember as well some facts about the convolution formula on $C_{c}(G)$ that we saw throughout this text.

Lemma 8. Suppose that $G$ is a locally compact Hausdorff étale groupoid. If $U, V \subseteq G$ are open bisections and $f, g \in C_{c}(G)$ satisfy $\operatorname{supp}(f) \subseteq U$ and $\operatorname{supp}(g) \subseteq V$, then $\operatorname{supp}(f \cdot g) \subseteq U V$ and for $\gamma=\alpha \beta \in U V$, we have

$$
(f \cdot g)(\gamma)=f(\alpha) g(\beta) .
$$

For $f \in C_{c}(G)$ and $h \in C_{c}\left(G^{(0)}\right)$, we have

$$
(h \cdot f)(\gamma)=h(r(\gamma)) f(\gamma) \text { and }(f \cdot h)(\gamma)=f(\gamma) h(s(\gamma))
$$

Theorem 10. Let $S_{k}:=\mathbb{1}_{Y_{k}}$ for $k=1, \ldots, n$. Then $S_{k}$ satisfies the Cuntz-Krieger relations (3.9) and $S_{k}$ generates the $C^{*}$-algebra $C^{*}\left(\Gamma_{\sigma}\right)$.

Proof. It is clear that $S_{k} \in C_{c}\left(\Gamma_{\sigma}\right)$. Observe that:

$$
\mathbb{1}_{Y_{k}}^{*}(\gamma)=\overline{\mathbb{1}_{Y_{k}}\left(\gamma^{-1}\right)}=\mathbb{1}_{Y_{k}^{-1}}(\gamma)
$$

$Y_{k}$ are open bisections, so by the lemma $\operatorname{supp}\left(S_{k}^{*} S_{k}\right)=Y_{k}^{-1} Y_{k}=\sigma[k]$. If $\gamma \in \sigma[k]$ again by the lemma there are $\alpha \in Y_{k}^{-1}$ and $\beta \in Y_{k}$ such that

$$
\mathbb{1}_{Y_{k}^{-1}} \cdot \mathbb{1}_{Y_{k}}(\gamma)=\mathbb{1}_{Y_{k}^{-1}}(\alpha) \mathbb{1}_{Y_{k}}(\beta)=1
$$

In other words 


$$
\mathbb{1}_{Y_{k}^{-1}} \cdot \mathbb{1}_{Y_{k}}=\mathbb{1}_{\sigma[k]}
$$

Observe now that $Y_{k} Y_{k}^{-1}=Z(k, k)$. We have $\mathbb{1}_{Y_{k}} \cdot \mathbb{1}_{Y_{k}^{-1}}=\mathbb{1}_{Z(k, k)}$.

$$
\begin{gathered}
\sum_{j=1}^{n} A(i, j) \mathbb{1}_{\Sigma_{j}} \cdot \mathbb{1}_{\Sigma_{j}^{-1}}=\sum_{j=1}^{n} A(i, j) \mathbb{1}_{\sigma[j]}=\mathbb{1}_{\sigma[k]} \\
\sum_{k=1}^{n} \mathbb{1}_{Y_{k}} \cdot \mathbb{1}_{Y_{k}^{-1}}=\sum_{k=1}^{n} \mathbb{1}_{Z(k, k)}=\mathbb{1}_{\Gamma_{\sigma}^{(0)}}
\end{gathered}
$$

And so the $S_{k}$ indeed satisfies the Cuntz-Krieger relations.

Now consider $\mathcal{P}$ to be the set of all polynomials on the variables $\mathbb{1}_{Y_{k}}$ and $\mathbb{1}_{Y_{k}^{-1}}$. $\mathcal{P}$ forms a *-algebra and we remember that the norm of $C^{*}\left(\Gamma_{\sigma}\right)$ on $C_{c}\left(\Gamma_{\sigma}^{(0)}\right)$ is just the sup norm. We claim the following thing:

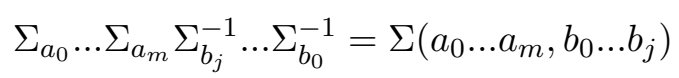

When this makes sense $\left(a_{0} \ldots a_{m}\right.$ and $b_{0} \ldots b_{j}$ are admissible words). I will only show the key steps. Let's see what is $\Sigma_{a_{0}} \Sigma_{a_{1}}$, its elements are of the form $\left(a_{0} x, 1, x\right)\left(a_{1} y, 1, y\right)$, we need then that $x$ starts with $a_{1}$ and $x_{i}=y_{i+1}$, so $\left(a_{0} a_{1} y, 1, a_{1} y\right)\left(a_{1} y, 1, y\right)=\left(a_{0} a_{1} y, 2, y\right) y \in \sigma\left[a_{1}\right]$. It is clear then that elements of $\Sigma_{a_{0}} \ldots \Sigma_{a_{m}}$ are of the form $\left(a_{0} \ldots a_{m} x, m+1, x\right)$ for $x \in \sigma\left[a_{m}\right]$. Now, consider $\Sigma_{a_{0}} \ldots \Sigma_{a_{m}} \sigma_{b_{j}}^{-1}$, its elements are of the form $\left(a_{0} \ldots a_{m} x, m+1, x\right)\left(y,-1, b_{j} y\right)$, so $x=y, x \in \sigma\left[a_{m}\right] \cap \sigma\left[b_{j}\right]$ and it becomes $\left(a_{0} \ldots a_{m} x, m+1-1, b_{j} x\right)$, for the last step, for us, we consider now $\Sigma_{a_{0}} \ldots \Sigma_{a_{m}} \Sigma_{b_{j}}^{-1} \Sigma_{b_{j-1}}^{-1}$, which has elements of the form $\left(a_{0} \ldots a_{m} x, m+1-1, b_{j} x\right)\left(y,-1, b_{j-1} y\right)$, so $y=b_{j} x$ and we have $\left(a_{0} \ldots a_{m}, m+1-2, b_{j-1} b_{j} x\right) x \in \sigma\left[a_{m}\right] \cap \sigma\left[b_{j}\right]$. It is now easy to conclude what we claimed.

In the particular case that $j=m$ and each $a_{i}=b_{i}$, one can show that with the previous identity that

$$
\mathbb{1}_{\Sigma_{a_{0}}} \cdot \ldots \cdot \mathbb{1}_{\Sigma_{a_{m}}} \cdot \mathbb{1}_{\Sigma_{a_{m}}^{-1}}^{-1} \cdot \ldots \cdot \mathbb{1}_{\Sigma_{a_{0}}}^{-1}=\mathbb{1}_{\Sigma\left(a_{0} \ldots a_{m}, a_{0} \ldots a_{m}\right)}
$$

The later can be identified with $\mathbb{1}_{\left[a_{0} \ldots a_{m}\right]}$, where [.] denote the cylinder set on $X$. This means that in $\mathcal{P}$ there is a sub ${ }^{*}$-algebra $B$ which is the polynomials on the variables $\mathbb{1}_{[k]}, k=1, \ldots, n$. One can verify that $\mathbb{1}_{[w]}$ is in $B$ for every admissible word $w$ and so we can conclude that $B$ separates points of $X$. By Stone-Weierstrass theorem $\bar{B}^{\|\cdot\|_{\infty}}=C(X)$, and so $C(X) \subset \overline{\mathcal{P}}^{\|\cdot\|_{C^{*}\left(\Gamma_{\sigma}\right)}} \subseteq C^{*}\left(\Gamma_{\sigma}\right)$. We finally show that $C_{c}\left(\Gamma_{\sigma}\right) \subset \overline{\mathcal{P}}^{\|\cdot\|_{C^{*}\left(\Gamma_{\sigma}\right)}}$ and we conclude that $\overline{\mathcal{P}}^{\|\cdot\|_{C^{*}\left(\Gamma_{\sigma}\right)}}=C^{*}\left(\Gamma_{\sigma}\right)$. Let $f \in C_{c}\left(\Gamma_{\sigma}\right)$. $Z(a, b)$ are open bisections, they are compact and they cover $\Gamma_{\sigma}$. Then as $f$ has compact support, finitely many sets of the form $Z(a, b)$ cover the support of $f$, call them by $\left\{U_{i}\right\}_{i=1}^{m}$. Now we shall be disjointing those $U_{i}$ like this:

Let $W_{1}=U_{1}$ and $W_{i}=U_{i} \backslash \cup_{j=1}^{i-1} U_{j}$. Clearly $\left\{W_{i}\right\}_{i=1}^{m}$ still cover the support of $f$ and observe, since each $U_{j}$ is compact that $W_{i}$ is an open subset of $U_{i}$ and therefore each $W_{i}$ is an open bisection, but pair-wise disjoint. Clearly $f$ can be written as a finite sum $f=\sum_{i=1}^{m} f \mathbb{1}_{W_{i}}$. Take one such $W_{i}$, now consider $g=\left.f \circ r\right|_{W_{i}} ^{-1} \cdot g \in C(X)$ and for $\gamma \in W_{i}$ we have $g(r(\gamma))=f(\gamma)$. Now by the lemma (8) $g * \mathbb{1}_{W_{i}}=f \mathbb{1}_{W_{i}}$.

Now observe that $\mathbb{1}_{W_{i}} \in \mathcal{P}$. Indeed

$$
\mathbb{1}_{W_{i}}=\mathbb{1}_{U_{i} \backslash \cup_{j=1}^{i-1} U_{j}}=\mathbb{1}_{U_{i}} \prod_{j=1}^{i-1}\left(1-\mathbb{1}_{U_{i}}\right)
$$

so $\mathbb{1}_{W_{i}}$ is a polynomial on $\mathbb{1}_{U_{j}}$ and the later is a polynomial on $S_{k}$, in other words $\mathbb{1}_{W_{i}} \in \mathcal{P} \subset$ $\overline{\mathcal{P}}^{\|\cdot\|_{C^{*}\left(\Gamma_{\sigma}\right)}}$.

Since $g \in C(X) \subset \overline{\mathcal{P}}^{\|\cdot\|_{C^{*}\left(\Gamma_{\sigma}\right)}}$ we have $f=\sum_{i=1}^{m} f \mathbb{1}_{W_{i}}=\sum_{i=1}^{m} g * \mathbb{1}_{W_{i}} \in \overline{\mathcal{P}}^{\|\cdot\|_{C^{*}\left(\Gamma_{\sigma}\right)}}$. The proof 
is complete since we conclude that:

$$
C^{*}\left(\Gamma_{\sigma}\right)=\overline{C_{c}\left(\Gamma_{\sigma}\right)}{ }^{\|\cdot\|_{C^{*}\left(\Gamma_{\sigma}\right)}} \subseteq \overline{\mathcal{P}}^{\|\cdot\|_{C^{*}\left(\Gamma_{\sigma}\right)}} \subseteq C^{*}\left(\Gamma_{\sigma}\right)
$$

The Cuntz-Krieger algebras are closely related to the Renault-Deaconu Groupoid. The full or reduced $C^{*}$-Algebra of the Renault-Deaconu Groupoid(they can be shown to be isomorphic), when $X$ is the shift space $X$ we defined above, with finite alphabet and $A$ its transition matrix, then $C^{*}\left(\Gamma_{\sigma}\right)$ is isomorphic to the Cuntz-Krieger Algebra $\mathcal{O}_{A}$ and the isomorphism is given by a map $\rho: \mathcal{O}_{A} \rightarrow C^{*}\left(\Gamma_{\sigma}\right)$ such that $\rho\left(V_{i}\right)=\mathbb{1}_{Y_{k}}$. The proof is non trivial and can be found, in a even more general setting in [KPR98]. What we did above is just one step of such proof. 



\section{Chapter 4}

\section{Phase Transition and Main Result}

This chapter follows close the section 3 of our main reference [Tho17]. The arguments are done in detail, except the proofs of the theorems due S. Neshveyev in [Nes13], which are important but we don't touch here. We use the objects described on the previous sections.

For a Locally compact Hausdorff second-countable space $X$ we can consider the RenaultDeaconu Groupoid $\mathcal{G}$, as already defined. Let $F: X \rightarrow \mathbb{R}$ be a continuous function and the continuous homomorphism $c_{F}: \mathcal{G} \rightarrow \mathbb{R}$ given by

$$
c_{F}(x, k, y)=\sum_{i=0}^{n-1} F\left(\sigma^{i}(x)\right)-\sum_{i=0}^{m-1} F\left(\sigma^{i}(y)\right)
$$

when $(x, k, y) \in \mathcal{U}_{W, V}^{n, m}$. Also, the one-parameter group $\tau^{F}$ on $C^{*}(\mathcal{G})$ such that

$$
\tau_{t}^{F}(f)(x, k, y)=e^{i t c_{F}(x, k, y)} f(x, k, y)
$$

for $f \in C_{c}(\mathcal{G})$. We already know one kind of $K M S$ state, indeed, a main concern in chapter 4 was to prove that given a $e^{\beta F_{-}}$-conformal measure $m$ there is associated to it a $K M S_{\beta}$ state $\phi_{m}$ given by

$$
\phi_{m}(f)=\int_{X} f(x, 0, x) d m(x)
$$

for all $f \in C_{c}(\mathcal{G})$.

One question that arises is that if there are others $K M S_{\beta}$ states. The answer is affirmative as we will see below.

Let $x \in X$ be a $\sigma$-periodic point, i.e there is $p \in \mathbb{N}$ such that $\sigma^{p}(x)=x$. We take $p$ as the minimal period of $x$. Let this $x$ be such that

$$
\sum_{j=0}^{p-1} F\left(\sigma^{j}(x)\right)=0
$$

Assume that $\beta \in \mathbb{R}$ satisfies the following condition

$$
M=\sum_{n=1}^{\infty} \sum_{y \in Y_{n}} \exp \left(-\beta \sum_{j=0}^{n-1} F\left(\sigma^{j}(y)\right)\right)<\infty
$$

Where

$$
Y_{n}=\sigma^{-n}(x) \backslash \bigcup_{j=0}^{n-1} \sigma^{-j}(x) .
$$

In those conditions we define a measure $m_{x}$ as 


$$
m_{x}=(1+M)^{-1}\left(\delta_{x}+\sum_{n=1}^{\infty} \sum_{y \in Y_{n}} \exp \left(-\beta \sum_{j=0}^{n-1} F\left(\sigma^{j}(y)\right)\right) \delta_{y}\right)
$$

is a Borel probability measure and it is $e^{\beta F}$-conformal.

Lemma 9. Let $\lambda \in \mathbb{T}$, the complex circle, and $y \in \cup_{n=0}^{\infty} \sigma^{-n}(x)$, the map $(y, k p, y) \mapsto \lambda^{k}$ is a character of the group $\mathcal{G}_{y}^{y}$.

Proof. we remember that $\mathcal{G}_{y}^{y}$ is the set $r^{-1}(\{y\}) \cap s^{-1}(\{y\})$ and his elements then are of the form $(y, l, y) \in \mathcal{G}$. This means that there is $n, m \in \mathbb{N}$ such that $\sigma^{n}(y)=\sigma^{m}(y), n-m=l$ and more than that $y=\sigma^{k}(x)$ for some $k \in \mathbb{N}$. Without loss of generality, suppose $n \geq m$, then $\sigma^{n+k}(x)=\sigma^{m+k}(x)$ implies $\sigma^{l}(x)=x$, hence $l$ is a multiple of the period $p$, if $m \geq n$ we would have $l$ to be a negative multiple of $p$. The function is then well defined and it is easy to verify that it is a homomorphism of the Group $\mathcal{G}_{y}^{y}$ into $\mathbb{T}$.

Now, theorems 1.1 and 1.3 of [Nes13], tell us that there exists a $K M S_{\beta}$ state $\phi_{x}^{\lambda}$ such that

$$
\phi_{x}^{\lambda}(f)=\int_{X} \sum_{k \in \mathbb{Z}} \lambda^{k} f(y, k p, y) d m_{x}(y)
$$

for all $f \in C_{c}(\mathcal{G})$. We remember that $x$ is a periodic point in which condition (4.1) applies.

Definition 29. For $z \in X$, we denote by $\mathcal{O}(z)$ the full orbit of $z$, i.e, the set of points $y \in X$ such that

$$
\sigma^{n}(z)=\sigma^{m}(y)
$$

for some $n, m \in \mathbb{N}$

A point $z \in X$ is called aperiodic when $\mathcal{O}(z)$ does not contain a periodic orbit, i.e, there is no $y \in \mathcal{O}(z)$ and $p \in \mathbb{N}$ such that $\sigma^{p}(y)=y$.

Remark 13. If there were such a periodic orbit, then $\sigma^{n}(z)=\sigma^{m}(y)=\sigma^{m}\left(\sigma^{k p}(y)\right)=\sigma^{m+k p}(y)$ for every $k \in \mathbb{N}$. We could make $k$ big enough so that $m+k p>n$ and we would have $\sigma^{m+k p-n}(z)=z$, which shows that $z$ is periodic.

For such a point $z$ aperiodic, we can define $\mathcal{F}: \mathcal{O}(z) \rightarrow \mathbb{R}$ such that

$$
\mathcal{F}(y)=\sum_{j=0}^{m-1} F\left(\sigma^{j}(y)\right)-\sum_{j=0}^{n-1} F\left(\sigma^{j}(z)\right)
$$

where $n, m$ are the numbers associated with $y$ in equation (4.3).

Definition 30. Let $\beta \in \mathbb{R}$. We say that $z$ is $\beta$-summable when

$$
M=\sum_{y \in \mathcal{O}(z)} e^{-\beta \mathcal{F}(y)}<\infty .
$$

Proposition 26. Let $z \in X$ be aperiodic. The Borel probability measure

$$
m_{z}:=M^{-1} \sum_{y \in \mathcal{O}(z)} e^{-\beta \mathcal{F}(y)} \delta_{y}
$$

is $e^{\beta F}$-conformal.

Proof. The fact that $m_{z}$ is a probability measure is trivial. Now given $A$ measurable such that $\left.\sigma\right|_{A}$ is injective we compute,

$$
m_{z}(\sigma(A))=M^{-1} \sum_{y \in \mathcal{O}(z) \cap \sigma(A)} e^{-\beta \mathcal{F}(y)}
$$


Next we observe that $\mathcal{O}(z) \cap \sigma(A)=\sigma(\mathcal{O}(z) \cap A)$ and $\sigma$ is a bijection from $\sigma(\mathcal{O}(z) \cap A)$ onto $\mathcal{O}(z) \cap \sigma(A)$. Indeed if $y \in \sigma(\mathcal{O}(z) \cap A)$, then there is $x \in A$ s.t $y=\sigma(x)$, and this $x$ is unique. Clearly $y \in \sigma(A)$ and since $\sigma^{m}(y)=\sigma^{n}(z)$ implies $\sigma^{m+1}(x)=\sigma^{n}(z)$ and we have $x \in \mathcal{O}(z)$. This proves that $\sigma(\mathcal{O}(z) \cap A) \subset \mathcal{O}(z) \cap \sigma(A)$. The other side is analogous and because of the uniqueness of the $x$ above, $\sigma$ is a bijection on those sets. Now, using the change of variables $y \rightarrow \sigma(y)$ on equation (4.4), we have,

$$
m_{z}(\sigma(A))=M^{-1} \sum_{y \in \mathcal{O}(z) \cap A} e^{-\beta \mathcal{F}(\sigma(y))}=M^{-1} \sum_{y \in \mathcal{O}(z) \cap A} e^{-\beta\left(\sum_{j=1}^{m} F\left(\sigma^{j}(y)\right)-\sum_{j=0}^{n-1} F\left(\sigma^{j}(z)\right)\right)}
$$

Where $m$ and $n$ are such that $\sigma^{m+1}(y)=\sigma^{n}(z)$. On the other hand, a the expression for $\int_{A} e^{\beta F(y)} d m_{z}(y)$ is

$$
\int_{A} e^{\beta F(y)} d m_{z}(y)=M^{-1} \sum_{y \in \mathcal{O}(z) \cap A} e^{\beta F(y)} e^{-\beta \mathcal{F}(y)}=M^{-1} \sum_{y \in \mathcal{O}(z) \cap A} e^{-\beta\left(\sum_{j=1}^{m-1} F\left(\sigma^{j}(y)\right)-\sum_{j=0}^{n-1} F\left(\sigma^{j}(z)\right)\right)}
$$

where $m$ and $n$ are such that $\sigma^{m}(y)=\sigma^{n}(z)$. With that in mind, these expressions concide and we have that $m_{z}$ is $e^{\beta F}$ conformal.

Remember that we want to explore the connection between conformal measures and $K M S_{\beta}$ states. In fact, under suitable hypotheses, we have a complete characterization of the extremal $K M S_{\beta}$ states as follows:

Theorem 11. Let $\beta \in \mathbb{R} \backslash\{0\}$. Assume that the periodic point of $\sigma$ are countable. The extremal $K M S_{\beta}$ states for $\tau^{F}$ are

1. States $\phi_{m}$, where $m$ is an extremal and continuous(non-atomic) $e^{\beta F}$-conformal borel probability measure on $X$

2. The states $\phi_{x}^{\lambda}$, where $\lambda \in \mathbb{T}$ and $x$ is a p-periodic point for $\sigma$ for which (4.1) and (4.2) both hold, and

3. The states $\phi_{m_{z}}$, where $z$ is aperiodic and $\beta$-summable

Proof. It is as combination of arguments which can be found in [Nes13] and [Tho12].

We will apply what we discussed above in the particular case that $X=\{0,1\}^{\mathbb{N}}$, the full shift, but this time, to make it consistent with the source, we are using the alphabet $\{0,1\}$, the discussions we had in previous chapters when considering the alphabet $\{1,2\}$ and $\Sigma_{2}$ are entirely analogous, just changing $2 \rightarrow 0$. Let us now define our potential function $F:\{0,1\}^{\mathbb{N}} \rightarrow \mathbb{R}$

$$
F\left(\left(x_{i}\right)_{i=1}^{\infty}\right)= \begin{cases}\frac{1}{\min \left\{i: x_{i}=0\right\}}, & \left(x_{i}\right)_{i=1}^{\infty} \neq 1^{\infty} \\ 0 & \left(x_{i}\right)_{i=1}^{\infty}=1^{\infty}\end{cases}
$$

This is clearly influenced by the potential due to Hofbauer, that we defined in the preliminaries. In this case, the sequence of $a_{k}$ is given by $a_{k}=\frac{1}{k}$. Now, we remember that we can see $F$ as an element of $\mathcal{O}_{2}$, due to the function $F \mapsto M_{F}$. Since $F$ is a real value function, $F$ is self adjoint and so if $M_{f}$ in $\mathcal{O}_{2}$. Then we can define the one parameter group of automorphism of $\mathcal{O}_{2}$ as $\eta_{t}^{F}\left(V_{i}\right)=e^{i t M_{F}} V_{i}$, where $V_{i}$ are the operators we previously defined. Now due to the $*$-isomorphism $\rho: \mathcal{O}_{2} \rightarrow C^{*}\left(\Gamma_{\sigma}\right)$, we can observe that $\eta_{t}^{F}$ becomes $\tau_{t}^{F}$ under this isomorphism. Indeed $\eta_{t}^{F}: \mathcal{O}_{2} \rightarrow \mathcal{O}_{2}$, then using $\rho$, we need to show that $\eta_{t}^{F}=\rho^{-1} \circ \tau_{t}^{F} \circ \rho$. In fact

$$
\rho^{-1} \circ \tau_{t}^{F} \circ \rho\left(V_{i}\right)=\rho^{-1} \tau_{t}^{F}\left(\mathbb{1}_{\left\{x, 1, \sigma(x): x_{1}=i\right\}}\right)=\rho^{-1}\left(e^{i t F(\cdot)} \mathbb{1}_{\left\{x, 1, \sigma(x): x_{1}=i\right\}}\right)=e^{i t M_{F}} V_{i}=\eta_{t}^{F}\left(V_{i}\right) .
$$


This basically means that if we know the $K M S_{\beta}$ states for $\tau^{F}$, automatically we know for $\eta^{F}$. Another observation here is that there is only one periodic point $x \in\{0,1\}^{\mathbb{N}}$ such that the condition 4.1 is satisfied, the point $1^{\infty}$.

Let us define the critical for this potential. The main result of this chapter is to show that this point is precisely the value of $\beta$ where the cardinality of the set of $K M S_{\beta}$ changes. Let $\beta_{0} \geq 0$ be such that

$$
\sum_{k=1}^{\infty} \exp \left(-\beta_{0} \sum_{j=1}^{k} \frac{1}{j}\right)=1
$$

Remark 14. Let us prove that such $\beta_{0}$ exists. Define $H_{k}:=\sum_{j=1}^{k} 1 / k$, the harmonic numbers. We use a common lower and upper bound for $H_{n}$, usually deduced in calculus courses. We have

$$
\log (k+1)<H_{k} \leq 1+\log (k)
$$

Then, for $\beta>0$

$$
-\beta \log (k+1)>-\beta H_{k} \geq-\beta(1+\log (k))
$$

Define $g(\beta):=\sum_{k=1}^{\infty} \exp \left(-\beta H_{k}\right)$. Using the inequality (4.7), we deduce

$$
\begin{gathered}
\sum_{k=1}^{\infty} \exp (-\beta-\beta \log (k)) \leq \sum_{k=1}^{\infty} \exp \left(-\beta H_{k}\right)<\sum_{k=1}^{\infty} \exp (-\beta \log (k+1)) \\
\exp (-\beta) \sum_{k=1}^{\infty} k^{-\beta} \leq g(\beta)<\sum_{k=1}^{\infty}(k+1)^{-\beta}
\end{gathered}
$$

Note that $g(\beta)$ converges only if $\beta>1$. Now, one can verify that for $\beta=8$ we get $g(\beta)<1$ and for $\beta=1.2 g(\beta)>1$. The function $g$ is continuous on $(1,+\infty)$, since, for $\beta_{n} \rightarrow \beta$, we can exchange the order of the infinite sum with the limit by monotonous convergence theorem. Then, we conclude there exists a $\beta_{0} \in \mathbb{R}$ such that $g\left(\beta_{0}\right)=1$.

Now, we need two technical lemmas and a standard proposition about the set of KMS states be closed to get the main result about the phase transition to this potential:

Lemma 10. For each $\beta \in \mathbb{R}$ there is at most one $e^{\beta F}$-conformal probability measure for $\sigma$, and none if $\beta<\beta_{0}$

Proof. Let $u$ be a word, i.e $u \in\{0,1\}^{n}$ and let $[u]$ correspond to its cylinder set. A $e^{\beta F}-$ conformal borel probability measure $\mu$ must satisfy, using $A=[0]$

$$
\mu(\sigma([0]))=\int_{[0]} e^{\beta F(x)} d \mu(x)=\int_{[0]} e^{\beta} d \mu(x)=e^{\beta} \mu([0])
$$

Remark 15. The above choice for A makes sense to the definition because the shift $\sigma$ is one-to-one when we restrict its domain on the cylinder set [0], in fact this is true for every cylinder set in the form $[0 w]$ or $[1 w]$ where $w$ is a word .

Note that $\mu(\sigma([0]))=\mu\left(\{0,1\}^{\mathbb{N}}\right)=1$, due to be a probability measure. We have

$$
e^{\beta} \mu([0])=1
$$

So we have $\mu([0])=e^{-\beta}$ and $1-e^{-\beta}=1-\mu([0])=\mu(\Omega \backslash[0])=\mu([1])$. It means that every $e^{\beta F}-$ conformal borel probability measure must satisfy these values at those cylinders, in fact we show that this happens to every cylinder. Assume that we have determined $\mu([w])$ for every word $w \in\{0,1\}^{n}$, we will be showing the uniqueness by induction. Use in the definition of $e^{\beta F}$-conformal measure $A=[0 w]$, so that 


$$
\mu(\sigma([0 w]))=\mu([w])=\int_{[0 w]} e^{\beta F(x)} d \mu(x)=e^{\beta} \mu([0 w])
$$

Just to make it clear, $F(x)=1$ in the above integral because we are integrating in the set $[0 w]$ and for all $x \in[0 w]$ we have $F(x)=1$, by the definition. We conclude that $\mu([0 w])=e^{-\beta} \mu([w])$. Lets assume that $w$ is a word that is not composes only by 1 's, say $j$ is the position of the first 0 . We have, choosing $A=[1 w]$

$$
\mu(\sigma([1 w]))=\mu([w])=\int_{[1 w]} e^{\beta F(x)} d \mu(x)=e^{\frac{\beta}{j+1}} \mu([1 w])
$$

the third equality is because the first zero in $[1 w]$ is on $j+1$. We conclude $\mu([1 w])=e^{-\frac{\beta}{j+1}} \mu([w])$. We have, then, determined the value of $\mu([u])$ for every word $u \in\{0,1\}^{n+1}$, except the word composed only on 1's. Lets say the word composing only on 1's is $h \in\{0,1\}^{n+1}$, we can't determine his measure with the process above, but it's measure depends on the measures of the others words $u \in\{0,1\}^{n+1}$, because its true that

$$
\bigcup_{u \in\{0,1\}^{n+1} \backslash h}[u] \cup[h]=\{0,1\}^{\mathbb{N}}
$$

This union is disjoint, so

$$
\mu([h])=1-\sum_{u \in\{0,1\}^{n+1} \backslash h} \mu([u])
$$

This proves that every $e^{\beta F}$-conformal probability measure must have a certain value at the cylinder. It so happens that the cylinders generate the borel $\sigma$-algebra, so we by the Carathéodory extension theorem for a measure, we have at most one $e^{\beta F}$-conformal probability measure for $\sigma$. Now we need to prove that there are none if $\beta<\beta_{0}$. Consider the sets [0], [10], [110], [1110], they are mutually disjoint. We have that for every Borel measure $\mu$ satisfying the definition of $e^{\beta F}$-conformal measure that

$$
\mu\left(\left[1^{k-1} 0\right]\right)=\exp \left(-\beta \sum_{j=1}^{k} \frac{1}{j}\right)
$$

We prove this by induction in $k$. If $k=1$ it is obviously satisfied. Let it be true for $k$ then we have

$$
\left.\mu\left(\left[1^{k} 0\right]\right)=\mu\left(\left[11^{k-1} 0\right)\right]\right)=e^{\frac{-\beta}{k+1}} \mu\left(\left[1^{k-1} 0\right]\right)=e^{\frac{-\beta}{k+1}} \exp \left(-\beta \sum_{j=1}^{k} \frac{1}{j}\right)=\exp \left(-\beta \sum_{j=1}^{k+1} \frac{1}{j}\right)
$$

so it is proven the identity, now since they are mutually disjoint when we sum for all $k \in \mathbb{N}$ we must have that $\sum_{k} \mu\left(\left[1^{k-1} 0\right]\right) \leq 1$, so

$$
\sum_{k} \exp \left(-\beta \sum_{j=1}^{k} \frac{1}{j}\right) \leq 1
$$

i.e $\beta \geq \beta_{0}$. The lemma is proved.

Lemma 11. Let $\beta \in \mathbb{R}$. Then

$$
\sum_{n=1}^{\infty} \sum_{x \in \sigma^{-n}\left(1^{\infty}\right) \backslash \sigma^{-n+1}\left(1^{\infty}\right)} \exp \left(-\beta \sum_{j=0}^{n-1} F\left(\sigma^{j}(x)\right)\right)<\infty
$$

iff $\beta>\beta_{0}$ 
Proof. We set $Y_{0}=\left\{1^{\infty}\right\}$ and $Y_{n}=\sigma^{-n}\left(1^{\infty}\right) \backslash \sigma^{-n+1}\left(1^{\infty}\right), n \geq 1$.Then

$$
Y_{n}=0 Y_{n-1} \cup 10 Y_{n-2} \cup 110 Y_{n-3} \cup 1110 Y_{n-4} \cup \cdots \cup 1^{n-1} 0 Y_{0}
$$

This is seen by the following: $Y_{n}$ must be of the form $\left(x_{1}, \ldots, x_{n-1}, 0,1, \ldots\right)$, where those $x_{i}$ may be 0 or 1 . We see that

$$
\left(x_{1}, \ldots, x_{n-1}, 0,1, \ldots\right)=\left(0, x_{2}, \ldots, x_{n-1}, 0,1, \ldots\right) \cup\left(1, x_{2}, \ldots, x_{n-1}, 0,1, \ldots\right)
$$

But the first term is precisely the form of $0 Y_{n-1}$. Now is matter of induction, since we can do the same for $\left(1, x_{2}, \ldots, x_{n-1}, 0,1, \ldots\right)$. As a illustration we shall do one more step.

$$
\left(1, x_{2}, \ldots, x_{n-1}, 0,1, \ldots\right)=\left(1,0, x_{3}, \ldots, x_{n-1}, 0,1, \ldots\right) \cup\left(1,1, x_{3}, \ldots, x_{n-1}, 0,1, \ldots\right)
$$

The first term is precisely of the form of $10 Y_{n-2}$, the process goes on analysing $\left(1,1, x_{3}, \ldots, x_{n-1}, 0,1, \ldots\right)$. Now, let

$$
Z_{n}=\sum_{x \in Y_{n}} \exp \left(-\beta \sum_{j=0}^{n} F\left(\sigma^{j}(x)\right)\right)
$$

for $n \geq 0$, and $s_{k}=1+1 / 2+\cdots+1 / k$. It follows from the equation for $Y_{n}$ that

$$
Z_{n}=e^{-\beta s_{1}} Z_{n-1}+e^{-\beta s_{2}} Z_{n-2}+\cdots+e^{-\beta s_{n}} Z_{0}
$$

for all $n \geq 1$. For that let us analyse some particular elements. The union in (4.8) are disjoint, so the summation on $Y_{n}$ is the same as the summation over $0 Y_{n}, 10 Y_{n-2}$, so on. Let us see how we compute $\sum_{x \in 0 Y_{n-1}} \exp \left(-\beta \sum_{j=0}^{n} F\left(\sigma^{j}(x)\right)\right)$, the others will be somewhat analogous. Observe that

$$
\begin{aligned}
\sum_{x \in 0 Y_{n-1}} \exp \left(-\beta \sum_{j=0}^{n} F\left(\sigma^{j}(x)\right)\right) & =\sum_{x \in 0 Y_{n-1}} \exp \left(-\beta \sum_{j=1}^{n} F\left(\sigma^{j}(x)-\beta\right)\right) \\
& =\sum_{x \in 0 Y_{n-1}} \exp \left(-\beta \sum_{j^{\prime}=0}^{n-1} F\left(\sigma^{j^{\prime}+1}(x)-\beta s_{1}\right)\right) \\
& =\sum_{x \in Y_{n-1}} \exp \left(-\beta \sum_{j^{\prime}=0}^{n-1} F\left(\sigma^{j^{\prime}}(x)\right)\right) e^{-\beta s_{1}} \\
& =Z_{n-1} e^{-\beta s_{1}}
\end{aligned}
$$

Now we use (4.9) to conclude that

$$
\sum_{n=1}^{N} Z_{n} \leq\left(\sum_{n=0}^{N} Z_{n}\right)\left(\sum_{k=1}^{N} e^{-\beta s_{k}}\right) \leq \sum_{n=1}^{2 N} Z_{n}
$$

for $N \geq 1$. We deduce from the above that $\sum_{k=1}^{\infty} e^{-\beta s_{k}}<1$ iff $\sum_{n=1}^{\infty} Z_{n}<\infty$. We conclude the lemma since the condition $\sum_{k=1}^{\infty} e^{-\beta s_{k}}<1$ is the same as saying $\beta>\beta_{0}$

Proposition 27. Let $\mathfrak{A}$ be a $C^{*}$-algebra with identity 1 , and $\left\{\tau^{n}\right\}_{n \geq 1}$ a sequence of strongly continuous one-parameter groups of $*$-automorphisms of $\mathfrak{A}$ converging strongly to a one-parameter group $\tau$, i.e,

$$
\lim _{n \rightarrow \infty}\left\|\tau_{t}^{n}(A)-\tau_{t}(A)\right\|=0
$$

for each $t \in \mathbb{R}$ and $A \in \mathfrak{A}$. Assume that there exists a $\left(\tau_{t}^{n}, \beta_{n}\right)-K M S$ state $\omega_{n}$ on $\mathfrak{A}$ for each $n$, 
where $\left\{\beta_{n}\right\}_{n \geq 1} \subset \mathbb{R} \cup \pm \infty$ converges to a $\beta \in \mathbb{R} \cup\{ \pm \infty\}$, i.e,

$$
\lim _{n \rightarrow \infty} \beta_{n}=\beta .
$$

It follows that each weak ${ }^{*}$-limit point $\omega$ of the sequence $\left\{\omega_{n}\right\}$ is a $(\tau, \beta)$-KMS state on $\mathfrak{A}$. In particular there exists a $(\tau, \beta)-K M S$ state on $\mathfrak{A}$

Proof. See [BR81]

Corollary 2. Let $\mathfrak{A}$ be a $C^{*}$-algebra with identity 1 , and $\tau$ a strongly continuous one-parameter group of $*$-automorphisms of $\mathfrak{A}$. Then the set $\mathfrak{C}:=\{\beta \in \mathbb{R} \mid \exists a(\tau, \beta)$-KMS state $\}$ is closed.

Proof. Let $\beta_{n}$ be a sequence in $\mathfrak{C}$ converging to $\beta$. Then there exists a sequence $\omega_{n}$ of $\left(\tau, \beta_{n}\right)-K M S$ states. By Proposition 27 there exists a $(\tau, \beta)$-KMS state $\omega$, which means that $\beta \in \mathfrak{C}$.

We can now prove the main theorem from this chapter which says that there is a phase transition for the Hofbauer potential also in the quantum case. The result below is a simplified version of the original one from [Tho17], where the author also obtained a description for the set of $K M S_{\beta}$ states when $\beta>\beta_{0}$.

Theorem 12. Let $\eta^{F}$ be the one-parameter group of automorphism on $\mathcal{O}_{2}$ defined in this section. Then let $\beta_{0} \geq 0$ be such that

$$
\sum_{k=1}^{\infty} \exp \left(-\beta_{0} \sum_{j=1}^{k} \frac{1}{j}\right)=1
$$

There is no $K M S_{\beta}$ states for $\eta^{F}$ when $\beta<\beta_{0}$, a unique $K M S_{\beta_{0}}$ state and for $\beta>\beta_{0}$ uncountable many extremal $K M S_{\beta}$ states.

Proof. The Theorem comes from the last two Lemmas and Theorem (11).For $\beta<\beta_{0}$ Lemma 10 implies that there are no $e^{\beta F_{-}}$-conformal probability measure and by the Theorem 11, none of possibilities are available, so there are no $K M S_{\beta}$ for $\beta<\beta_{0}$. For $\beta>\beta_{0}, x=1^{\infty}$ satisfies (4.1) and by Lemma 11, $\beta$ satisifes condition(4.2). By Theorem 11 item 2 we have infinitely many extremal $K M S_{\beta}$ states, one for each $\lambda \in \mathbb{T}$. This means, for now, that the set of $\beta$ s.t there are $K M S_{\beta}$ states is $\left(\beta_{0},+\infty\right)$, but by Corollary 2 , this set must be closed, then there is a $K M S_{\beta_{0}}$ state. It is unique because by Lemma 11, the KMS states on item 2 in Theorem 11 do not occur and by Lemma 10 there is only one $e^{\beta_{0} F}-$ conformal measure. 



\section{Bibliography}

[ABF87] M. Aizenman, D. J Barsky, and R. Fernández. The phase transition in a general class of ising-type models is sharp. Journal of Statistical Physics, 47(3-4):343-374, 1987. 9

[BCCP15] R. Bissacot, M. Cassandro, L. Cioletti, and E. Presutti. Phase transitions in ferromagnetic ising models with spatially dependent magnetic fields. Communications in Mathematical Physics, 337(1):41-53, 2015. 9

[Bla85] B. Blackadar. Shape theory for c*-algebras. Mathematica Scandinavica, pages 249-275, 1985. 17

[Bov06] A. Bovier. Statistical mechanics of disordered systems: a mathematical perspective, volume 18. Cambridge University Press, 2006. 1

[Bow08] R. Bowen. Equilibrium States and the Ergodic Theory of Anosov Diffeomorphisms (Lecture Notes in Mathematics). Springer, 2008. 1, 2, 5, 8

[BR79] O. Bratteli and DW. Robinson. Operator Algebras and Quantum Statistical Mechanics 1. Springer Verlag, Berlin, 1979. 10

[BR81] O. Bratteli and DW. Robinson. Operator Algebras and Quantum Statistical Mechanics 2. Springer Verlag, Berlin, 1981. 10, 59

[CK80] J. Cuntz and W. Krieger. A class of $c^{*}$-algebras and topological markov chains. Inventiones mathematicae, 56(3):251-268, 1980. 49

[CL17] L. Cioletti and A. O. Lopes. Interactions, specifications, dlr probabilities and the ruelle operator in the one-dimensional lattice. Discrete and Continuous Dynamical Systems A, 37(3):6139, 2017. 1, 8

[Con90] J. B. Conway. A course in functional analysis, volume 96 of graduate texts in mathematics, 1990. 29

[Cun77] J. Cuntz. Simple c*-algebra generated by isometries. Communications in mathematical physics, 57(2):173-185, 1977. 2

[Dav96] Kenneth R Davidson. $C^{*}$-algebras by example, volume 6. American Mathematical Soc., 1996. 10

[Dob68] R. L. Dobrushin. Gibbsian random fields for lattice systems with pairwise interactions. Functional Analysis and its applications, 2(4):292-301, 1968. 2

[DU91] M. Denker and M. Urbański. On the existence of conformal measures. Transactions of the American Mathematical Society, 328(2):563-587, 1991. 37

[FV17] S. Friedli and Y. Velenik. Statistical mechanics of lattice systems: a concrete mathematical introduction. Cambridge University Press, 2017. 1

[Geo11] H.O Georgii. Gibbs measures and phase transitions, volume 9. Walter de Gruyter, 2011. 1,9 
[HHW67] Rudolf Haag, Nicolaas Marinus Hugenholtz, and Marinus Winnink. On the equilibrium states in quantum statistical mechanics. Communications in Mathematical Physics, $5(3): 215-236,1967.2$

[Hof77] F. Hofbauer. Examples for the nonuniqueness of the equilibrium state. Transactions of the American Mathematical Society, 228:223-223, 1977. i, iii, 2, 8, 10

[Isr15] R. B. Israel. Convexity in the theory of lattice gases. Princeton University Press, 2015. 1

[Joh70] R. A. Johnson. Atomic and nonatomic measures. Proceedings of the American Mathematical Society, 25(3):650-655, 1970. 46

[Kea72] M. Keane. Strongly mixingg-measures. Inventiones mathematicae, 16(4):309-324, 1972. 7

[Kec95] A. Kechris. Classical descriptive set theory. Springer Science \& Business Media, 1995. 45

[Kel98] G. Keller. Equilibrium states in ergodic theory, volume 42. Cambridge university press, 1998. 2

[KPR98] A. Kumjian, D. Pask, and I. Raeburn. Cuntz-krieger algebras of directed graphs. Pacific Journal of Mathematics, 184(1):161-174, 1998. 51

[KSS07] M. Kesseböhmer, M. Stadlbauer, and B. Stratmann. Lyapunov spectra for kms states on cuntz-krieger algebras. Mathematische Zeitschrift, 256(4):871-893, 2007. 2

[LR69] OE Lanford and D. Ruelle. Observables at infinity and states with short range correlations in statistical mechanics. Communications in Mathematical Physics, 13(3):194-215, 1969. 2

[Mey13] T. Meyerovitch. Gibbs and equilibrium measures for some families of subshifts. Ergodic Theory and Dynamical Systems, 33(3):934-953, 2013. 2

[Mur14] G. J. Murphy. $C^{*}$-algebras and operator theory. Academic press, 2014. 10

[Nes13] S. Neshveyev. Kms states on the $c^{*}$-algebras of non-principal groupoids. Journal of Operator Theory, 70(2):513-530, 2013. 53, 54, 55

[Phi89] N. C. Phillips. Inverse limits of $\mathrm{c}^{*}$-algebras and applications. In David E. Evans and Masamichi Takesaki, editors, Operator Algebras and Applications: Volume 1, Structure Theory; K-theory, Geometry and Topology (London Mathematical Society Lecture Note Series), pages 127-185. Cambridge University Press, 1989. 17

[PP90] W. Parry and M. Pollicott. Zeta functions and the periodic orbit structure of hyperbolic dynamics. Société mathématique de France, 1990. 1

[Put] I. F. Putnam. Lecture Notes on $C^{*}$-algebras. 21

[Ren80] J. Renault. A groupoid approach to $C^{*}$-algebras, volume 793. Springer, 1980. 2, 25, 29, 39

[Ren09] J. Renault. C*-algebras and Dynamical Systems. IMPA, 2009. 46

[Rue04] D. Ruelle. Thermodynamic formalism: the mathematical structure of equilibrium statistical mechanics. Cambridge University Press, 2004. 1, 2

[Sim] A. Sims. Hausdorff étale groupoids and their c*-algebra. 21, 25 
[Sim14] B. Simon. The statistical mechanics of lattice gases, volume 1. Princeton University Press, 2014. 1

[Tho12] K. Thomsen. Kms states and conformal measures. Communications in Mathematical Physics, pages 1-26, 2012. 55

[Tho14] K. Thomsen. Kms weights on groupoid and graph $\mathrm{c}^{*}$-algebras. Journal of Functional Analysis, 266(5):2959-2988, 2014. 38

[Tho17] K. Thomsen. Phase transition in $\mathcal{O}_{2}$. Communications in Mathematical Physics, 349(2):481-492, 2017. i, iii, 2, 8, 53, 59

[VO16] M. Viana and K. Oliveira. Foundations of ergodic theory, volume 151. Cambridge University Press, 2016. 6, 7

[Wa175] P. Walters. Ruelle's operator theorem and g-measures. Transactions of the American Mathematical Society, 214:375-387, 1975. 7 